\title{
ARL AnNual Salary Survey 2010-2011
}

Compiled and Edited by

Martha KyRILLIDOU

SHANEKA MORRIS

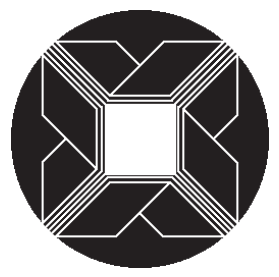

Association of ResEARCh LibraRIES

WASHINGTON, DC 
ARL Annual Salary Survey 2010-2011

The quantitative tables presented in this publication are not indicative of performance and should not be used as measures of library quality. In comparing any individual library to ARL medians or to other ARL members, one must be careful to make such comparisons within the context of differing institutional characteristics.

Custom reports based on the Salary Survey data are also available. Contact the ARL Statistics and Measurement Program Officer for further information.

Visit the ARL Statistics and Measurement Program online at http://www.arl.org/stats/.

Published by the

Association of Research Libraries

Washington, DC 20036

www.arl.org

ISSN 0361-5669

ISBN 1-59407-866-1

EAN 978-1-59407-866-8

(C) 2011

The compilation is copyrighted by the Association of Research Libraries. Blanket permission is granted to reproduce and distribute copies of this work for nonprofit, educational, or library purposes, provided that the author, source, and copyright notice are included on each copy. This permission is in addition to rights of reproduction granted under Sections 107, 108, and other provisions of the US Copyright Act.

The paper used in this publication meets the minimum requirements of the American National Standard for Information Science and National Information Standards Organization standard-Permanence of Paper for Publications and Documents in Libraries and Archives, ANSI/NISO Z39.48-1992(R1997). 


\section{CONTENTS}

\section{SALARY SuRvey Trends 2010-2011}

Figure 1: $\quad$ Ethnicity/Race of Professional Staff in US ARL University Libraries, FY 2010-2011............... 8

Figure 2: $\quad$ Minority Professionals by Region in US ARL University Libraries, FY 2010-2011 .................. 9

Figure 3: Race/Ethnicity and Sex Distribution of Professional Staff in ARL University

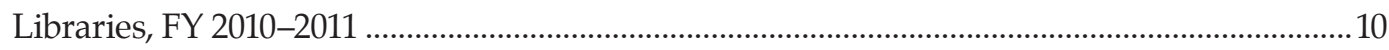

Figure 4: Distribution of Functional Specialist Job Sub-Codes by Type of Library .................................12

Figure 5: Distribution of Functional Specialist Job Sub-Codes' Average Salaries by Sex ........................13

Figure 6: Average Salaries and Average Years of Experience of Library Professionals in Libraries with Three, Four, and Five Step Rank Structures, FY 2010-2011

Salary Levels for Staff in ARL Libraries

Table 1: Distribution by Salary Level .19

Table 2: $\quad$ Salary Trends in ARL University Libraries............................................................................ 20

Table 3: $\quad$ Salary Trends in US ARL University Libraries..............................................................................21

Table 4: $\quad$ Salary Trends in Canadian ARL University Libraries ............................................................... 22

\section{ARL NONUNIVERSITY LIBRARIES}

Table 5: $\quad$ Median and Beginning Professional Salaries in ARL Nonuniversity Libraries ...................... 25

Table 6: $\quad$ Salary Trends in ARL Nonuniversity Libraries ........................................................................26

\section{ARL UnIVERSITY LIBRARIES}

Table 7: $\quad$ Filled Positions; Average, Median, and Beginning Salaries; and Average Years of Experience in ARL University Libraries, FY 2010-2011..

Table 8: Beginning Professional Salaries in ARL University Libraries Rank Order Table, FY 2009-2010

Table 9: Beginning Professional Salaries in ARL University Libraries Rank Order Table, FY 2010-2011 
Table 10: Median Professional Salaries in ARL University Libraries

Rank Order Table, FY 2009-2010

Table 11: Median Professional Salaries in ARL University Libraries

Rank Order Table, FY 2010-2011 35

Table 12: $\quad$ Average Professional Salaries in ARL University Libraries

Rank Order Table, FY 2009-2010 36

Table 13: $\quad$ Average Professional Salaries in ARL University Libraries

Rank Order Table, FY 2010-2011 .37

Table 14: Average, Median, and Beginning Professional Salaries in ARL University Libraries

Summary of Rankings, FYs 2007-2008 to 2010-2011 38

Table 15: Distribution of Professional Staff in ARL University Libraries

by Salary and Position, FY 2010-2011

Table 16: Distribution of Professional Staff in ARL University Libraries

by Salary, Sex, and Position, FY 2010-2011

Table 17: Number and Average Salaries of ARL University Librarians

by Position and Sex, FY 2010-2011.

Table 18: Number and Average Years of Experience of ARL University Librarians

by Position and Sex, FY 2010-2011

Table 19: $\quad$ Number and Average Salaries of ARL University Librarians

by Years of Experience and Sex, FY 2010-2011

Table 20: $\quad$ Average Salaries of ARL University Librarians

by Position and Years of Experience, FY 2010-2011

Table 21: Number and Average Salaries of ARL University Librarians

by Position and Type of Institution, FY 2010-2011

Table 22: Years of Experience of ARL University Librarians

by Position and Type of Institution, FY 2010-2011

Table 23: Number and Average Salaries of ARL University Librarians

by Position and Size of Professional Staff, FY 2010-2011 50

Table 24: Years of Experience of ARL University Librarians

by Position and Size of Professional Staff, FY 2010-2011 .51

Table 25: Average Salaries of ARL University Librarians

by Position and Geographic Region, FY 2010-2011 .52 
ARL University Libraries by Geographic Region

\section{US ARL UNIVERSITY LIBRARIES}

Table 26: $\quad$ Average Salaries of US ARL University Librarians by Position and Years of Experience, FY 2010-2011

Table 27: Number and Average Salaries of Minority US ARL University Librarians by Position and Sex, FY 2010-2011

Table 28: Number and Average Years of Experience of Minority US ARL University Librarians by Position and Sex, FY 2010-2011 58

Table 29: Number and Average Salaries of US ARL University Librarians by Years of Experience and Sex, FY 2010-2011 .59

Table 30: Number and Average Salaries of Minority US ARL University Librarians by Years of Experience and Sex, FY 2010-2011

\section{Canadian ARL University LibraRies}

Table 31: Filled Positions; Average, Median, and Beginning Professional Salaries; and Average Years of Professional Experience in Canadian ARL University Libraries, FY 2010-2011 .........62

Table 32: Number and Average Salaries of Canadian ARL University Librarians by Position and Sex, FY 2010-2011

Table 33: Number and Average Years of Experience of Canadian ARL University Librarians by Position and Sex, FY 2010-2011 64

Table 34: Number and Average Salaries of Canadian ARL University Librarians by Years of Experience and Sex, FY 2010-2011

\section{ARL University Medical Libraries}

Table 35: Filled Positions; Average, Median, and Beginning Salaries; and Average Years of Experience in ARL University Medical Libraries, FY 2010-2011

Table 36: Beginning Professional Salaries in ARL University Medical Libraries

Rank Order Table, FY 2010-2011

Table 37: Median Professional Salaries in ARL University Medical Libraries

Rank Order Table, FY 2010-2011

Table 38: $\quad$ Average Professional Salaries in ARL University Medical Libraries

Rank Order Table, FY 2010-2011 
Table 39: Number and Average Salaries of ARL University Medical Librarians

by Position and Sex, FY 2010-2011

Table 40: Number and Average Years of Experience of ARL University Medical Librarians

by Position And Sex, FY 2010-2011 .74

Table 41: Number and Average Salaries of ARL University Medical Librarians by Years of Experience and Sex, FY 2010-2011 75

\section{ARL University LaW Libraries}

Table 42: Filled Positions; Average, Median, and Beginning Salaries; and Average Years of Experience in ARL University Law Libraries, FY 2010-2011.

Table 43: Beginning Professional Salaries in ARL University Law Libraries

Rank Order Table, FY 2010-2011

Table 44: Median Professional Salaries in ARL University Law Libraries

Rank Order Table, FY 2010-2011

Table 45: $\quad$ Average Professional Salaries in ARL University Law Libraries

Rank Order Table, FY 2010-2011

Table 46: $\quad$ Number and Average Salaries of ARL University Law Librarians

by Position and Sex, FY 2010-2011

Table 47: Number and Average Years of Experience of ARL University Law Librarians

by Position And Sex, FY 2010-2011 84

Table 48: Number and Average Salaries of ARL University Law Librarians

by Years of Experience and Sex, FY 2010-2011

UNIVERSITY LIBRARY QUESTIONNAIRE AND INSTRUCTIONS 87

NONUNIVERSITY LibraRy QUESTIONNAIRE AND INSTRUCTIONS . 101

Footnotes to the ARL Annual Salary Survey, 2010-2011 107

ARL Member Libraries as of January 1, 2011 


\section{Salary Survey Trends 2010-2011}

The ARL Annual Salary Survey 2010-2011 reports salary data for all professional staff working in Association of Research Libraries (ARL) member libraries. ARL represents the interests of libraries that serve major North American research institutions. The Association operates as a forum for the exchange of ideas and as an agent for collective action to influence forces affecting the ability of these libraries to meet the future needs of scholarship. The ARL Statistics and Assessment program, which produces the Salary Survey, is organized around collecting, analyzing, and distributing quantifiable information describing the characteristics of research libraries. The ARL Annual Salary Survey is the most comprehensive and thorough guide to current salaries in large US and Canadian academic and research libraries and is a valuable management and research tool.

Data for 10,037 professional staff members were reported this year for the 115 ARL university libraries, including their law and medical libraries ( 974 staff members reported by 73 medical libraries and 734 staff members reported by 77 law libraries). For the 10 nonuniversity ARL members, data were reported for 3,709 professional staff members.

The tables are organized in seven major sections. The first section includes Tables 1 through 4 , which report salary figures for all professionals working in ARL member libraries, including law and medical library data. The second section includes salary information for the 10 nonuniversity research libraries of ARL. The third section, entitled "ARL University Libraries," reports data in Tables 7 through 25 for the "general" library system of the university ARL members, combining US and Canadian data but excluding law and medical data. The fourth section, composed of Tables 26 through 30, reports data on US ARL university library members excluding law and medical data. The fifth section (Tables 31-34) reports data on Canadian ARL university libraries excluding law and medical data. The sixth section (Tables 35-41) and the seventh section (Tables 42-48) report on medical and law libraries, respectively, combining US and Canadian data.

The university population is generally treated in three distinct groups: staff in the "general" library system, staff in the university medical libraries, and staff in the university law libraries. Any branch libraries for which data were received, other than law and medical, are included in the "general" category, whether or not those libraries are administratively independent. Footnotes for many institutions provide information on branch inclusion or exclusion.

In all tables where data from US and Canadian institutions are combined, Canadian salaries are converted into US dollar equivalents at the rate of 1.0556 Canadian dollars per US dollar. ${ }^{1}$ Tables 4 and 31 through 34, however, pertain exclusively to staff in Canadian university libraries, so salary data in those tables are expressed in Canadian dollars.

\footnotetext{
1 This is the average monthly noon exchange rate published in the Bank of Canada Review for the period July 2009-June 2010 and is used in converting figures that are shown effective as of 1 July 2010. This information can be accessed at: http:/ / www.bankofcanada.ca/en/rates / exchange.html.
} 


\section{RACE AND ETHNICITY}

There were 1,266 minority professional staff reported in 99 US ARL university libraries, including law and medical libraries. ${ }^{2}$ Note that the data for minority professionals comes only from the US ARL university libraries following the Equal Employment Opportunity Commission (EEOC) definitions; Canadian law prohibits the identification of Canadians by ethnic category.

Currently, $14.2 \%$ of the professional staff in US ARL university libraries (including law and medical libraries) belong to one of the four non-Caucasian categories for which ARL keeps records. The percentage of minorities in managerial or leadership positions in the largest US academic libraries is far lower: $6.4 \%$ are directors (7 out of 110), $6.5 \%$ are associate directors (21 out of 325), 7.7\% are assistant directors (13 out of 168), and 9.1\% (41 out of 452) are the head of a branch library (see Table 27). Figure 1, below, depicts the overall racial/ethnic distribution of professional staff in US ARL university libraries: Caucasian/Other 85.8\%, Asian/Pacific Islander 6.6\%, Black 4.4\%, Hispanic 2.8\%, and American Indian/Alaskan Native $0.4 \%$.

\section{Figure 1: Ethnicity/Race of Professional Staff in US ARL University Libraries, FY 2010-2011}

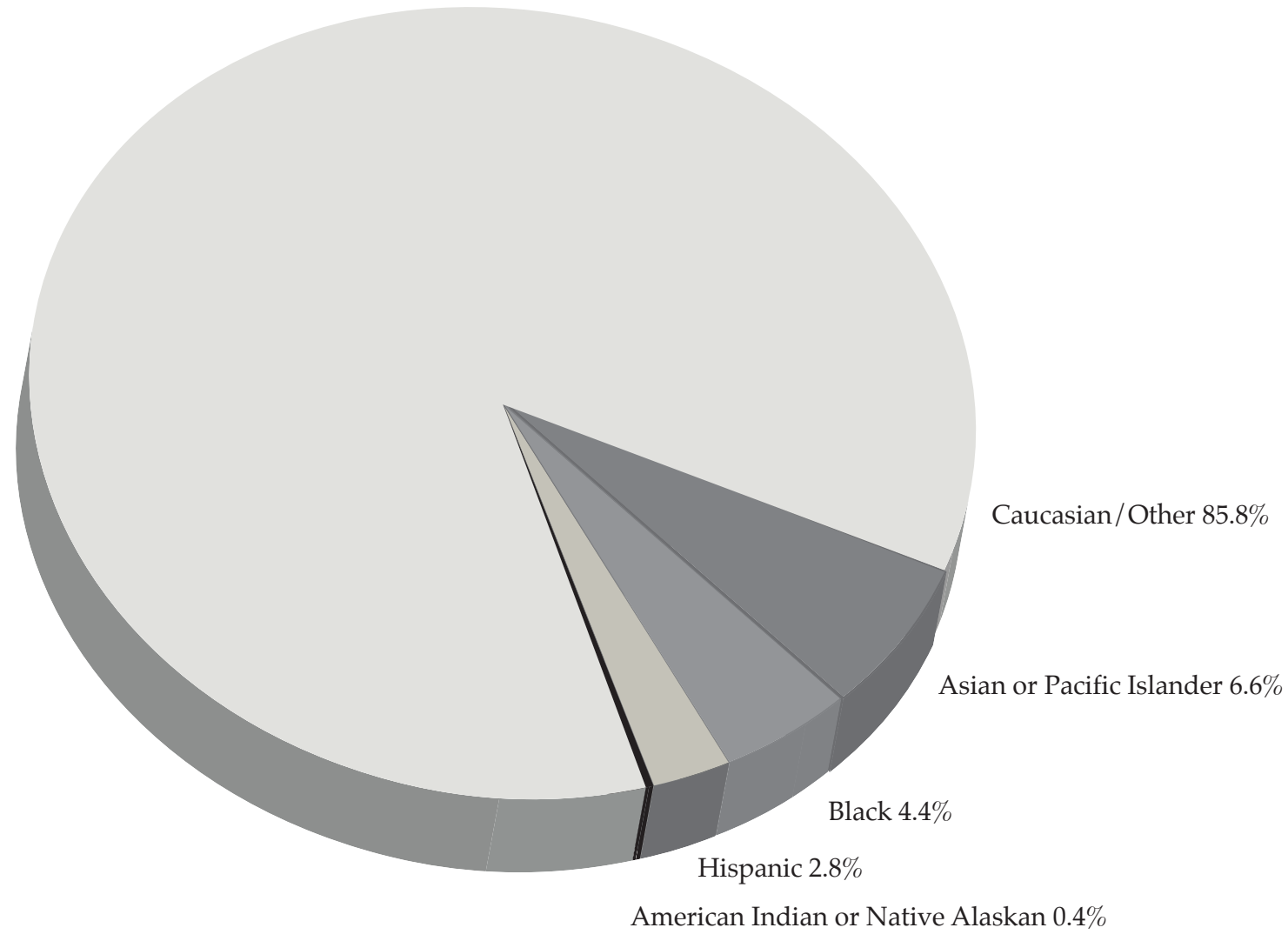

2 Some US institutions offer their librarians the option of not reporting race and ethnicity; others forbid the tracking of racial and ethnic classification altogether. See Footnotes. 
Minority professional staff in US ARL university libraries continues to be disproportionately distributed across the country. Using Figure 2, we can compare the number of minority staff with other staff, region by region. These patterns of distribution have been relatively stable for the entire history of ARL's data-collection experience. Minorities are underrepresented by over 34\% in the East South Central region and by more than 28\% in the West North Central region (see Table 25 for a definition of the regions). Proportionately to other regions, there are more minorities in the Pacific, South Atlantic, West South Central, and Middle Atlantic regions.

Figure 2: Minority Professionals by Region in US ARL University Libraries, FY 2010-2011

\begin{tabular}{|c|c|c|c|c|c|c|c|c|c|c|c|}
\hline & $\begin{array}{l}\text { New } \\
\text { England }\end{array}$ & $\begin{array}{l}\text { Middle } \\
\text { Atlantic }\end{array}$ & $\begin{array}{l}\text { East } \\
\text { North } \\
\text { Central }\end{array}$ & $\begin{array}{l}\text { West } \\
\text { North } \\
\text { Central }\end{array}$ & $\begin{array}{l}\text { South } \\
\text { Atlantic }\end{array}$ & $\begin{array}{c}\text { East } \\
\text { South } \\
\text { Central }\end{array}$ & $\begin{array}{l}\text { West } \\
\text { South } \\
\text { Central }\end{array}$ & Mountain & Pacific & TOTAL & $\%$ \\
\hline \multirow{2}{*}{\multicolumn{12}{|c|}{$\begin{array}{l}\text { Race/Ethnicity } \\
\text { Category }\end{array}$}} \\
\hline & & & & & & & & & & & \\
\hline Black & 31 & 72 & 74 & 24 & 109 & 22 & 28 & 7 & 28 & 395 & $31 \%$ \\
\hline Hispanic & 21 & 44 & 27 & 10 & 39 & 6 & 40 & 20 & 43 & 250 & $20 \%$ \\
\hline Asian & 79 & 105 & 69 & 25 & 84 & 9 & 40 & 21 & 155 & 587 & $46 \%$ \\
\hline $\mathbf{A I} / \mathbf{A N}^{*}$ & 3 & 3 & 15 & 4 & 1 & 0 & 1 & 7 & 0 & 34 & $3 \%$ \\
\hline Minority Total & 134 & 224 & 185 & 63 & 233 & 37 & 109 & 55 & 226 & 1,266 & $100 \%$ \\
\hline \multicolumn{12}{|l|}{ Minority } \\
\hline Percent & $10.60 \%$ & $17.70 \%$ & $14.60 \%$ & $5.00 \%$ & $18.40 \%$ & $2.90 \%$ & $8.60 \%$ & $4.30 \%$ & $17.90 \%$ & & \\
\hline \multicolumn{12}{|l|}{ Nonminority } \\
\hline Total & 1,100 & 1,267 & 1,390 & 531 & 1,216 & 342 & 591 & 430 & 778 & 7,645 & $100 \%$ \\
\hline \multicolumn{12}{|l|}{ Nonminority } \\
\hline Percent & $14.40 \%$ & $16.60 \%$ & $18.20 \%$ & $6.90 \%$ & $15.90 \%$ & $4.50 \%$ & $7.70 \%$ & $5.60 \%$ & $10.20 \%$ & & \\
\hline \multicolumn{12}{|l|}{ Regional } \\
\hline \multicolumn{12}{|l|}{ Percent } \\
\hline Total staff & $13.80 \%$ & $16.70 \%$ & $17.70 \%$ & $6.70 \%$ & $16.30 \%$ & $4.30 \%$ & $7.90 \%$ & $5.40 \%$ & $11.30 \%$ & & \\
\hline \multicolumn{12}{|l|}{ Proportional } \\
\hline Minority & & & & & & & & & & & \\
\hline Representation & $-26.44 \%$ & $6.76 \%$ & $-19.63 \%$ & $-28.35 \%$ & $15.71 \%$ & $-34.67 \%$ & $11.37 \%$ & $-22.76 \%$ & $75.42 \%$ & & \\
\hline
\end{tabular}

* American Indian/Alaskan Native

According to Figure 3 below, $68.6 \%$ of female professional staff in US ARL university libraries are members of the four racial/ethnic groups in Figure 2, whereas $62.4 \%$ of female professional staff are members of the Caucasian/Other racial/ethnic group. The overall gender balance in the 115 Canadian and US university libraries (including law and medical libraries) is 35.9\% male and 64.1\% female. See Figure 2, above, and Figure 3, below, for more detail on race/ethnic and gender distribution. 
Figure 3: Race/Ethnicity and Sex Distribution of Professional Staff in ARL University Libraries, FY 2010-2011

\begin{tabular}{|c|c|c|c|c|c|}
\hline \multicolumn{6}{|c|}{ UNITED STATES } \\
\hline & \multicolumn{2}{|c|}{ Men } & \multicolumn{2}{|c|}{ Women } & \multirow[t]{2}{*}{ Total } \\
\hline & Number of Staff & Percent of Total & Number of Staff & Percent of Total & \\
\hline Main & 2,785 & $37.8 \%$ & 4,587 & $62.2 \%$ & 7,372 \\
\hline Medical & 257 & $29.7 \%$ & 608 & $70.3 \%$ & 865 \\
\hline Law & 231 & $33.6 \%$ & 457 & $66.4 \%$ & 688 \\
\hline Minority* & 397 & $31.4 \%$ & 869 & $68.6 \%$ & 1,266 \\
\hline Non-minority & 2,871 & $37.6 \%$ & 4,774 & $62.4 \%$ & 7,645 \\
\hline All & 3,268 & $36.7 \%$ & 5,643 & $63.3 \%$ & 8,911 \\
\hline \multicolumn{6}{|c|}{ CANADA } \\
\hline & \multicolumn{2}{|c|}{ Men } & \multicolumn{2}{|c|}{ Women } & Total \\
\hline & Number of Staff & Percent of Total & Number of Staff & Percent of Total & \\
\hline Main & 306 & $32.0 \%$ & 651 & $68.0 \%$ & 957 \\
\hline Medical & 10 & $9.2 \%$ & 99 & $90.8 \%$ & 109 \\
\hline Law & 15 & $32.6 \%$ & 31 & $67.4 \%$ & 46 \\
\hline All & 331 & $29.8 \%$ & 781 & $70.2 \%$ & 1,112 \\
\hline \multicolumn{6}{|c|}{ United States and Canada (Combined) } \\
\hline & \multicolumn{2}{|c|}{ Men } & \multicolumn{2}{|c|}{ Women } & Total \\
\hline & Number of Staff & Percent of Total & Number of Staff & Percent of Total & \\
\hline Main & 3,091 & $37.1 \%$ & 5,238 & $62.9 \%$ & 8,329 \\
\hline Medical & 267 & $27.4 \%$ & 707 & $72.6 \%$ & 974 \\
\hline Law & 246 & $33.5 \%$ & 488 & $66.5 \%$ & 734 \\
\hline All & 3,604 & $35.9 \%$ & 6,433 & $64.1 \%$ & 10,037 \\
\hline
\end{tabular}

* Includes staff in medical and law libraries.

Note: There are two US institutions that did not report race/ethnicity data; therefore, the totals will not aggregate to the total needed for the US and Canadian sub-totals to equal the figure displayed in the combined total.

ARL recognizes the difficulties that the profession has in attracting a diverse workforce and continues to work actively in the development of workplace climates that embrace diversity. The ARL Diversity Programs, through its Leadership and Career Development Program and the Initiative to Recruit a Diverse Workforce, emphasize ARL's and its members' commitment to creating a diverse academic and research library community to better meet the new challenges of global competition and changing demographics. Further, the Diversity Programs focus on issues surrounding work relationships in libraries while considering the impact of diversity on library services, interactions with library users, and the development of collections. More information about the Diversity Programs can be found at http://www.arl.org/diversity/. 
ClimateQUAL ${ }^{\circledR}$ is an assessment initiative that focuses on some of the same issues. It is the Statistics and Measurement program's tool that assesses organizational climate and diversity in libraries. ClimateQUAL $\AA$ helps libraries plumb the dimensions of climate and organizational culture important for a healthy organization in a library setting. The ClimateQUAL ${ }^{\circledR}$ survey addresses climate issues such as diversity, teamwork, learning, and fairness, as well as current managerial practices, and staff attitudes and beliefs. Libraries use their ClimateQUAL $®$ data to improve their organizational climate and diversity culture for delivering superior services to the communities they serve. More information about ClimateQUAL® can be found at http://www. climatequal.org.

\section{GENDER DATA}

Many readers of previous surveys have inquired about evidence of gender-based salary differentials in ARL libraries. Additionally, data on salary comparisons for directors also are frequently requested. Since 2008-2009, the average salary for female directors was slightly higher than that of their male counterparts. However, this year the trend was reversed, with male directors earning more than female directors (see Table 17); furthermore, the number of women in the top administrative library position decreased to 66 out of 110 total director positions reported in 2010-2011 (see Table 17).

In keeping with previous years, the 2010-2011 data show that salaries for women in US ARL university libraries have not yet met parity with that of men (see Table 17). In 2010-2011 the overall salary for women was only $96.05 \%$ of that of men for the 110 ARL university libraries (compared to 96.3\% in 2009-2010). This suggests a slight regression in the slow, long-term trend towards closure of the gender gap in ARL libraries — in 1980-1981, women in ARL libraries made roughly $87 \%$ that of men.

Table 17 displays 27 job categories; females earn more than their male counterparts in just 12 of the 27 categories listed. Table 18 provides average years of professional experience for many of the same staffing categories for which salary data are shown in Table 17, revealing that experience differentials may explain some differences within specific job categories. Women have more experience in all but two of the twelve job categories in which they average higher pay. However, there are four other categories in which women, on average, have more experience and less pay: Director, Assistant Director, Department Head-Documents and Maps, and Department Head-Other. Table 19 further reveals that the average salary for men is consistently higher than the average salary for women in all ten experience cohorts. Among minority librarians, the average salary for minority men is higher than that for minority women in eight of the ten experience cohorts (see Table 30).

There is a sense that the gender gap persists in academe in areas beyond the library and that a renewed commitment to resolve the problem is needed. ${ }^{3}$ A variety of reasons have been offered as to why these trends persist, most notably the perception that work is peripheral in a woman's life and, consequently, femaledominated professions are undervalued. Librarianship is predominantly and persistently a woman's profession.

3 There are many instances citing the continuation of gender inequity in academia. See, for example: Mary Ann Mason, "Still Earning Less," Chronicle of Higher Education 13 January 2010 http:/ / chronicle.com/article/Still-Earning-Less / 63482/; Katherine Mangan, "Women in Academic Medicine: Equal to Men, Except in Pay," Chronicle of Higher Education 31 March 2010 http: / chronicle.com/article/ Womenin-Academic-Medicine-/64892/; Paula Wasley, “Gender Gap in Pay Widens Over Time,” Chronicle of Higher Education 4 May 2007 http:/ / chronicle.com/article/Gender-Gap-in-Pay-Widens-Over/9208/; Denise K. Manger's articles in the Chronicle of Higher Education, "Faculty Salaries Increased 3.7\% in 1999-2000" (14 April 2000: A20) and “Faculty Salaries are Up 3.6\%, Double the Rate of Inflation" (23 April 1999: A16); D. W. Miller, "Salary Gap Between Male and Female Professors Grows Over the Years, Study Suggests," Chronicle of Higher Education, Today's News, 27 April 2000; and Yolanda Moses, "Salaries in Academe: The Gender Gap Persists," Chronicle of Higher Education 12 December 1997: A60. 
The scarcity of men in the profession has been well documented in many studies-the largest percentage of men employed in ARL libraries was 38.2\% in 1980-1981; since then men have consistently represented about 35\% of the professional staff in ARL libraries.

\section{The Functional Specialist Breakdown}

In 2004, the ARL Statistics and Measurement Committee accepted a proposal from the ACRL Personnel Administrators and Staff Development Officers Discussion Group to break down the Functional Specialist category (FSPEC). The group's major concern was that so many different types of positions, with their varying job descriptions and salaries, were being labeled with the code FSPEC that data reported for the category were beginning to lose meaning. For each position that would have been labeled FSPEC in past years, the proposal offered ARL institutions two options: either use one of eight new codes to describe that position; or, if none of the eight new codes could adequately describe that position, use FSPEC. As seen in Figure 4, 17.3\% of Functional Specialists in all libraries did not use an alternative code, an increase over the 2009-2010 figures. As in 2009-2010, Archivists and Information Technology specialists comprised the largest percentage of Functional Specialists who used an alternative code $(61.5 \%)$.

Figure 4: Distribution of Functional Specialist Job Sub-Codes by Type of Library

\begin{tabular}{|c|c|c|c|c|c|c|c|c|}
\hline \multirow{2}{*}{ Position } & \multicolumn{2}{|c|}{ Main } & \multicolumn{2}{|c|}{ Medical } & \multicolumn{2}{|c|}{ Law } & \multicolumn{2}{|c|}{ All } \\
\hline & No. & Percent & No. & Percent & No. & Percent & No. & Percent \\
\hline Archivists & 476 & $22.3 \%$ & 20 & $10.9 \%$ & 6 & $11.1 \%$ & 502 & $21.1 \%$ \\
\hline Business Manager & 148 & $6.9 \%$ & 17 & $9.2 \%$ & 2 & $3.7 \%$ & 167 & $7.0 \%$ \\
\hline Human Resources & 83 & $3.9 \%$ & 1 & $0.5 \%$ & 0 & $0.0 \%$ & 84 & $3.5 \%$ \\
\hline IT, Systems & 397 & $18.6 \%$ & 44 & $23.9 \%$ & 13 & $24.1 \%$ & 454 & $19.1 \%$ \\
\hline IT, Web Developer & 153 & $7.2 \%$ & 18 & $9.8 \%$ & 9 & $16.7 \%$ & 180 & $7.6 \%$ \\
\hline IT, Programmer & 294 & $13.8 \%$ & 32 & $17.4 \%$ & 0 & $0.0 \%$ & 326 & $13.7 \%$ \\
\hline Media Specialist & 108 & $5.1 \%$ & 7 & $3.8 \%$ & 4 & $7.4 \%$ & 116 & $4.9 \%$ \\
\hline Preservation & 129 & $6.0 \%$ & 3 & $1.6 \%$ & 1 & $1.9 \%$ & 133 & $5.6 \%$ \\
\hline Other Functional Specialists & 350 & $16.4 \%$ & 42 & $22.8 \%$ & 19 & $35.2 \%$ & 411 & $17.3 \%$ \\
\hline Total & 2,138 & & 184 & & 54 & & 2,376 & \\
\hline
\end{tabular}

Figure 5, below, displays the average salaries of the subcategories by position and sex (law and medical libraries not included) in the same fashion as Table 17. The salaries in each of the sub-categories deviate widely from the combined Functional Specialist average salary of $\$ 65,562$. Human resource specialists have the highest average of all subcategories, with an average salary of $\$ 74,293$; media/multimedia specialists have the lowest average salary of $\$ 56,656$. 
Figure 5: Distribution of Functional Specialist Job Sub-Codes' Average Salaries by Sex

\begin{tabular}{|l|c|r|r|r|r|r|}
\hline \multirow{2}{*}{ Position } & \multicolumn{2}{|c|}{ Women } & \multicolumn{2}{c|}{ Men } & \multicolumn{2}{c|}{ Total } \\
\cline { 2 - 6 } & \multirow{2}{*}{ Salary } & No. & Salary & No. & Salary & No. \\
\cline { 1 - 6 } Archivists & \multirow{2}{*}{58,579} & 304 & 62,798 & 172 & 60,103 & 476 \\
Business Manager & 71,545 & 94 & 69,483 & 54 & 70,793 & 148 \\
Human Resources & 73,821 & 70 & 76,836 & 13 & 74,293 & 83 \\
IT, Systems & 67,504 & 134 & 67,104 & 263 & 67,239 & 397 \\
IT, Web Developer & 63,210 & 65 & 65,032 & 88 & 64,258 & 153 \\
IT, Programmer & 66,506 & 91 & 69,418 & 203 & 68,516 & 294 \\
Media Specialist & 56,837 & 51 & 56,495 & 57 & 56,656 & 108 \\
Preservation & 63,646 & 89 & 66,466 & 40 & 64,521 & 129 \\
\hline Other Functional Specialists & 63,264 & 233 & 64,515 & 117 & 63,682 & 350 \\
\hline All Functional Specialists & $\mathbf{6 4 , 9 9 0}$ & $\mathbf{1 , 1 3 1}$ & $\mathbf{6 6 , 4 6 1}$ & $\mathbf{1 , 0 0 7}$ & $\mathbf{6 5 , 5 6 2}$ & $\mathbf{2 , 1 3 8}$ \\
\hline
\end{tabular}

In regards to the gender gap in ARL libraries discussed in the previous section, it is worth noting that the average salaries of men are higher than those of women in six out of the nine categories in Figure 5.

\section{Institutional Characteristics AND SAlaries}

\section{A. Public and Private Institutions}

The gap between salaries paid in private US ARL university libraries and those paid in publicly supported US university libraries increased in 2010-2011 to 7.1\%, with librarians at private institutions earning an average of $\$ 4,921$ more than their peers at public institutions. Out of 18 job categories, only in two (Head of Serials and Head of Computer Systems) did librarians in public institutions earn more than their peers employed in private institutions (see Table 21).

\section{B. Library Size}

Library size, as measured by the number of professional staff, is another significant determinant of salary. As a rule, the largest libraries tend to pay the highest average salaries, not only overall, but for specific positions, as well. However, in 2010-2011, the libraries with between 75 and 110 staff reported the highest average salary, $\$ 74,158$, followed by the largest libraries, i.e., those with more than 110 staff, which reported the next highest average salary, $\$ 73,863$ (see Table 23). The gap between the highest paying cohort and the lowest paying cohort decreased in 2010-2011 to $\$ 3,994$. The cutoff staffing levels used to determine the largest cohort of libraries, after declining in every year since 1995-1996, continued to hold steady at 110 in 2010-2011. ${ }^{4}$

\section{Geographic Area}

In 2010-2011, the highest average salaries were found in Canada $(\$ 83,424)$ followed by New England $(\$ 77,452)$ with salaries in the Pacific region $(\$ 75,823)$ coming in third (see Table 25$)$. The Canadian average salary has

4 In 1995-1996, the largest cohort of libraries was determined based on staff over 124; in 1996-1998, over 120; in 1998-1999, over 115; and since 1999-2000, over 110. See Table 23. 
not been this high since $2008-2009$ when it was $\$ 82,295$. This sharp increase in Canadian salaries is due to fluctuations in the currency exchange rate. For the 2010-2011 survey period the Canadian currency exchange rate is 1.0556. The East South Central region had the lowest average salary: $\$ 62,842$.

\section{Rank Structure}

Rank structure provides a useful framework for examining professional salaries in ARL university libraries. Figure 6, below, displays average salary and years of experience in the most commonly used rank structures. Readers should be aware that not all individuals have a rank that fits into the rank structure the library utilizes. Most commonly, directors may have no rank (or a rank outside the structure) and it is common for non-librarians included in the survey (business officers, personnel staff, computer specialists, liaisons, etc.) to be unranked, as well.

The pattern of relationships between rank and salary seen in past years continues: with higher rank associated with higher average years of experience and a correspondingly higher salary. 5,722 of the 8,329 librarians in ARL university member libraries occupy a rank within these three most commonly found ranking systems, and the largest number of professionals $(3,183)$ occupy a position in a four-step rank structure.

Figure 6: Average Salaries and Average Years of Experience of Library Professionals in Libraries with Three, Four, and Five Step Rank Structures, FY 2010-2011

\begin{tabular}{|l|c|c|c|c|c|c|}
\hline & \multicolumn{2}{|c|}{ Three-Step } & \multicolumn{2}{c|}{ Four-Step } & \multicolumn{2}{c|}{ Five-Step } \\
\hline & Salary & Experience & Salary & Experience & Salary & Experience \\
Librarian 1 & 59,888 & 9.2 & 53,813 & 7.9 & 53,176 & 7.7 \\
Librarian 2 & 69,935 & 17.6 & 60,214 & 13.1 & 59,922 & 13.0 \\
Librarian 3 & 87,641 & 25.4 & 72,896 & 20.4 & 68,968 & 17.5 \\
Librarian 4 & & 88,116 & 26.3 & 87,744 & 23.6 \\
Librarian 5 & \multicolumn{2}{|c|}{1,475} & \multicolumn{2}{|c|}{3,183} & 103,069 & 29.1 \\
\hline No. of Staff & \multicolumn{3}{|c|}{} & \multicolumn{3}{|c|}{$\mathbf{1 , 0 6 4}$} \\
\hline
\end{tabular}

\section{INFLATION EFFECT}

Tables 2 and 6 reveal changes in beginning professional and median salaries as reported by both university and nonuniversity research libraries as well as the US Bureau of Labor's Cost of Living Index (CPI-All Urban Consumers). Table 3 is similar to Table 2, but reports data only on US libraries. Table 4 shows trend data for Canadian libraries and compares them to the changes in the Canadian Consumer Price Index (Consumer Price Index for Canada, all-items, not seasonally adjusted). Tables 2, 3, and 4 include law and medical library staff in ARL university libraries. In contrast to 2009-2010, these tables indicate that the purchasing power of professionals (in both the United States and Canada) employed in ARL libraries kept pace with inflation.

The median salary for US ARL university libraries in 2010 increased to $\$ 65,000$ (see Table 3). This modest salary increase barely kept pace with the rebounding economy, which saw the US CPI increase by $1.2 \%$ (see Table 3). ${ }^{5}$ Likewise, Canadian salaries (reported in Canadian dollars) also barely surpassed inflation: the Canadian CPI

5 CPI data retrieved from the US Department of Labor, Bureau of Labor Statistics' Consumer Price Index-All Urban Consumers (US All items, 1982-84=100 - CUUR0000SA0) available online at http:/ / www.bls.gov/ data/. 
increased $1.8 \%$, while median salaries in Canadian university libraries increased to $\$ 82,251$ (Canadian dollars, see Table 4). ${ }^{6}$ The sharp difference in the exchange rates between 2009-2010 (1.1667 Canadian per US dollar) and 2010-2011 (1.0556 Canadian per US dollar) contributed to these changes.

The median beginning salary (BPS) for university ARL librarians rebounded back to 2008-2009 levels to $\$ 44,004$ in 2010-2011 (see Table 2). Table 6 shows that nonuniversity librarians also experienced increases in their median and beginning salaries in $2010-2011$, which increased to $\$ 95,020$ and $\$ 51,135$, respectively.

Readers are reminded that these data reflect only salaries, and that there are other compensation issues which may have influenced the pattern of salaries in various institutions. In addition, a highly standardized structure for capturing data has been used, which may portray results in a way that cannot be fully representative of a local situation.

Martha Kyrillidou

Shaneka Morris

Association of Research Libraries

September 30, 2011

6 The source for Canadian CPI data is Table 5: The Consumer Price Index for Canada (All-Items, Not Seasonally Adjusted, Historical Data) published in The Daily, a Statistics Canada publication, available online at http:/ / www.statcan.gc.ca/pub/62-001-x/2009010/t040-eng.htm. 
16 · ARL Annual Salary Survey 2010-2011 
Salary LeVels for Staff in ARL Libraries

TABLes 1-4 



\section{Table 1: Distribution by Salary Level}

Figures in columns headed by fiscal year show the number of filled professional positions. Columns headed by Cum. \% show the percentage of all filled positions with salaries equal to or more than the beginning of each salary range.

Note: Canadian salaries are expressed in US dollars. Data includes medical and law libraries.

\begin{tabular}{|c|c|c|c|c|c|c|c|c|}
\hline \multirow[b]{2}{*}{ Salary Range } & \multicolumn{4}{|c|}{ University Libraries } & \multicolumn{4}{|c|}{ Nonuniversity Libraries } \\
\hline & $\begin{array}{c}\text { FY } \\
2009-2010 \\
\end{array}$ & $\begin{array}{r}\text { Cum. } \\
\% \\
\end{array}$ & $\begin{array}{c}\text { FY } \\
\text { 2010-2011 } \\
\end{array}$ & $\begin{array}{r}\text { Cum. } \\
\% \\
\end{array}$ & $\begin{array}{c}\text { FY } \\
2009-2010 \\
\end{array}$ & $\begin{array}{r}\text { Cum. } \\
\% \\
\end{array}$ & $\begin{array}{c}\text { FY } \\
\text { 2010-2011 } \\
\end{array}$ & $\begin{array}{r}\text { Cum. } \\
\% \\
\end{array}$ \\
\hline 300,000 and above & 6 & $0.1 \%$ & 6 & $0.1 \%$ & 0 & $0.0 \%$ & 0 & $0.0 \%$ \\
\hline 250,000-299,999 & 12 & $0.2 \%$ & 11 & $0.2 \%$ & 1 & $0.0 \%$ & 0 & $0.0 \%$ \\
\hline $200,000-250,000$ & 37 & $0.5 \%$ & 42 & $0.6 \%$ & 3 & $0.1 \%$ & 3 & $0.1 \%$ \\
\hline $175,000-199,999$ & 58 & $1.1 \%$ & 56 & $1.1 \%$ & 6 & $0.3 \%$ & 7 & $0.3 \%$ \\
\hline $150,000-174,999$ & 80 & $1.9 \%$ & 87 & $2.0 \%$ & 284 & $7.7 \%$ & 315 & $8.8 \%$ \\
\hline $140,000-149,999$ & 52 & $2.4 \%$ & 70 & $2.7 \%$ & 105 & $10.5 \%$ & 116 & $11.9 \%$ \\
\hline $130,000-139,999$ & 80 & $3.2 \%$ & 99 & $3.7 \%$ & 237 & $16.7 \%$ & 218 & $17.8 \%$ \\
\hline $120,000-129,999$ & 127 & $4.4 \%$ & 141 & $5.1 \%$ & 170 & $21.1 \%$ & 252 & $24.6 \%$ \\
\hline $110,000-119,999$ & 193 & $6.3 \%$ & 253 & $7.6 \%$ & 370 & $30.9 \%$ & 331 & $33.5 \%$ \\
\hline $100,000-109,999$ & 328 & $9.5 \%$ & 402 & $11.6 \%$ & 383 & $40.9 \%$ & 422 & $44.9 \%$ \\
\hline 95,000-99,999 & 287 & $12.3 \%$ & 304 & $14.7 \%$ & 301 & $48.8 \%$ & 205 & $50.4 \%$ \\
\hline $90,000-94,999$ & 337 & $15.6 \%$ & 312 & $17.8 \%$ & 144 & $52.6 \%$ & 210 & $56.1 \%$ \\
\hline $85,000-89,999$ & 418 & $19.7 \%$ & 452 & $22.3 \%$ & 276 & $59.8 \%$ & 206 & $61.6 \%$ \\
\hline $80,000-84,999$ & 546 & $25.1 \%$ & 575 & $28.0 \%$ & 201 & $65.1 \%$ & 241 & $68.1 \%$ \\
\hline $79,000-79,999$ & 121 & $26.3 \%$ & 122 & $29.2 \%$ & 48 & $66.4 \%$ & 63 & $69.8 \%$ \\
\hline $78,000-78,999$ & 143 & $27.7 \%$ & 118 & $30.4 \%$ & 48 & $67.6 \%$ & 5 & $69.9 \%$ \\
\hline $76,000-77,999$ & 261 & $30.2 \%$ & 258 & $33.0 \%$ & 49 & $68.9 \%$ & 66 & $71.7 \%$ \\
\hline $74,000-75,999$ & 321 & $33.4 \%$ & 320 & $36.1 \%$ & 89 & $71.2 \%$ & 115 & $74.8 \%$ \\
\hline $72,000-73,999$ & 285 & $36.2 \%$ & 338 & $39.5 \%$ & 123 & $74.5 \%$ & 56 & $76.3 \%$ \\
\hline $70,000-71,999$ & 323 & $39.3 \%$ & 351 & $43.0 \%$ & 29 & $75.2 \%$ & 83 & $78.6 \%$ \\
\hline $68,000-69,999$ & 400 & $43.3 \%$ & 387 & $46.9 \%$ & 97 & $77.8 \%$ & 94 & $81.1 \%$ \\
\hline $66,000-67,999$ & 370 & $46.9 \%$ & 374 & $50.6 \%$ & 89 & $80.1 \%$ & 79 & $83.2 \%$ \\
\hline $64,000-65,999$ & 451 & $51.3 \%$ & 480 & $55.4 \%$ & 89 & $82.4 \%$ & 49 & $84.6 \%$ \\
\hline $62,000-63,999$ & 400 & $55.2 \%$ & 377 & $59.1 \%$ & 114 & $85.4 \%$ & 178 & $89.4 \%$ \\
\hline $60,000-61,999$ & 530 & $60.4 \%$ & 495 & $64.1 \%$ & 93 & $87.9 \%$ & 47 & $90.6 \%$ \\
\hline $58,000-59,999$ & 430 & $64.6 \%$ & 408 & $68.1 \%$ & 46 & $89.1 \%$ & 54 & $92.1 \%$ \\
\hline $56,000-57,999$ & 487 & $69.4 \%$ & 457 & $72.7 \%$ & 120 & $92.2 \%$ & 61 & $93.7 \%$ \\
\hline $54,000-55,999$ & 459 & $73.9 \%$ & 422 & $76.9 \%$ & 44 & $93.4 \%$ & 48 & $95.0 \%$ \\
\hline $52,000-53,999$ & 496 & $78.7 \%$ & 476 & $81.6 \%$ & 84 & $95.6 \%$ & 45 & $96.2 \%$ \\
\hline $50,000-51,999$ & 439 & $83.1 \%$ & 397 & $85.6 \%$ & 87 & $97.9 \%$ & 75 & $98.2 \%$ \\
\hline $48,000-49,999$ & 446 & $87.4 \%$ & 353 & $89.1 \%$ & 13 & $98.2 \%$ & 10 & $98.5 \%$ \\
\hline $46,000-47,999$ & 368 & $91.0 \%$ & 330 & $92.4 \%$ & 16 & $98.6 \%$ & 7 & $98.7 \%$ \\
\hline $44,000-45,999$ & 326 & $94.2 \%$ & 263 & $95.0 \%$ & 11 & $98.9 \%$ & 8 & $98.9 \%$ \\
\hline $42,000-43,999$ & 217 & $96.3 \%$ & 200 & $97.0 \%$ & 14 & $99.3 \%$ & 33 & $99.8 \%$ \\
\hline $40,000-41,999$ & 175 & $98.1 \%$ & 134 & $98.3 \%$ & 17 & $99.7 \%$ & 0 & $99.8 \%$ \\
\hline $38,000-39,999$ & 85 & $98.9 \%$ & 67 & $99.0 \%$ & 0 & $99.7 \%$ & 1 & $99.8 \%$ \\
\hline $36,000-37,999$ & 52 & $99.4 \%$ & 39 & $99.4 \%$ & 2 & $99.8 \%$ & 2 & $99.9 \%$ \\
\hline $34,000-35,999$ & 38 & $99.8 \%$ & 25 & $99.6 \%$ & 3 & $99.9 \%$ & 2 & $99.9 \%$ \\
\hline less than 34,000 & 23 & $100.0 \%$ & 36 & $100.0 \%$ & 5 & $100.0 \%$ & 2 & $100.0 \%$ \\
\hline Total Positions & 10,207 & & 10,037 & & 3,748 & & 3,709 & \\
\hline Median Salary & $\$ 64,560$ & & $\$ 66,260$ & & $\$ 80,320$ & & $\$ 95,020$ & \\
\hline
\end{tabular}




\section{Table 2: Salary Trends in ARL University Libraries}

Salary figures for the current year are displayed in the context of previous years and compared to the changes in the US Consumer Price Index (CPI) to show trends in the purchasing power of median and beginning professional salaries. Salary figures and CPI numbers have been converted to adjusted indexes, using July 1984 as the base. Actual CPI data retrieved from the US Department of Labor, Bureau of Labor Statistics' Consumer Price Index-All Urban Consumers (US All items, 1982-1984=100 - CUUR0000SA0) available online at http://www. bls.gov/data/.

Note: Canadian salaries are expressed in US dollars.

\begin{tabular}{|c|c|c|c|c|c|c|c|c|}
\hline $\begin{array}{l}\text { Fiscal } \\
\text { Year }\end{array}$ & $\begin{array}{c}\text { Number of } \\
\text { Libraries }\end{array}$ & $\begin{array}{l}\text { Total } \\
\text { Staff }\end{array}$ & $\begin{array}{c}\text { Median } \\
\text { Salary }^{+}\end{array}$ & $\begin{array}{c}\text { BPS }^{\ddagger} \\
\text { Median }\end{array}$ & $\begin{array}{c}\text { Median } \\
\text { Salary Index }\end{array}$ & $\begin{array}{l}\text { BPS }^{\ddagger} \\
\text { Index }\end{array}$ & $\begin{array}{l}\text { Actual } \\
\text { CPI* }^{*}\end{array}$ & $\begin{array}{l}\text { Adjusted } \\
\text { CPI }\end{array}$ \\
\hline 2010-2011 & 115 & 10,037 & $\$ 66,260$ & $\$ 44,004$ & 253.9 & 266.7 & 218.0 & 209.8 \\
\hline 2009-2010 & 114 & 10,207 & 64,560 & 43,700 & 247.4 & 264.8 & 215.4 & 207.3 \\
\hline 2008-2009 & 113 & 10,148 & 64,823 & 44,000 & 248.4 & 266.7 & 219.9 & 211.6 \\
\hline 2007-2008 & 113 & 9,983 & 61,833 & 41,125 & 236.9 & 249.7 & 208.3 & 200.5 \\
\hline 2006-2007 & 113 & 9,824 & 59,648 & 40,000 & 228.5 & 242.4 & 203.5 & 195.9 \\
\hline 2005-2006 & 113 & 9,655 & 57,074 & 37,920 & 218.7 & 229.8 & 195.4 & 188.1 \\
\hline 2004-2005 & 113 & 9,487 & 55,250 & 36,984 & 211.7 & 224.1 & 189.4 & 182.3 \\
\hline 2003-2004 & 114 & 9,492 & 53,000 & 36,000 & 203.1 & 218.2 & 183.9 & 177.0 \\
\hline 2002-2003 & 114 & 9,469 & 51,636 & 35,000 & 197.8 & 212.1 & 180.1 & 173.3 \\
\hline 2001-2002 & 113 & 9,198 & 50,724 & 34,000 & 194.3 & 206.1 & 177.5 & 170.8 \\
\hline 2000-2001 & 112 & 8,882 & 49,068 & 32,879 & 188.0 & 199.3 & 172.8 & 166.3 \\
\hline $1999-2000$ & 111 & 8,595 & 47,377 & 31,100 & 181.5 & 188.5 & 166.7 & 160.4 \\
\hline 1998-1999 & 110 & 8,400 & 45,775 & 30,000 & 175.2 & 181.7 & 163.2 & 157.1 \\
\hline 1997-1998 & 110 & 8,414 & 44,534 & 28,500 & 170.5 & 172.6 & 160.5 & 154.5 \\
\hline 1996-1997 & 109 & 8,325 & 43,170 & 27,687 & 165.3 & 167.7 & 157.0 & 151.1 \\
\hline 1995-1996 & 108 & 8,231 & 41,901 & 27,000 & 160.5 & 163.6 & 152.5 & 146.8 \\
\hline 1994-1995 & 108 & 8,216 & 41,088 & 26,000 & 157.4 & 157.6 & 148.4 & 142.8 \\
\hline 1993-1994 & 108 & 8,132 & 40,225 & 25,834 & 154.1 & 156.6 & 144.4 & 139.0 \\
\hline 1992-1993 & 108 & 8,212 & 39,265 & 25,000 & 150.4 & 151.5 & 140.5 & 134.9 \\
\hline 1991-1992 & 107 & 8,256 & 38,537 & 24,000 & 147.7 & 145.5 & 136.2 & 131.1 \\
\hline 1990-1991 & 107 & 8,382 & 36,701 & 23,800 & 140.6 & 144.2 & 130.4 & 125.8 \\
\hline 1989-1990 & 107 & 8,253 & 34,629 & 22,000 & 132.7 & 133.3 & 124.4 & 119.3 \\
\hline 1988-1989 & 107 & 8,087 & 32,461 & 20,400 & 124.4 & 123.6 & 118.5 & 113.9 \\
\hline 1987-1988 & 106 & 7,962 & 30,534 & 19,460 & 117.0 & 117.9 & 113.8 & 109.3 \\
\hline 1986-1987 & 105 & 7,718 & 28,941 & 18,250 & 110.9 & 110.6 & 109.5 & 105.5 \\
\hline 1985-1986 & 105 & 7,543 & 27,485 & 17,500 & 105.3 & 106.1 & 107.8 & 103.6 \\
\hline 1984-1985 & 104 & 7,161 & 26,100 & 16,500 & 100.0 & 100.0 & 104.1 & 100.0 \\
\hline
\end{tabular}

*Actual CPI figures have been revised from previous editions based upon changes published by the Bureau of Labor Statistics. These changes are minute, less than 0.3 in all cases.

† Includes medical and law libraries.

‡ Beginning professional salary. 


\section{TABle 3: Salary Trends in US ARL University Libraries}

Salary figures for the current year are displayed in the context of previous years and compared to the changes in the US Consumer Price Index (CPI) to show trends in the purchasing power of median and beginning professional salaries. Salary figures and CPI numbers have been converted to adjusted indexes, using July 1984 as the base. Actual CPI data retrieved from the US Department of Labor, Bureau of Labor Statistics' Consumer Price Index-All Urban Consumers (US All items, 1982-1984=100 - CUUR0000SA0) available online at http://www. bls.gov/data/.

\begin{tabular}{|c|c|c|c|c|c|c|c|c|}
\hline $\begin{array}{l}\text { Fiscal } \\
\text { Year }\end{array}$ & $\begin{array}{l}\text { Number of } \\
\text { Libraries }\end{array}$ & $\begin{array}{l}\text { Total } \\
\text { Staff }\end{array}$ & $\begin{array}{c}\text { Median } \\
\text { Salary }^{\dagger}\end{array}$ & $\begin{array}{c}\text { Median } \\
\text { Salary Change }\end{array}$ & $\begin{array}{c}\text { Median } \\
\text { Salary Index }\end{array}$ & $\begin{array}{l}\text { Actual } \\
\text { CPI* }^{*}\end{array}$ & $\begin{array}{l}\text { Adjusted } \\
\text { CPI }\end{array}$ & $\begin{array}{c}\text { CPI } \\
\text { Change }\end{array}$ \\
\hline 2010-2011 & 99 & 8,925 & $\$ 65,000$ & $1.5 \%$ & 250.5 & 218.0 & 209.8 & $1.2 \%$ \\
\hline 2009-2010 & 99 & 9,116 & 64,069 & 0.6 & 246.9 & 215.4 & 207.3 & -2.0 \\
\hline 2008-2009 & 99 & 9,158 & 63,673 & 3.8 & 245.4 & 219.9 & 211.6 & 5.6 \\
\hline 2007-2008 & 99 & 9,026 & 61,329 & 3.5 & 236.4 & 208.3 & 200.5 & 2.4 \\
\hline 2006-2007 & 99 & 8,866 & 59,280 & 3.7 & 228.5 & 203.5 & 195.9 & 4.1 \\
\hline 2005-2006 & 99 & 8,700 & 57,173 & 2.8 & 220.4 & 195.4 & 188.1 & 3.2 \\
\hline 2004-2005 & 99 & 8,581 & 55,600 & 3.2 & 214.3 & 189.4 & 182.3 & 3.0 \\
\hline 2003-2004 & 100 & 8,581 & 53,859 & 2.0 & 207.6 & 183.9 & 177.0 & 2.1 \\
\hline 2002-2003 & 100 & 8,544 & 52,789 & 1.9 & 203.5 & 180.1 & 173.3 & 1.5 \\
\hline 2001-2002 & 99 & 8,337 & 51,806 & 4.1 & 199.7 & 177.5 & 170.8 & 2.7 \\
\hline 2000-2001 & 99 & 8,127 & 49,753 & 3.7 & 191.8 & 172.8 & 166.3 & 3.7 \\
\hline 1999-2000 & 98 & 7,858 & 48,000 & 4.1 & 185.0 & 166.7 & 160.4 & 2.1 \\
\hline 1998-1999 & 97 & 7,671 & 46,130 & 3.6 & 177.8 & 163.2 & 157.1 & 1.7 \\
\hline 1997-1998 & 97 & 7,682 & 44,544 & 3.4 & 171.7 & 160.5 & 154.5 & 2.2 \\
\hline 1996-1997 & 96 & 7,562 & 43,084 & 3.4 & 166.1 & 157.0 & 151.1 & 3.0 \\
\hline 1995-1996 & 95 & 7,435 & 41,651 & 2.7 & 160.5 & 152.5 & 146.8 & 2.8 \\
\hline 1994-1995 & 95 & 7,401 & 40,573 & 3.4 & 156.4 & 148.4 & 142.8 & 2.8 \\
\hline 1993-1994 & 95 & 7,390 & 39,257 & 3.0 & 151.3 & 144.4 & 139.0 & 2.8 \\
\hline 1992-1993 & 95 & 7,375 & 38,124 & 3.0 & 146.9 & 140.5 & 134.9 & 3.2 \\
\hline 1991-1992 & 94 & 7,408 & 37,009 & 3.5 & 142.6 & 136.2 & 131.1 & 4.4 \\
\hline 1990-1991 & 94 & 7,543 & 35,761 & 5.2 & 137.8 & 130.4 & 125.8 & 4.8 \\
\hline 1989-1990 & 94 & 7,344 & 34,000 & 5.8 & 131.0 & 124.4 & 119.3 & 5.0 \\
\hline 1988-1989 & 94 & 7,252 & 32,149 & 5.4 & 123.9 & 118.5 & 113.9 & 4.1 \\
\hline 1987-1988 & 93 & 7,145 & 30,492 & 5.1 & 117.5 & 113.8 & 109.3 & 3.9 \\
\hline 1986-1987 & 92 & 6,886 & 29,021 & 6.5 & 111.9 & 109.5 & 105.5 & 1.6 \\
\hline 1985-1986 & 91 & 6,707 & 27,249 & 5.0 & 105.0 & 107.8 & 103.6 & 3.6 \\
\hline 1984-1985 & 91 & 6,456 & 25,946 & 6.9 & 100.0 & 104.1 & 100.0 & - \\
\hline
\end{tabular}

*Actual CPI figures have been revised from previous editions based upon changes published by the Bureau of Labor Statistics. These changes are minute, less than 0.3 in all cases.

+ Includes medical and law libraries. 


\section{Table 4: Salary Trends in Canadian ARL University Libraries}

Salary figures for the current year are displayed in the context of previous years. Canadian salaries are presented in both US \$ and Canadian \$ denominations and the annual exchange rate used in the salary surveys is also listed. Canadian salaries are also compared to the changes in the Canadian Consumer Price Index (CPI) to show trends in the purchasing power of median Canadian salaries. CPI number changes are based on July CPI figures. The source for Canadian CPI data is "Table 5: The Consumer Price Index for Canada" published in The Daily, a Statistics Canada publication, available online at http://www.statcan.gc.ca/pub/62-001-x/2011004/t040-eng.htm.

\begin{tabular}{|c|c|c|c|c|c|c|c|c|c|}
\hline $\begin{array}{c}\text { Fiscal } \\
\text { Year }\end{array}$ & $\begin{array}{c}\text { Number } \\
\text { of } \\
\text { Libraries }\end{array}$ & $\begin{array}{l}\text { Total } \\
\text { Staff }\end{array}$ & $\begin{array}{l}\text { Median } \\
\text { Salary } \\
\text { in US } \$^{+}\end{array}$ & $\begin{array}{l}\text { Median } \\
\text { Salary } \\
\text { Change }^{\dagger}\end{array}$ & $\begin{array}{c}\text { Exchange } \\
\text { Rate }\end{array}$ & $\begin{array}{l}\text { Median } \\
\text { Salary } \\
\text { in Can. \$ }\end{array}$ & $\begin{array}{c}\text { Median } \\
\text { Salary } \\
\text { Change }\end{array}$ & $\begin{array}{l}\text { Can. } \\
\text { CPI }^{* *}\end{array}$ & $\begin{array}{c}\text { Can. } \\
\text { CPI } \\
\text { Change* }\end{array}$ \\
\hline 2010-2011 & 16 & 1,112 & $\$ 77,919$ & $12.7 \%$ & 1.0556 & $\$ 82,251$ & $2.0 \%$ & 116.8 & $1.8 \%$ \\
\hline 2009-2010 & 15 & 1,091 & 69,130 & $-11.3 \%$ & 1.1667 & 80,654 & 2.4 & 114.7 & -0.9 \\
\hline 2008-2009 & 14 & 990 & 77,954 & 15.8 & 1.0101 & 78,742 & 3.3 & 115.8 & 3.4 \\
\hline $2007-2008$ & 14 & 957 & 67,331 & 6.7 & 1.1323 & 76,239 & 3.9 & 112.0 & 2.2 \\
\hline 2006-2007 & 14 & 958 & 63,112 & 11.8 & 1.16289 & 73,392 & 4.0 & 109.6 & 2.3 \\
\hline 2005-2006 & 14 & 955 & 56,474 & 7.1 & 1.24971 & 70,576 & -0.3 & 107.1 & 2.0 \\
\hline 2004-2005 & 14 & 906 & 52,707 & 16.3 & 1.34328 & 70,800 & 3.5 & 105.0 & 2.3 \\
\hline 2003-2004 & 14 & 911 & 45,310 & 6.2 & 1.51023 & 68,429 & 2.3 & 102.6 & 2.1 \\
\hline 2002-2003 & 14 & 925 & 42,657 & -0.6 & 1.56878 & 66,919 & 2.6 & 100.5 & 2.1 \\
\hline 2001-2002 & 14 & 861 & 42,928 & -1.1 & 1.51919 & 65,215 & 2.1 & 98.4 & 2.7 \\
\hline 2000-2001 & 13 & 755 & 43,394 & 5.0 & 1.47192 & 63,873 & 2.4 & 95.8 & 2.9 \\
\hline 1999-2000 & 13 & 737 & 41,316 & -3.8 & 1.5103 & 62,400 & 2.4 & 93.1 & 1.9 \\
\hline 1998-1999 & 13 & 729 & 42,963 & -2.7 & 1.4177 & 60,909 & 0.9 & 91.4 & 1.0 \\
\hline 1997-1998 & 13 & 732 & 44,167 & 1.4 & 1.3663 & 60,346 & 1.7 & 90.5 & 1.7 \\
\hline 1996-1997 & 13 & 764 & 43,569 & 0.9 & 1.3613 & 59,310 & -0.4 & 89.0 & 1.3 \\
\hline 1995-1996 & 13 & 796 & 43,173 & -1.7 & 1.3794 & 59,554 & 1.3 & 87.9 & 2.6 \\
\hline 1994-1995 & 13 & 815 & 43,919 & -6.0 & 1.3381 & 58,768 & 0.7 & 85.7 & 0.1 \\
\hline 1993-1994 & 13 & 816 & 46,744 & -4.3 & 1.2488 & 58,374 & 2.9 & 85.6 & 1.7 \\
\hline 1992-1993 & 13 & 837 & 48,820 & 2.7 & 1.1623 & 56,744 & 3.4 & 84.2 & 1.2 \\
\hline 1991-1992 & 13 & 847 & 47,519 & 5.5 & 1.1547 & 54,870 & 3.6 & 83.2 & 6.0 \\
\hline 1990-1991 & 13 & 839 & 45,023 & 15.1 & 1.1759 & 52,942 & 12.5 & 78.5 & 4.1 \\
\hline 1989-1990 & 13 & 853 & 39,117 & 12.3 & 1.2026 & 47,042 & 5.3 & 75.4 & 5.3 \\
\hline 1988-1989 & 13 & 837 & 34,826 & 11.7 & 1.2826 & 44,668 & 5.3 & 71.6 & 3.9 \\
\hline 1987-1988 & 13 & 817 & 31,178 & 10.9 & 1.3602 & 42,408 & 9.1 & 68.9 & 4.6 \\
\hline 1986-1987 & 13 & 831 & 28,123 & -1.9 & 1.3817 & 38,858 & 1.2 & 65.9 & 4.1 \\
\hline 1985-1986 & 13 & 829 & 28,666 & 1.1 & 1.3388 & 38,378 & 7.9 & 63.3 & 4.1 \\
\hline 1984-1985 & 12 & 705 & 28,346 & -0.8 & 1.2548 & 35,569 & 0.8 & 60.8 & 4.1 \\
\hline Average & & & & $3.8 \%$ & & & $3.3 \%$ & & \\
\hline
\end{tabular}

† Includes medical and law libraries.

* Canadian CPI change figures have been revised from previous editions based upon changes published by The Daily (Statistics Canada). These changes were caused by rounding; they are minute and are less than 0.3 in all cases.

** Actual Canadian CPI figures have been added to this table (not available in previous editions). 


\section{ARL NoNUNIVERSITY LIBRARIES}

TABles 5-6 

Table 5: Median and Beginning Professional Salaries in ARL Nonuniversity Libraries

\begin{tabular}{|l|r|rc|cc|}
\hline & No. of & \multicolumn{2}{|c|}{ Median Salaries } & \multicolumn{2}{c|}{ Beginning Salaries } \\
& Staff & FY 2009-2010 & FY 2010-2011 & FY 2009-2010 & FY 2010-2011 \\
\hline Boston Public Library & 167 & $\$ 67,184$ & $\$ 67,267$ & $\$ 40,975$ & $\$ 40,975$ \\
Canada Institute for Scientific & 82 & 67,639 & 73,649 & 44,446 & 53,155 \\
and Technical Information * & & & & & 33,878 \\
Center for Research Libraries & 33 & 50,799 & 52,179 & 33,878 & 50,640 \\
Library \& Archives Canada * & 99 & 56,601 & 63,497 & 45,141 & 51,630 \\
Library of Congress & 2,678 & 101,416 & 103,872 & 50,408 & 51,630 \\
National Agricultural Library ${ }^{\dagger}$ & 87 & 82,845 & 84,855 & 50,408 & 42,209 \\
National Library of Medicine & 208 & 90,154 & 92,341 & 50,408 & 42,638 \\
New York Public Library & 244 & 63,696 & 61,438 & 42,638 & 53,366 \\
New York State Library & 54 & 64,305 & 68,637 & 49,968 & 51,630 \\
Smithsonian Library & 57 & 85,281 & 87,350 & 52,089 & \\
\hline
\end{tabular}

* Canadian salaries are expressed in US dollars.

t See footnotes. 


\section{Table 6: Salary Trends in ARL NonUniversity Libraries}

Salary figures for the current year are displayed in the context of the previous years and compared to the changes in the Consumer Price Index (CPI) to show trends in the purchasing power of median and beginning professional salaries. Salary figures and CPI numbers have been converted to adjusted indexes, using July 1984 as the base. Actual CPI data retrieved from the US Department of Labor, Bureau of Labor Statistics' Consumer Price Index-All Urban Consumers (US All items, 1982-1984=100 - CUUR0000SA0) available online at http://www. bls.gov/data/.

Note: Canadian salaries are expressed in US dollars.

\begin{tabular}{|c|c|c|c|c|c|c|c|c|}
\hline $\begin{array}{l}\text { Fiscal } \\
\text { Year }\end{array}$ & $\begin{array}{l}\text { Number of } \\
\text { Libraries }\end{array}$ & Total Staff & $\begin{array}{l}\text { Median } \\
\text { Salary }\end{array}$ & $\begin{array}{c}\text { BPS }^{+} \\
\text {Median }\end{array}$ & $\begin{array}{c}\text { Median } \\
\text { Salary Index }\end{array}$ & $\begin{array}{l}\text { BPS }^{+} \\
\text {Index }\end{array}$ & $\begin{array}{l}\text { Actual } \\
\text { CPI }\end{array}$ & $\begin{array}{l}\text { Adjusted } \\
\text { CPI }\end{array}$ \\
\hline 2010-2011 & 10 & 3,709 & $\$ 95,020$ & $\$ 51,135$ & 280.5 & 309.8 & 218.0 & 209.8 \\
\hline 2009-2010 & 10 & 3,811 & 85,229 & 47,554 & 251.6 & 288.1 & 215.4 & 207.3 \\
\hline 2008-2009 & 10 & 3,748 & 85,320 & 48,108 & 251.8 & 291.4 & 219.9 & 211.6 \\
\hline 2007-2008 & 10 & 3,797 & 80,261 & 44,359 & 236.9 & 268.7 & 208.3 & 200.5 \\
\hline 2006-2007 & 10 & 3,832 & 80,124 & 42,765 & 236.5 & 259.1 & 203.5 & 195.9 \\
\hline 2005-2006 & 10 & 3,921 & 76,083 & 38,673 & 224.6 & 234.3 & 195.4 & 188.1 \\
\hline 2004-2005 & 10 & 3,946 & 74,022 & 34,764 & 218.5 & 210.6 & 189.4 & 182.3 \\
\hline 2003-2004 & 10 & 3,877 & 70,020 & 34,739 & 206.8 & 210.4 & 183.9 & 177.0 \\
\hline 2002-2003 & 10 & 3,804 & 65,289 & 34,739 & 192.7 & 210.4 & 180.1 & 173.3 \\
\hline 2001-2002 & 10 & 3,717 & 65,025 & 34,389 & 191.9 & 208.3 & 177.5 & 170.8 \\
\hline 2000-2001 & 10 & 3,731 & 62,521 & 31,774 & 184.5 & 192.5 & 172.8 & 166.3 \\
\hline 1999-2000 & 10 & 3,737 & 59,916 & 30,849 & 176.8 & 186.9 & 166.7 & 160.4 \\
\hline 1998-1999 & 11 & 3,819 & 56,000 & 29,877 & 165.3 & 181.0 & 163.2 & 157.1 \\
\hline 1997-1998 & 11 & 3,779 & 55,055 & 28,724 & 162.5 & 174.0 & 160.5 & 154.5 \\
\hline 1996-1997 & 11 & 3,799 & 51,150 & 28,380 & 151.0 & 172.0 & 157.0 & 151.1 \\
\hline 1995-1996 & 11 & 3,915 & 49,149 & 28,162 & 145.1 & 170.7 & 152.5 & 146.8 \\
\hline 1994-1995 & 11 & 3,837 & 47,997 & 27,813 & 141.7 & 168.6 & 148.4 & 142.8 \\
\hline 1993-1994 & 11 & 4,003 & 44,949 & 26,806 & 132.7 & 162.5 & 144.4 & 139.0 \\
\hline 1992-1993 & 11 & 4,172 & 43,876 & 23,500 & 129.6 & 142.4 & 140.2 & 134.9 \\
\hline 1991-1992 & 11 & 2,906 & 42,455 & 23,500 & 125.4 & 142.4 & 136.2 & 131.1 \\
\hline 1990-1991 & 12 & 1,363 & 36,013 & 20,800 & 106.3 & 126.1 & 130.7 & 125.8 \\
\hline 1989-1990 & 11 & 3,767 & 40,106 & 20,195 & 118.4 & 122.4 & 124.0 & 119.3 \\
\hline 1988-1989 & 11 & 3,781 & 37,544 & 19,100 & 110.9 & 115.8 & 118.3 & 113.9 \\
\hline 1987-1988 & 11 & 3,765 & 36,250 & 18,405 & 107.0 & 111.5 & 113.6 & 109.3 \\
\hline 1986-1987 & 10 & 2,790 & 33,020 & 17,912 & 97.5 & 108.6 & 109.6 & 105.5 \\
\hline 1985-1986 & 12 & 3,874 & 33,720 & 17,308 & 99.6 & 104.9 & 107.6 & 103.6 \\
\hline 1984-1985 & 11 & 3,840 & 33,863 & 16,500 & 100.0 & 100.0 & 103.9 & 100.0 \\
\hline
\end{tabular}




\section{ARL UnIVERSITY LibRARIES}

TABles 7-25 
Table 7: Filled Positions; Average, Median, and Beginning Salaries; and Average Years of EXPERIENCE IN ARL UNIVERSITY LIBRARIES, FY 2010-2011

\begin{tabular}{|c|c|c|c|c|c|c|c|c|}
\hline \multirow[b]{2}{*}{ Institution } & \multirow{2}{*}{$\begin{array}{c}\text { FILlED } \\
\text { PosITIONS } \\
\text { FY } 2011\end{array}$} & \multicolumn{2}{|c|}{ Average Salaries } & \multicolumn{2}{|c|}{ Median SALARIES } & \multicolumn{2}{|c|}{ BeginNing SAlaries } & \multirow{2}{*}{$\begin{array}{l}\text { Average } \\
\text { YRs. EXP } \\
\text { FY } 2011\end{array}$} \\
\hline & & FY 2010 & FY 2011 & FY 2010 & FY 2011 & FY 2010 & FY 2011 & \\
\hline Alabama $^{\ddagger}$ & 62 & $\$ 58,272$ & $\$ 58,760$ & $\$ 52,204$ & $\$ 55,188$ & $\$ 42,000$ & $\$ 42,000$ & 15.0 \\
\hline Alberta $^{+\ddagger}$ & 72 & 84,172 & 96,215 & 89,319 & 96,759 & 43,398 & 52,833 & 15.7 \\
\hline Arizona $^{\ddagger}$ & 55 & 65,897 & 65,351 & 59,915 & 59,118 & 48,605 & 50,857 & 18.0 \\
\hline Arizona State ${ }^{\ddagger}$ & 56 & 66,091 & 64,744 & 65,071 & 65,039 & 43,000 & 43,000 & 19.9 \\
\hline Auburn ${ }^{\ddagger}$ & 44 & 58,382 & 57,728 & 53,705 & 52,330 & 44,720 & 44,720 & 15.1 \\
\hline Boston University ${ }^{\ddagger}$ & 56 & 57,615 & 59,680 & 56,600 & 57,200 & 43,700 & 33,000 & 17.5 \\
\hline Boston College ${ }^{\ddagger}$ & 55 & 69,739 & 70,871 & 67,280 & 69,560 & 42,300 & 43,350 & 19.7 \\
\hline Brigham Young & 109 & 66,005 & 67,295 & 63,950 & 66,000 & 51,000 & 52,020 & 18.9 \\
\hline British Columbia ${ }^{\ddagger \ddagger}$ & 72 & 73,023 & 85,029 & 70,005 & 83,131 & 47,429 & 52,420 & 17.7 \\
\hline Brown $^{\ddagger}$ & 62 & 65,880 & 66,368 & 61,549 & 62,972 & 39,500 & 40,500 & 18.8 \\
\hline Calgary $^{\text {t\# }}$ & 53 & 81,275 & 91,776 & 76,749 & 88,732 & 49,713 & 54,945 & 18.7 \\
\hline California, Berkeley ${ }^{\ddagger}$ & 101 & 83,853 & 85,329 & 82,524 & 82,524 & 47,087 & 46,164 & 18.0 \\
\hline California, Davis ${ }^{\ddagger}$ & 39 & 81,135 & 84,334 & 88,488 & 88,488 & 46,164 & 46,164 & 21.8 \\
\hline California, Irvine & 51 & 76,645 & 77,825 & 75,708 & 75,708 & 46,164 & 46,144 & 16.8 \\
\hline California, Los Angeles ₹ & 136 & 78,189 & 79,113 & 75,708 & 75,708 & 46,164 & 46,164 & 16.3 \\
\hline California, Riverside ${ }^{\ddagger}$ & 43 & 77,661 & 79,762 & 75,708 & 79,116 & 47,087 & 47,087 & 21.5 \\
\hline California, San Diego ${ }^{\ddagger}$ & 84 & 77,158 & 77,064 & 75,708 & 75,708 & 46,164 & 46,164 & 17.4 \\
\hline California, Santa Barbara ${ }^{\ddagger}$ & 55 & 71,378 & 74,431 & 68,892 & 74,104 & 46,164 & 46,164 & 17.8 \\
\hline Case Western Reserve ${ }^{\ddagger}$ & 43 & 58,788 & 58,886 & 55,227 & 55,434 & 35,000 & 35,000 & 15.9 \\
\hline Chicago $^{\ddagger}$ & 71 & 76,254 & 76,692 & 72,329 & 73,130 & 48,204 & 50,151 & 20.8 \\
\hline Cincinnati ${ }^{\ddagger}$ & 58 & 65,445 & 68,451 & 61,879 & 66,260 & 40,000 & 42,000 & 19.5 \\
\hline Colorado $^{\ddagger}$ & 45 & 67,523 & 64,595 & 64,573 & 61,262 & 44,000 & 44,000 & 14.2 \\
\hline Colorado State & 37 & 68,311 & 67,844 & 65,300 & 64,700 & 45,000 & 55,000 & 18.0 \\
\hline Columbia $^{\ddagger}$ & 204 & 72,939 & 74,360 & 64,385 & 65,280 & 51,500 & 52,000 & 15.8 \\
\hline Connecticut ${ }^{\ddagger}$ & 60 & 82,083 & 81,341 & 78,079 & 79,309 & 48,000 & 50,000 & 17.8 \\
\hline Cornell $^{\ddagger}$ & 102 & 71,548 & 74,377 & 64,239 & 66,873 & 47,000 & 48,000 & 17.1 \\
\hline Dartmouth $^{\ddagger}$ & 47 & 72,208 & 70,594 & 67,933 & 67,159 & 45,500 & 45,500 & 17.6 \\
\hline Delaware & 59 & 75,703 & 76,673 & 73,353 & 72,868 & 43,600 & 43,600 & 18.2 \\
\hline Duke ${ }^{\ddagger}$ & 117 & 64,870 & 65,526 & 60,000 & 60,000 & 45,000 & 45,000 & 15.0 \\
\hline Emory ${ }^{\ddagger}$ & 67 & 68,747 & 69,761 & 63,068 & 63,198 & 42,000 & 47,750 & 15.0 \\
\hline Florida $^{\ddagger}$ & 68 & 60,450 & 62,229 & 56,388 & 58,073 & 42,000 & 42,000 & 16.3 \\
\hline Florida State $^{\ddagger}$ & 41 & 54,858 & 56,313 & 49,805 & 52,265 & 42,000 & 42,000 & 12.5 \\
\hline George Washington ₹ & 41 & 74,353 & 76,999 & 67,143 & 72,277 & 47,000 & 47,000 & 15.9 \\
\hline Georgetown ${ }^{\ddagger}$ & 53 & 69,227 & 70,338 & 63,538 & 63,965 & 45,000 & 45,000 & 21.6 \\
\hline Georgia $^{\ddagger}$ & 69 & 56,544 & 56,057 & 50,000 & 49,795 & 38,000 & 38,000 & 16.5 \\
\hline Georgia Tech ${ }^{\ddagger}$ & 44 & 63,526 & 63,534 & 58,016 & 58,016 & 44,000 & 44,000 & 14.8 \\
\hline Guelph ${ }^{+\ddagger}$ & 50 & 69,113 & 80,588 & 66,902 & 76,872 & 51,169 & 58,392 & 18.1 \\
\hline Harvard $^{\ddagger}$ & 408 & 77,319 & 79,111 & 70,720 & 72,134 & 48,800 & 53,093 & 15.4 \\
\hline Hawaii ${ }^{\ddagger}$ & 67 & 66,721 & 63,037 & 66,225 & 64,692 & 42,660 & 35,000 & 18.2 \\
\hline Houston ${ }^{\ddagger}$ & 41 & 61,669 & 62,188 & 56,563 & 54,628 & 43,000 & 44,000 & 15.0 \\
\hline Howard ${ }^{\ddagger}$ & 13 & 43,547 & 56,590 & 43,010 & 50,223 & 38,000 & 34,627 & 22.5 \\
\hline Illinois, Chicago ${ }^{\ddagger}$ & 43 & 62,208 & 63,420 & 56,555 & 57,730 & 47,000 & 47,000 & 17.0 \\
\hline
\end{tabular}

28 · ARL Annual Salary Survey 2010-2011 
Table 7: Filled Positions; Average, Median, and Beginning Salaries; and Average Years of EXPERIENCE IN ARL UNIVERSITY LIBRARIES, FY 2010-2011

\begin{tabular}{|c|c|c|c|c|c|c|c|c|}
\hline \multirow[b]{2}{*}{ Institution } & \multirow{2}{*}{$\begin{array}{c}\text { FILled } \\
\text { Positions } \\
\text { FY } 2011 \\
\end{array}$} & \multicolumn{2}{|c|}{ Average Salaries } & \multicolumn{2}{|c|}{ Median Salaries } & \multicolumn{2}{|c|}{ Beginning Salaries } & \multirow{2}{*}{$\begin{array}{l}\text { AvERAG } \\
\text { YRS. EXP } \\
\text { FY } 2011\end{array}$} \\
\hline & & FY 2010 & FY 2011 & FY 2010 & FY 2011 & FY 2010 & FY 2011 & \\
\hline Illinois, Urbana ${ }^{\ddagger}$ & 127 & 66,755 & 69,964 & 61,645 & 65,129 & 46,000 & 50,000 & 16.2 \\
\hline Indiana ${ }^{\ddagger}$ & 78 & 63,870 & 63,918 & 59,331 & 59,331 & 40,400 & 40,400 & 19.5 \\
\hline Iowa ${ }^{\ddagger}$ & 65 & 62,529 & 64,501 & 55,573 & 57,840 & 41,000 & 41,000 & 18.8 \\
\hline Iowa State ${ }^{\ddagger}$ & 49 & 63,217 & 65,451 & 61,664 & 63,105 & 43,000 & 44,000 & 20.5 \\
\hline Johns Hopkins ${ }^{\ddagger}$ & 83 & 68,926 & 69,299 & 65,690 & 65,353 & 50,026 & 51,027 & 16.7 \\
\hline Kansas $^{\ddagger}$ & 85 & 60,877 & 62,232 & 54,806 & 57,417 & 51,000 & 43,000 & 16.1 \\
\hline Kent State ${ }^{\ddagger}$ & 63 & 63,997 & 66,624 & 60,301 & 64,520 & 55,367 & 57,078 & 16.8 \\
\hline Kentucky ${ }^{\ddagger}$ & 64 & 60,966 & 60,762 & 59,839 & 59,984 & 41,000 & 41,000 & 21.7 \\
\hline Laval $^{+\ddagger}$ & 65 & 61,963 & 67,439 & 62,474 & 69,944 & 42,468 & 46,937 & 13.1 \\
\hline Louisiana State & 49 & 50,821 & 51,100 & 46,462 & 46,901 & 38,000 & 38,000 & 15.1 \\
\hline Louisville ${ }^{\ddagger}$ & 31 & 59,483 & 59,638 & 55,884 & 53,031 & 37,000 & 37,000 & 17.3 \\
\hline McGill ${ }^{\ddagger}$ & 64 & 63,797 & 71,450 & 57,834 & 64,865 & 42,856 & 47,366 & 15.7 \\
\hline McMaster $^{\dagger \neq}$ & 45 & 61,608 & 69,041 & 62,508 & 62,118 & 40,128 & 45,945 & 17.6 \\
\hline Manitoba $^{\dagger \ddagger}$ & 40 & 81,855 & 91,679 & 85,771 & 95,343 & 41,845 & 46,249 & 22.9 \\
\hline Maryland & 72 & 68,874 & 69,981 & 66,603 & 69,035 & 40,000 & 40,000 & 21.6 \\
\hline Massachusetts ${ }^{*}$ & 57 & 69,882 & 72,957 & 72,263 & 73,553 & 42,155 & 42,155 & 17.4 \\
\hline $\mathrm{MIT}^{\ddagger}$ & 93 & 74,430 & 77,065 & 70,959 & 73,326 & 51,750 & 52,000 & 16.6 \\
\hline Miami ${ }^{\ddagger}$ & 52 & 68,278 & 69,503 & 61,728 & 65,000 & 45,000 & 45,000 & 15.6 \\
\hline Michigan ${ }^{\ddagger}$ & 169 & 67,767 & 71,354 & 63,717 & 65,884 & 42,000 & 42,000 & 17.4 \\
\hline Michigan State ${ }^{\ddagger}$ & 65 & 68,894 & 70,979 & 65,850 & 67,373 & 47,000 & 47,500 & 17.6 \\
\hline Minnesota & 107 & 66,794 & 68,210 & 63,557 & 65,785 & 42,000 & 43,000 & 16.9 \\
\hline Missouri ${ }^{\ddagger}$ & 36 & 59,914 & 59,710 & 55,964 & 57,127 & 40,000 & 40,000 & 21.0 \\
\hline Montreal ${ }^{+\ddagger}$ & 89 & 62,740 & 70,501 & 59,349 & 65,596 & 36,968 & 43,497 & 15.3 \\
\hline Nebraska ${ }^{\ddagger}$ & 46 & 65,057 & 64,928 & 57,472 & 57,453 & 50,000 & 50,000 & 20.0 \\
\hline New Mexico ${ }^{\ddagger}$ & 44 & 72,938 & 71,159 & 68,853 & 67,913 & 40,000 & 40,000 & 19.5 \\
\hline New York University ${ }^{\ddagger}$ & 84 & 79,018 & 78,859 & 69,989 & 71,616 & 52,000 & 55,000 & 15.8 \\
\hline North Carolina & 96 & 67,637 & 65,091 & 63,712 & 62,300 & 44,000 & 44,000 & 17.3 \\
\hline North Carolina State ${ }^{\ddagger}$ & 91 & 73,019 & 72,411 & 65,000 & 65,000 & 50,000 & 52,000 & 11.1 \\
\hline Northwestern ${ }^{\ddagger}$ & 89 & 67,873 & 68,146 & 63,429 & 62,995 & 43,000 & 44,000 & 16.3 \\
\hline Notre Dame ${ }^{\ddagger}$ & 58 & 69,492 & 71,751 & 66,508 & 67,915 & 41,200 & 44,000 & 19.5 \\
\hline Ohio University $^{\ddagger}$ & 45 & 55,522 & 55,590 & 48,787 & 49,275 & 41,000 & 41,500 & 13.6 \\
\hline Ohio State ${ }^{\ddagger}$ & 124 & 59,476 & 57,846 & 54,450 & 53,292 & 46,000 & 46,000 & 14.2 \\
\hline Oklahoma & 42 & 55,853 & 55,299 & 53,742 & 53,477 & 42,000 & 42,000 & 14.6 \\
\hline Oklahoma State ${ }^{\ddagger}$ & 64 & 56,580 & 56,678 & 52,657 & 51,865 & 38,000 & 38,000 & 17.5 \\
\hline Oregon ${ }^{\ddagger}$ & 54 & 59,782 & 61,130 & 55,973 & 57,839 & 40,000 & 40,000 & 17.3 \\
\hline Ottawa $a^{\dagger \ddagger^{*}}$ & 34 & $\mathrm{~N} / \mathrm{A}$ & 87,360 & $\mathrm{~N} / \mathrm{A}$ & 85,122 & $\mathrm{~N} / \mathrm{A}$ & 47,538 & 18.9 \\
\hline Pennsylvania ${ }^{\ddagger}$ & 104 & 65,997 & 66,632 & 61,850 & 63,148 & 43,500 & 41,000 & 15.6 \\
\hline Pennsylvania State ${ }^{\ddagger}$ & 143 & 67,897 & 69,465 & 64,224 & 66,258 & 42,436 & 43,709 & 19.1 \\
\hline Pittsburgh ${ }^{\ddagger}$ & 65 & 66,551 & 69,211 & 60,711 & 62,897 & 34,000 & 34,000 & 19.8 \\
\hline Princeton ${ }^{\ddagger}$ & 112 & 79,015 & 82,454 & 73,799 & 76,100 & 63,200 & 63,200 & 20.9 \\
\hline Purdue ${ }^{\ddagger}$ & 63 & 65,573 & 65,562 & 60,513 & 61,006 & 47,000 & 47,000 & 19.3 \\
\hline Queen's ${ }^{+}$ & 32 & 80,504 & 96,157 & 83,438 & 99,005 & 44,227 & 50,446 & 21.3 \\
\hline
\end{tabular}


Table 7: Filled Positions; Average, Median, and Beginning Salaries; and Average Years of EXPERIENCE IN ARL UNIVERSITY LIBRARIES, FY 2010-2011

\begin{tabular}{|c|c|c|c|c|c|c|c|c|}
\hline \multirow[b]{2}{*}{ Institution } & \multirow{2}{*}{$\begin{array}{c}\text { FILLED } \\
\text { Positions } \\
\text { FY } 2011\end{array}$} & \multicolumn{2}{|c|}{ Average Salaries } & \multicolumn{2}{|c|}{ Median Salaries } & \multicolumn{2}{|c|}{ Beginning Salaries } & \multirow{2}{*}{$\begin{array}{l}\text { AvERAGE } \\
\text { YRS. EXP. } \\
\text { FY } 2011\end{array}$} \\
\hline & & FY 2010 & FY 2011 & FY 2010 & FY 2011 & FY 2010 & FY 2011 & \\
\hline Rice & 59 & 62,215 & 65,202 & 55,833 & 60,300 & 37,200 & 38,700 & 15.4 \\
\hline Rochester ${ }^{\ddagger}$ & 67 & 55,837 & 59,814 & 53,000 & 56,059 & 38,168 & 38,983 & 16.4 \\
\hline Rutgers $^{\neq}$ & 86 & 89,058 & 91,176 & 91,751 & 91,751 & 49,286 & 50,765 & 22.5 \\
\hline Saskatchewan ${ }^{+\neq}$ & 46 & 76,307 & 85,160 & 78,850 & 81,582 & 45,197 & 52,202 & 16.0 \\
\hline South Carolina ${ }^{\ddagger}$ & 48 & 53,658 & 52,032 & 49,838 & 48,627 & 34,000 & 38,000 & 16.9 \\
\hline Southern California ${ }^{\ddagger}$ & 105 & 74,479 & 76,057 & 67,650 & 69,761 & 48,500 & 48,500 & 19.6 \\
\hline Southern Illinois & 36 & 58,264 & 59,157 & 52,536 & 56,912 & 44,000 & 44,000 & 14.9 \\
\hline SUNY Albany ${ }^{\ddagger}$ & 62 & 60,964 & 64,024 & 58,822 & 62,729 & 39,000 & 39,350 & 17.3 \\
\hline SUNY Buffalo ${ }^{\ddagger}$ & 72 & 72,916 & 75,265 & 69,048 & 70,554 & 45,000 & 47,000 & 19.9 \\
\hline SUNY Stony Brook ${ }^{\ddagger}$ & 24 & 79,837 & 87,447 & 75,457 & 79,599 & 42,000 & 43,000 & 22.3 \\
\hline Syracuse ${ }^{\ddagger}$ & 54 & 65,847 & 67,893 & 60,648 & 61,390 & None & 38,000 & 19.3 \\
\hline Temple ${ }^{\ddagger}$ & 39 & 66,849 & 69,082 & 58,140 & 60,048 & 44,044 & 44,004 & 19.7 \\
\hline Tennessee $^{\ddagger}$ & 40 & 69,380 & 68,677 & 66,307 & 67,268 & 44,000 & 44,000 & 18.9 \\
\hline Texas $^{\ddagger}$ & 121 & 66,882 & 66,964 & 59,260 & 59,304 & 46,000 & 45,000 & 17.1 \\
\hline Texas A\&M ${ }^{\ddagger}$ & 113 & 62,441 & 64,966 & 55,704 & 59,055 & 47,500 & 48,500 & 15.0 \\
\hline Texas Tech ${ }^{\ddagger}$ & 68 & 60,690 & 58,352 & 57,135 & 54,163 & 45,000 & 45,000 & 12.6 \\
\hline Toronto $^{+}$ & 141 & 78,211 & 85,574 & 75,194 & 82,254 & 44,484 & 49,451 & 15.6 \\
\hline Tulane & 35 & 60,534 & 62,835 & 56,325 & 59,492 & 40,000 & 40,000 & 17.9 \\
\hline Utah & 58 & 61,470 & 61,061 & 56,312 & 55,375 & 44,000 & 45,000 & 19.2 \\
\hline Vanderbilt $^{\ddagger}$ & 62 & 58,991 & 59,927 & 54,655 & 55,945 & 40,500 & 41,000 & 18.0 \\
\hline Virginia $\neq$ & 72 & 67,988 & 70,351 & 60,000 & 64,600 & 44,000 & 44,000 & 17.7 \\
\hline Virginia Tech ₹ & 38 & 63,148 & 63,308 & 58,540 & 58,540 & 40,000 & 40,000 & 16.8 \\
\hline Washington ${ }^{\ddagger}$ & 115 & 66,476 & 65,466 & 59,934 & 58,752 & 42,600 & 42,600 & 19.2 \\
\hline Washington State ${ }^{\ddagger}$ & 42 & 62,755 & 63,512 & 58,172 & 58,172 & 38,500 & 38,500 & 19.6 \\
\hline Washington U.-St. Louis ${ }^{\ddagger}$ & 62 & 59,037 & 60,832 & 53,740 & 55,489 & 40,000 & 40,000 & 17.2 \\
\hline Waterloo $^{+\neq}$ & 36 & 70,230 & 76,645 & 70,260 & 75,974 & 44,402 & 49,110 & 17.4 \\
\hline Wayne State ${ }^{\ddagger}$ & 73 & 61,261 & 60,697 & 54,705 & 55,396 & 40,500 & 41,000 & 13.4 \\
\hline Western Ontario $^{+\neq}$ & 65 & 59,073 & 68,581 & 55,725 & 66,961 & 40,610 & 47,836 & 13.4 \\
\hline Wisconsin $^{\ddagger}$ & 155 & 61,856 & 61,068 & 58,654 & 57,692 & 40,526 & 40,526 & 18.1 \\
\hline Yale ${ }^{\ddagger}$ & 191 & 81,088 & 80,642 & 76,300 & 76,365 & 50,500 & 50,500 & 19.0 \\
\hline York $^{+\neq}$ & 53 & 82,297 & 99,770 & 77,321 & 96,446 & 41,999 & 46,419 & 17.6 \\
\hline
\end{tabular}


Page Intentionally Left Blank.

ARL University Libraries · 31 
Table 8: Beginning Professional Salaries in ARL University Libraries

Rank Order Table, FY 2009-2010

\begin{tabular}{|c|c|c|c|c|c|}
\hline Rank & Institution & Salary & Rank & Institution & Salary \\
\hline $\begin{array}{r}1 \\
2 \\
3 \\
4 \\
5 \\
6 \\
7 \\
7 \\
9 \\
10\end{array}$ & $\begin{array}{l}\text { Princeton } \\
\text { Kent State } \\
\text { New York } \\
\text { MIT } \\
\text { Columbia } \\
\text { Guelph } \\
\text { Brigham Young } \\
\text { Kansas } \\
\text { Yale } \\
\text { Johns Hopkins }\end{array}$ & $\begin{array}{l}63,200 \\
55,367 \\
52,000 \\
51,750 \\
51,500 \\
51,169 \\
51,000 \\
51,000 \\
50,500 \\
50,026\end{array}$ & $\begin{array}{l}58 \\
59 \\
60 \\
61 \\
61 \\
61 \\
61 \\
65 \\
66 \\
67\end{array}$ & $\begin{array}{l}\text { Delaware } \\
\text { Pennsylvania } \\
\text { Alberta } \\
\text { Arizona State } \\
\text { Houston } \\
\text { IowaState } \\
\text { Northwestern } \\
\text { McGill } \\
\text { Hawaii } \\
\text { Washington }\end{array}$ & $\begin{array}{l}43,600 \\
43,500 \\
43,398 \\
43,000 \\
43,000 \\
43,000 \\
43,000 \\
42,856 \\
42,660 \\
42,600\end{array}$ \\
\hline $\begin{array}{l}11 \\
11 \\
13 \\
14 \\
15 \\
16 \\
17 \\
18 \\
19 \\
20\end{array}$ & $\begin{array}{l}\text { Nebraska } \\
\text { North Carolina State } \\
\text { Calgary } \\
\text { Rutgers } \\
\text { Harvard } \\
\text { Arizona } \\
\text { Southern California } \\
\text { Chicago } \\
\text { Connecticut } \\
\text { Texas A\&M }\end{array}$ & $\begin{array}{l}50,000 \\
50,000 \\
49,713 \\
49,286 \\
48,800 \\
48,605 \\
48,500 \\
48,204 \\
48,000 \\
47,500\end{array}$ & $\begin{array}{l}68 \\
69 \\
70 \\
71 \\
72 \\
72 \\
72 \\
72 \\
72 \\
72\end{array}$ & $\begin{array}{l}\text { Laval } \\
\text { Pennsylvania State } \\
\text { Boston College } \\
\text { Massachusetts } \\
\text { Alabama } \\
\text { Emory } \\
\text { Florida } \\
\text { Florida State } \\
\text { Michigan } \\
\text { Minnesota }\end{array}$ & $\begin{array}{l}42,468 \\
42,436 \\
42,300 \\
42,155 \\
42,000 \\
42,000 \\
42,000 \\
42,000 \\
42,000 \\
42,000\end{array}$ \\
\hline $\begin{array}{l}21 \\
22 \\
22 \\
24 \\
24 \\
24 \\
24 \\
24 \\
29 \\
29\end{array}$ & $\begin{array}{l}\text { British Columbia } \\
\text { Calif. Berkeley } \\
\text { Calif. Riverside } \\
\text { Cornell } \\
\text { George Washington } \\
\text { Illinois, Chicago } \\
\text { Michigan State } \\
\text { Purdue } \\
\text { Calif. Davis } \\
\text { Calif. Irvine }\end{array}$ & $\begin{array}{l}47,429 \\
47,087 \\
47,087 \\
47,000 \\
47,000 \\
47,000 \\
47,000 \\
47,000 \\
46,164 \\
46,164\end{array}$ & $\begin{array}{l}72 \\
72 \\
80 \\
81 \\
82 \\
83 \\
83 \\
83 \\
86 \\
87\end{array}$ & $\begin{array}{l}\text { Oklahoma } \\
\text { SUNY Stony Brook } \\
\text { York } \\
\text { Manitoba } \\
\text { Notre Dame } \\
\text { lowa } \\
\text { Kentucky } \\
\text { Ohio } \\
\text { Western Ontario } \\
\text { Wisconsin }\end{array}$ & $\begin{array}{l}42,000 \\
42,000 \\
41,999 \\
41,845 \\
41,200 \\
41,000 \\
41,000 \\
41,000 \\
40,610 \\
40,526\end{array}$ \\
\hline $\begin{array}{l}29 \\
29 \\
29 \\
34 \\
34 \\
34 \\
37 \\
38 \\
39 \\
39\end{array}$ & $\begin{array}{l}\text { Calif. Los Angeles } \\
\text { Calif. San Diego } \\
\text { Calif. Santa Barbara } \\
\text { Illinois, Urbana } \\
\text { Ohio State } \\
\text { Texas } \\
\text { Dartmouth } \\
\text { Saskatchewan } \\
\text { Colorado State } \\
\text { Duke }\end{array}$ & $\begin{array}{l}46,164 \\
46,164 \\
46,164 \\
46,000 \\
46,000 \\
46,000 \\
45,500 \\
45,197 \\
45,000 \\
45,000\end{array}$ & $\begin{array}{l}88 \\
88 \\
90 \\
91 \\
92 \\
92 \\
92 \\
92 \\
92 \\
92\end{array}$ & $\begin{array}{l}\text { Vanderbilt } \\
\text { Wayne State } \\
\text { Indiana } \\
\text { McMaster } \\
\text { Cincinnati } \\
\text { Maryland } \\
\text { Missouri } \\
\text { New Mexico } \\
\text { Oregon } \\
\text { Tulane }\end{array}$ & $\begin{array}{l}40,500 \\
40,500 \\
40,400 \\
40,128 \\
40,000 \\
40,000 \\
40,000 \\
40,000 \\
40,000 \\
40,000\end{array}$ \\
\hline $\begin{array}{l}39 \\
39 \\
39 \\
39 \\
45 \\
46 \\
47 \\
48 \\
49 \\
50\end{array}$ & $\begin{array}{l}\text { Georgetown } \\
\text { Miami } \\
\text { SUNY Buffalo } \\
\text { Texas Tech } \\
\text { Auburn } \\
\text { Toronto } \\
\text { Waterloo } \\
\text { Queen's } \\
\text { Temple } \\
\text { Colorado }\end{array}$ & $\begin{array}{l}45,000 \\
45,000 \\
45,000 \\
45,000 \\
44,720 \\
44,484 \\
44,402 \\
44,227 \\
44,044 \\
44,000\end{array}$ & $\begin{array}{r}92 \\
92 \\
100 \\
101 \\
102 \\
103 \\
104 \\
104 \\
104 \\
104\end{array}$ & $\begin{array}{l}\text { Virginia Tech } \\
\text { Washington-St. Louis } \\
\text { Brown } \\
\text { SUNY Albany } \\
\text { Washington State } \\
\text { Rochester } \\
\text { Georgia } \\
\text { Howard } \\
\text { Louisiana State } \\
\text { Oklahoma State }\end{array}$ & $\begin{array}{l}40,000 \\
40,000 \\
39,500 \\
39,000 \\
38,500 \\
38,168 \\
38,000 \\
38,000 \\
38,000 \\
38,000\end{array}$ \\
\hline $\begin{array}{l}50 \\
50 \\
50 \\
50 \\
50 \\
50 \\
57\end{array}$ & $\begin{array}{l}\text { Georgia Tech } \\
\text { North Carolina } \\
\text { Southern Illinois } \\
\text { Tennessee } \\
\text { Utah } \\
\text { Virginia } \\
\text { Boston University }\end{array}$ & $\begin{array}{l}44,000 \\
44,000 \\
44,000 \\
44,000 \\
44,000 \\
44,000 \\
43,700\end{array}$ & $\begin{array}{r}108 \\
109 \\
110 \\
111 \\
112 \\
112 \\
\text { N/A }\end{array}$ & $\begin{array}{l}\text { Rice } \\
\text { Louisville } \\
\text { Montreal } \\
\text { Case Western Reserve } \\
\text { Pittsburgh } \\
\text { South Carolina } \\
\text { Syracuse }\end{array}$ & $\begin{array}{r}37,200 \\
37,000 \\
36,968 \\
35,000 \\
34,000 \\
34,000 \\
\text { None }\end{array}$ \\
\hline
\end{tabular}

Reprinted from ARL Annual Salary Survey 2009-2010. Beginning salary figures represent officially designated base, not necessarily salaries of actual incumbents.

Excludes medical and law libraries. See Tables 36 and 43 for comparable figures for medical and law libraries.

Canadian salaries are expressed in US dollars. 
Table 9: Beginning Professional Salaries in ARL University Libraries

Rank ORder Table, FY 2010-2011

\begin{tabular}{|c|c|c|c|c|c|}
\hline Rank & Institution & Salary & Rank & Institution & Salary \\
\hline $\begin{array}{r}1 \\
2 \\
3 \\
4 \\
4 \\
6 \\
7 \\
8 \\
9 \\
10\end{array}$ & $\begin{array}{l}\text { Princeton } \\
\text { Guelph } \\
\text { Kent State } \\
\text { Colorado State } \\
\text { New York University } \\
\text { Calgary } \\
\text { Harvard } \\
\text { Alberta } \\
\text { British Columbia } \\
\text { Saskatchewan }\end{array}$ & $\begin{array}{l}63,200 \\
58,392 \\
57,078 \\
55,000 \\
55,000 \\
54,945 \\
53,093 \\
52,833 \\
52,420 \\
52,202\end{array}$ & $\begin{array}{l}59 \\
59 \\
59 \\
59 \\
59 \\
59 \\
59 \\
59 \\
59 \\
59\end{array}$ & $\begin{array}{l}\text { Colorado } \\
\text { Georgia Tech } \\
\text { Houston } \\
\text { Iowa State } \\
\text { North Carolina } \\
\text { Northwestern } \\
\text { Notre Dame } \\
\text { Southern Illinois } \\
\text { Tennessee } \\
\text { Virginia }\end{array}$ & $\begin{array}{l}44,000 \\
44,000 \\
44,000 \\
44,000 \\
44,000 \\
44,000 \\
44,000 \\
44,000 \\
44,000 \\
44,000\end{array}$ \\
\hline $\begin{array}{l}11 \\
12 \\
12 \\
12 \\
15 \\
16 \\
17 \\
18 \\
19 \\
20\end{array}$ & $\begin{array}{l}\text { Brigham Young } \\
\text { Columbia } \\
\text { MIT } \\
\text { North Carolina State } \\
\text { Johns Hopkins } \\
\text { Arizona } \\
\text { Rutgers } \\
\text { Yale } \\
\text { Queen's } \\
\text { Chicago }\end{array}$ & $\begin{array}{l}52,020 \\
52,000 \\
52,000 \\
52,000 \\
51,027 \\
50,857 \\
50,765 \\
50,500 \\
50,446 \\
50,151\end{array}$ & $\begin{array}{l}69 \\
70 \\
71 \\
72 \\
73 \\
73 \\
73 \\
73 \\
77 \\
78\end{array}$ & $\begin{array}{l}\text { Pennsylvania State } \\
\text { Delaware } \\
\text { Montreal } \\
\text { Boston College } \\
\text { Arizona State } \\
\text { Kansas } \\
\text { Minnesota } \\
\text { SUNY Stony Brook } \\
\text { Washington } \\
\text { Massachusetts }\end{array}$ & $\begin{array}{l}43,709 \\
43,600 \\
43,497 \\
43,350 \\
43,000 \\
43,000 \\
43,000 \\
43,000 \\
42,600 \\
42,155\end{array}$ \\
\hline $\begin{array}{l}21 \\
21 \\
21 \\
24 \\
25 \\
26 \\
26 \\
28 \\
29 \\
30\end{array}$ & $\begin{array}{l}\text { Connecticut } \\
\text { Illinois, Urbana } \\
\text { Nebraska } \\
\text { Toronto } \\
\text { Waterloo } \\
\text { Southern California } \\
\text { Texas A\&M } \\
\text { Cornell } \\
\text { Western Ontario } \\
\text { Emory }\end{array}$ & $\begin{array}{l}50,000 \\
50,000 \\
50,000 \\
49,451 \\
49,110 \\
48,500 \\
48,500 \\
48,000 \\
47,836 \\
47,750\end{array}$ & $\begin{array}{l}79 \\
79 \\
79 \\
79 \\
79 \\
79 \\
85 \\
86 \\
86 \\
86\end{array}$ & $\begin{array}{l}\text { Alabama } \\
\text { Cincinnati } \\
\text { Florida } \\
\text { Florida State } \\
\text { Michigan } \\
\text { Oklahoma } \\
\text { Ohio University } \\
\text { Iowa } \\
\text { Kentucky } \\
\text { Pennsylvania }\end{array}$ & $\begin{array}{l}42,000 \\
42,000 \\
42,000 \\
42,000 \\
42,000 \\
42,000 \\
41,500 \\
41,000 \\
41,000 \\
41,000\end{array}$ \\
\hline $\begin{array}{l}31 \\
32 \\
33 \\
34 \\
35 \\
35 \\
35 \\
35 \\
39 \\
40\end{array}$ & $\begin{array}{l}\text { Ottawa } \\
\text { Michigan State } \\
\text { McGill } \\
\text { California, Riverside } \\
\text { George Washington } \\
\text { Illinois, Chicago } \\
\text { Purdue } \\
\text { SUNY Buffalo } \\
\text { Laval } \\
\text { York }\end{array}$ & $\begin{array}{l}47,538 \\
47,500 \\
47,366 \\
47,087 \\
47,000 \\
47,000 \\
47,000 \\
47,000 \\
46,937 \\
46,419\end{array}$ & $\begin{array}{l}86 \\
86 \\
91 \\
92 \\
93 \\
94 \\
94 \\
94 \\
94 \\
94\end{array}$ & $\begin{array}{l}\text { Vanderbilt } \\
\text { Wayne State } \\
\text { Wisconsin } \\
\text { Brown } \\
\text { Indiana } \\
\text { Maryland } \\
\text { Missouri } \\
\text { New Mexico } \\
\text { Oregon } \\
\text { Tulane }\end{array}$ & $\begin{array}{l}41,000 \\
41,000 \\
40,526 \\
40,500 \\
40,400 \\
40,000 \\
40,000 \\
40,000 \\
40,000 \\
40,000\end{array}$ \\
\hline $\begin{array}{l}41 \\
42 \\
42 \\
42 \\
42 \\
42 \\
47 \\
48 \\
49 \\
50\end{array}$ & $\begin{array}{l}\text { Manitoba } \\
\text { California, Berkeley } \\
\text { California, Davis } \\
\text { California, Los Angeles } \\
\text { California, San Diego } \\
\text { California, Santa Barbara } \\
\text { California, Irvine } \\
\text { Ohio State } \\
\text { McMaster } \\
\text { Dartmouth }\end{array}$ & $\begin{array}{l}46,249 \\
46,164 \\
46,164 \\
46,164 \\
46,164 \\
46,164 \\
46,144 \\
46,000 \\
45,945 \\
45,500\end{array}$ & $\begin{array}{r}94 \\
94 \\
101 \\
102 \\
103 \\
104 \\
105 \\
105 \\
105 \\
105\end{array}$ & $\begin{array}{l}\text { Virginia Tech } \\
\text { Washington U.-St. Louis } \\
\text { SUNY Albany } \\
\text { Rochester } \\
\text { Rice } \\
\text { Washington State } \\
\text { Georgia } \\
\text { Louisiana State } \\
\text { Oklahoma State } \\
\text { South Carolina }\end{array}$ & $\begin{array}{l}40,000 \\
40,000 \\
39,350 \\
38,983 \\
38,700 \\
38,500 \\
38,000 \\
38,000 \\
38,000 \\
38,000\end{array}$ \\
\hline $\begin{array}{l}51 \\
51 \\
51 \\
51 \\
51 \\
51 \\
57 \\
58\end{array}$ & $\begin{array}{l}\text { Duke } \\
\text { Georgetown } \\
\text { Miami } \\
\text { Texas } \\
\text { Texas Tech } \\
\text { Utah } \\
\text { Auburn } \\
\text { Temple }\end{array}$ & $\begin{array}{l}45,000 \\
45,000 \\
45,000 \\
45,000 \\
45,000 \\
45,000 \\
44,720 \\
44,004\end{array}$ & $\begin{array}{l}105 \\
110 \\
111 \\
111 \\
113 \\
114 \\
115\end{array}$ & $\begin{array}{l}\text { Syracuse } \\
\text { Louisville } \\
\text { Case Western Reserve } \\
\text { Hawaii } \\
\text { Howard } \\
\text { Pittsburgh } \\
\text { Boston University }\end{array}$ & $\begin{array}{l}38,000 \\
37,000 \\
35,000 \\
35,000 \\
34,627 \\
34,000 \\
33,000\end{array}$ \\
\hline
\end{tabular}

Beginning salary figures represent officially designated base, not necessarily salaries of actual incumbents.

Excludes medical and law libraries. See Tables 36 and 43 for comparable figures for medical and law libraries.

Canadian salaries are expressed in US dollars. 
Table 10: Median Professional Salaries in ARL University Libraries

Rank Order Table, FY 2009-2010

\begin{tabular}{|c|c|c|c|c|c|}
\hline Rank & Institution & Salary & Rank & Institution & Salary \\
\hline $\begin{array}{r}1 \\
2 \\
3 \\
4 \\
5 \\
6 \\
7 \\
8 \\
9 \\
10\end{array}$ & $\begin{array}{l}\text { Rutgers } \\
\text { Alberta } \\
\text { California, Davis } \\
\text { Manitoba } \\
\text { Queen's } \\
\text { California, Berkeley } \\
\text { Saskatchewan } \\
\text { Connecticut } \\
\text { York } \\
\text { Calgary }\end{array}$ & $\begin{array}{l}91,751 \\
89,319 \\
88,488 \\
85,771 \\
83,438 \\
82,524 \\
78,850 \\
78,079 \\
77,321 \\
76,749\end{array}$ & $\begin{array}{l}58 \\
59 \\
60 \\
61 \\
62 \\
63 \\
64 \\
65 \\
66 \\
67\end{array}$ & $\begin{array}{l}\text { Pennsylvania } \\
\text { Miami } \\
\text { Iowa State } \\
\text { Illinois, Urbana } \\
\text { Brown } \\
\text { Pittsburgh } \\
\text { Syracuse } \\
\text { Purdue } \\
\text { Kent State } \\
\text { Duke }\end{array}$ & $\begin{array}{l}61,850 \\
61,728 \\
61,664 \\
61,645 \\
61,549 \\
60,711 \\
60,648 \\
60,513 \\
60,301 \\
60,000\end{array}$ \\
\hline $\begin{array}{l}11 \\
12 \\
12 \\
12 \\
12 \\
16 \\
17 \\
18 \\
19 \\
20\end{array}$ & $\begin{array}{l}\text { Yale } \\
\text { California, Irvine } \\
\text { California, Los Angeles } \\
\text { California, Riverside } \\
\text { California, San Diego } \\
\text { SUNY Stony Brook } \\
\text { Toronto } \\
\text { Princeton } \\
\text { Delaware } \\
\text { Chicago }\end{array}$ & $\begin{array}{l}76,300 \\
75,708 \\
75,708 \\
75,708 \\
75,708 \\
75,457 \\
75,194 \\
73,799 \\
73,353 \\
72,329\end{array}$ & $\begin{array}{l}67 \\
69 \\
70 \\
71 \\
72 \\
73 \\
74 \\
75 \\
76 \\
77\end{array}$ & $\begin{array}{l}\text { Virginia } \\
\text { Washington } \\
\text { Arizona } \\
\text { Kentucky } \\
\text { Montreal } \\
\text { Indiana } \\
\text { Texas } \\
\text { SUNY Albany } \\
\text { Wisconsin } \\
\text { Virginia Tech }\end{array}$ & $\begin{array}{l}60,000 \\
59,934 \\
59,915 \\
59,839 \\
59,349 \\
59,331 \\
59,260 \\
58,822 \\
58,654 \\
58,540\end{array}$ \\
\hline $\begin{array}{l}21 \\
22 \\
23 \\
24 \\
25 \\
26 \\
27 \\
28 \\
29 \\
30\end{array}$ & $\begin{array}{l}\text { Massachusetts } \\
\text { MIT } \\
\text { Harvard } \\
\text { Waterloo } \\
\text { British Columbia } \\
\text { New York University } \\
\text { SUNY Buffalo } \\
\text { California, Santa Barbara } \\
\text { New Mexico } \\
\text { Dartmouth }\end{array}$ & $\begin{array}{l}72,263 \\
70,959 \\
70,720 \\
70,260 \\
70,005 \\
69,989 \\
69,048 \\
68,892 \\
68,853 \\
67,933\end{array}$ & $\begin{array}{l}78 \\
79 \\
80 \\
81 \\
82 \\
83 \\
84 \\
85 \\
86 \\
87\end{array}$ & $\begin{array}{l}\text { Washington State } \\
\text { Temple } \\
\text { Georgia Tech } \\
\text { McGill } \\
\text { Nebraska } \\
\text { Texas Tech } \\
\text { Boston University } \\
\text { Houston } \\
\text { Illinois, Chicago } \\
\text { Florida }\end{array}$ & $\begin{array}{l}58,172 \\
58,140 \\
58,016 \\
57,834 \\
57,472 \\
57,135 \\
56,600 \\
56,563 \\
56,555 \\
56,388\end{array}$ \\
\hline $\begin{array}{l}31 \\
32 \\
33 \\
34 \\
35 \\
36 \\
37 \\
38 \\
39 \\
40\end{array}$ & $\begin{array}{l}\text { Southern California } \\
\text { Boston College } \\
\text { George Washington } \\
\text { Guelph } \\
\text { Maryland } \\
\text { Notre Dame } \\
\text { Tennessee } \\
\text { Hawaii } \\
\text { Michigan State } \\
\text { Johns Hopkins }\end{array}$ & $\begin{array}{l}67,650 \\
67,280 \\
67,143 \\
66,902 \\
66,603 \\
66,508 \\
66,307 \\
66,225 \\
65,850 \\
65,690\end{array}$ & $\begin{array}{l}88 \\
89 \\
90 \\
91 \\
92 \\
93 \\
94 \\
95 \\
96 \\
97\end{array}$ & $\begin{array}{l}\text { Tulane } \\
\text { Utah } \\
\text { Oregon } \\
\text { Missouri } \\
\text { Louisville } \\
\text { Rice } \\
\text { Western Ontario } \\
\text { Texas A\&M } \\
\text { Iowa } \\
\text { Case Western Reserve }\end{array}$ & $\begin{array}{l}56,325 \\
56,312 \\
55,973 \\
55,964 \\
55,884 \\
55,833 \\
55,725 \\
55,704 \\
55,573 \\
55,227\end{array}$ \\
\hline $\begin{array}{l}41 \\
42 \\
43 \\
44 \\
45 \\
46 \\
47 \\
48 \\
49 \\
50\end{array}$ & $\begin{array}{l}\text { Colorado State } \\
\text { Arizona State } \\
\text { North Carolina State } \\
\text { Colorado } \\
\text { Columbia } \\
\text { Cornell } \\
\text { Pennsylvania State } \\
\text { Brigham Young } \\
\text { Michigan } \\
\text { North Carolina }\end{array}$ & $\begin{array}{l}65,300 \\
65,071 \\
65,000 \\
64,573 \\
64,385 \\
64,239 \\
64,24 \\
63,950 \\
63,717 \\
63,712\end{array}$ & $\begin{array}{r}98 \\
99 \\
100 \\
101 \\
102 \\
103 \\
104 \\
105 \\
106 \\
107\end{array}$ & $\begin{array}{l}\text { Kansas } \\
\text { Wayne State } \\
\text { Vanderbilt } \\
\text { Ohio State } \\
\text { Oklahoma } \\
\text { Washington U.-St. Louis } \\
\text { Auburn } \\
\text { Rochester } \\
\text { Oklahoma State } \\
\text { Southern Illinois }\end{array}$ & $\begin{array}{l}54,806 \\
54,705 \\
54,655 \\
54,450 \\
53,742 \\
53,740 \\
53,705 \\
53,000 \\
52,657 \\
52,536\end{array}$ \\
\hline $\begin{array}{l}51 \\
52 \\
53 \\
54 \\
55 \\
56 \\
57\end{array}$ & $\begin{array}{l}\text { Minnesota } \\
\text { Georgetown } \\
\text { Northwestern } \\
\text { Emory } \\
\text { McMaster } \\
\text { Laval } \\
\text { Cincinnati }\end{array}$ & $\begin{array}{l}63,557 \\
63,538 \\
63,429 \\
63,068 \\
62,508 \\
62,474 \\
61,879\end{array}$ & $\begin{array}{l}108 \\
109 \\
110 \\
111 \\
112 \\
113 \\
114\end{array}$ & $\begin{array}{l}\text { Alabama } \\
\text { Georgia } \\
\text { South Carolina } \\
\text { Florida State } \\
\text { Ohio University } \\
\text { Louisiana State } \\
\text { Howard }\end{array}$ & $\begin{array}{l}52,204 \\
50,000 \\
49,838 \\
49,805 \\
48,787 \\
46,462 \\
43,010\end{array}$ \\
\hline
\end{tabular}

Reprinted from ARL Annual Salary Survey 2009-2010. Salaries of directors are not included in the calculation of medians. Excludes medical and law libraries. See Tables 37 and 44 for comparable figures for medical and law libraries.

Canadian salaries are expressed in US dollars. 
Table 11: Median Professional Salaries in ARL University Libraries

Rank Order Table, FY 2010-2011

\begin{tabular}{|c|c|c|c|c|c|}
\hline Rank & Institution & Salary & Rank & Institution & Salary \\
\hline $\begin{array}{r}1 \\
2 \\
3 \\
4 \\
5 \\
6 \\
7 \\
8 \\
9 \\
10\end{array}$ & $\begin{array}{l}\text { Queen's } \\
\text { Alberta } \\
\text { York } \\
\text { Manitoba } \\
\text { Rutgers } \\
\text { Calgary } \\
\text { California, Davis } \\
\text { Ottawa } \\
\text { British Columbia } \\
\text { California, Berkeley }\end{array}$ & $\begin{array}{l}99,005 \\
96,759 \\
96,446 \\
95,343 \\
91,751 \\
88,732 \\
88,488 \\
85,122 \\
83,131 \\
82,524\end{array}$ & $\begin{array}{l}59 \\
60 \\
61 \\
62 \\
63 \\
64 \\
65 \\
66 \\
67 \\
68\end{array}$ & $\begin{array}{l}\text { Kent State } \\
\text { Georgetown } \\
\text { Emory } \\
\text { Pennsylvania } \\
\text { Iowa State } \\
\text { Northwestern } \\
\text { Brown } \\
\text { Pittsburgh } \\
\text { SUNY Albany } \\
\text { North Carolina }\end{array}$ & $\begin{array}{l}64,520 \\
63,965 \\
63,198 \\
63,148 \\
63,105 \\
62,995 \\
62,972 \\
62,897 \\
62,729 \\
62,300\end{array}$ \\
\hline $\begin{array}{l}11 \\
12 \\
13 \\
14 \\
15 \\
16 \\
17 \\
18 \\
19 \\
20\end{array}$ & $\begin{array}{l}\text { Toronto } \\
\text { Saskatchewan } \\
\text { SUNY Stony Brook } \\
\text { Connecticut } \\
\text { California, Riverside } \\
\text { Guelph } \\
\text { Yale } \\
\text { Princeton } \\
\text { Waterloo } \\
\text { California, Irvine }\end{array}$ & $\begin{array}{l}82,254 \\
81,582 \\
79,599 \\
79,309 \\
79,116 \\
76,872 \\
76,365 \\
76,100 \\
75,974 \\
75,708\end{array}$ & $\begin{array}{l}69 \\
70 \\
71 \\
72 \\
73 \\
74 \\
75 \\
76 \\
77 \\
78\end{array}$ & $\begin{array}{l}\text { McMaster } \\
\text { Syracuse } \\
\text { Colorado } \\
\text { Purdue } \\
\text { Rice } \\
\text { Temple } \\
\text { Duke } \\
\text { Kentucky } \\
\text { Tulane } \\
\text { Indiana }\end{array}$ & $\begin{array}{l}62,118 \\
61,390 \\
61,262 \\
61,006 \\
60,300 \\
60,048 \\
60,000 \\
59,984 \\
59,492 \\
59,331\end{array}$ \\
\hline $\begin{array}{l}20 \\
20 \\
23 \\
24 \\
25 \\
26 \\
27 \\
28 \\
29 \\
30\end{array}$ & $\begin{array}{l}\text { California, Los Angeles } \\
\text { California, San Diego } \\
\text { California, Santa Barbara } \\
\text { Massachusetts } \\
\text { MIT } \\
\text { Chicago } \\
\text { Delaware } \\
\text { George Washington } \\
\text { Harvard } \\
\text { New York University }\end{array}$ & $\begin{array}{l}75,708 \\
75,708 \\
74,104 \\
73,553 \\
73,326 \\
73,130 \\
72,868 \\
72,277 \\
72,134 \\
71,616\end{array}$ & $\begin{array}{l}79 \\
80 \\
81 \\
82 \\
83 \\
84 \\
85 \\
86 \\
87 \\
88\end{array}$ & $\begin{array}{l}\text { Texas } \\
\text { Arizona } \\
\text { Texas A\&M } \\
\text { Washington } \\
\text { Virginia Tech } \\
\text { Washington State } \\
\text { Florida } \\
\text { Georgia Tech } \\
\text { Iowa } \\
\text { Oregon }\end{array}$ & $\begin{array}{l}59,304 \\
59,118 \\
59,055 \\
58,752 \\
58,540 \\
58,172 \\
58,073 \\
58,016 \\
57,840 \\
57,839\end{array}$ \\
\hline $\begin{array}{l}31 \\
32 \\
33 \\
34 \\
35 \\
36 \\
37 \\
38 \\
39 \\
40\end{array}$ & $\begin{array}{l}\text { SUNY Buffalo } \\
\text { Laval } \\
\text { Southern California } \\
\text { Boston College } \\
\text { Maryland } \\
\text { Notre Dame } \\
\text { New Mexico } \\
\text { Michigan State } \\
\text { Tennessee } \\
\text { Dartmouth }\end{array}$ & $\begin{array}{l}70,554 \\
69,944 \\
69,761 \\
69,560 \\
69,035 \\
67,915 \\
67,913 \\
67,373 \\
67,268 \\
67,159\end{array}$ & $\begin{array}{l}89 \\
90 \\
91 \\
92 \\
93 \\
94 \\
95 \\
96 \\
97 \\
98\end{array}$ & $\begin{array}{l}\text { Illinois, Chicago } \\
\text { Wisconsin } \\
\text { Nebraska } \\
\text { Kansas } \\
\text { Boston University } \\
\text { Missouri } \\
\text { Southern Illinois } \\
\text { Rochester } \\
\text { Vanderbilt } \\
\text { Washington U.-St. Louis }\end{array}$ & $\begin{array}{l}57,730 \\
57,692 \\
57,453 \\
57,417 \\
57,200 \\
57,127 \\
56,912 \\
56,059 \\
55,945 \\
55,489\end{array}$ \\
\hline $\begin{array}{l}41 \\
42 \\
43 \\
44 \\
45 \\
46 \\
47 \\
48 \\
49 \\
50\end{array}$ & $\begin{array}{l}\text { Western Ontario } \\
\text { Cornell } \\
\text { Cincinnati } \\
\text { Pennsylvania State } \\
\text { Brigham Young } \\
\text { Michigan } \\
\text { Minnesota } \\
\text { Montreal } \\
\text { Johns Hopkins } \\
\text { Columbia }\end{array}$ & $\begin{array}{l}66,961 \\
66,873 \\
66,260 \\
66,258 \\
66,000 \\
65,884 \\
65,785 \\
65,596 \\
65,353 \\
65,280\end{array}$ & $\begin{array}{r}99 \\
100 \\
101 \\
102 \\
103 \\
104 \\
105 \\
106 \\
107 \\
108\end{array}$ & $\begin{array}{l}\text { Case Western Reserve } \\
\text { Wayne State } \\
\text { Utah } \\
\text { Alabama } \\
\text { Houston } \\
\text { Texas Tech } \\
\text { Oklahoma } \\
\text { Ohio State } \\
\text { Louisville } \\
\text { Auburn }\end{array}$ & $\begin{array}{l}55,434 \\
55,396 \\
55,375 \\
55,188 \\
54,628 \\
54,163 \\
53,477 \\
53,292 \\
53,031 \\
52,330\end{array}$ \\
\hline $\begin{array}{l}51 \\
52 \\
53 \\
53 \\
55 \\
56 \\
57 \\
58\end{array}$ & $\begin{array}{l}\text { Illinois, Urbana } \\
\text { Arizona State } \\
\text { Miami } \\
\text { North Carolina State } \\
\text { McGill } \\
\text { Colorado State } \\
\text { Hawaii } \\
\text { Virginia }\end{array}$ & $\begin{array}{l}65,129 \\
65,039 \\
65,000 \\
65,000 \\
64,865 \\
64,700 \\
64,692 \\
64,600\end{array}$ & $\begin{array}{l}109 \\
110 \\
111 \\
112 \\
113 \\
114 \\
115\end{array}$ & $\begin{array}{l}\text { Florida State } \\
\text { Oklahoma State } \\
\text { Howard } \\
\text { Georgia } \\
\text { Ohio University } \\
\text { South Carolina } \\
\text { Louisiana State }\end{array}$ & $\begin{array}{l}52,265 \\
51,865 \\
50,223 \\
49,795 \\
49,275 \\
48,627 \\
46,901\end{array}$ \\
\hline
\end{tabular}

Salaries of directors are not included in the calculation of medians.

Excludes medical and law libraries. See Tables 37 and 44 for comparable figures for medical and law libraries.

Canadian salaries are expressed in US dollars. 
Table 12: Average Professional Salaries in ARL University Libraries

Rank Order Table, FY 2009-2010

\begin{tabular}{|c|c|c|c|c|c|}
\hline Rank & Institution & Salary & Rank & Institution & Salary \\
\hline $\begin{array}{r}1 \\
2 \\
3 \\
4 \\
5 \\
6 \\
7 \\
8 \\
9 \\
10\end{array}$ & $\begin{array}{l}\text { Rutgers } \\
\text { Alberta } \\
\text { California, Berkeley } \\
\text { York } \\
\text { Connecticut } \\
\text { Manitoba } \\
\text { Calgary } \\
\text { California, Davis } \\
\text { Yale } \\
\text { Queen's }\end{array}$ & $\begin{array}{l}89,058 \\
84,172 \\
83,853 \\
82,297 \\
82,083 \\
81,855 \\
81,275 \\
81,135 \\
81,088 \\
80,504\end{array}$ & $\begin{array}{l}58 \\
59 \\
60 \\
61 \\
62 \\
63 \\
64 \\
65 \\
66 \\
67\end{array}$ & $\begin{array}{l}\text { Pittsburgh } \\
\text { Washington } \\
\text { Arizona State } \\
\text { Brigham Young } \\
\text { Pennsylvania } \\
\text { Arizona } \\
\text { Brown } \\
\text { Syracuse } \\
\text { Purdue } \\
\text { Cincinnati }\end{array}$ & $\begin{array}{l}66,551 \\
66,476 \\
66,091 \\
66,005 \\
65,997 \\
65,897 \\
65,880 \\
65,847 \\
65,573 \\
65,445\end{array}$ \\
\hline $\begin{array}{l}11 \\
12 \\
13 \\
14 \\
15 \\
16 \\
17 \\
18 \\
19 \\
20\end{array}$ & $\begin{array}{l}\text { SUNY Stony Brook } \\
\text { New York University } \\
\text { Princeton } \\
\text { Toronto } \\
\text { California, Los Angeles } \\
\text { California, Riverside } \\
\text { Harvard } \\
\text { California, San Diego } \\
\text { California, Irvine } \\
\text { Saskatchewan }\end{array}$ & $\begin{array}{l}79,837 \\
79,018 \\
79,015 \\
78,211 \\
78,189 \\
77,661 \\
77,319 \\
77,158 \\
76,645 \\
76,307\end{array}$ & $\begin{array}{l}68 \\
69 \\
70 \\
71 \\
72 \\
73 \\
74 \\
75 \\
76 \\
77\end{array}$ & $\begin{array}{l}\text { Nebraska } \\
\text { Duke } \\
\text { Kent State } \\
\text { Indiana } \\
\text { McGill } \\
\text { Georgia Tech } \\
\text { Iowa State } \\
\text { Virginia Tech } \\
\text { Washington State } \\
\text { Montreal }\end{array}$ & $\begin{array}{l}65,057 \\
64,870 \\
63,997 \\
63,870 \\
63,797 \\
63,526 \\
63,217 \\
63,148 \\
62,755 \\
62,740\end{array}$ \\
\hline $\begin{array}{l}21 \\
22 \\
23 \\
24 \\
25 \\
26 \\
27 \\
28 \\
29 \\
30\end{array}$ & $\begin{array}{l}\text { Chicago } \\
\text { Delaware } \\
\text { Southern California } \\
\text { MIT } \\
\text { George Washington } \\
\text { British Columbia } \\
\text { North Carolina State } \\
\text { Columbia } \\
\text { New Mexico } \\
\text { SUNY Buffalo }\end{array}$ & $\begin{array}{l}76,254 \\
75,703 \\
74,479 \\
74,430 \\
74,353 \\
73,023 \\
73,019 \\
72,939 \\
72,938 \\
72,916\end{array}$ & $\begin{array}{l}78 \\
79 \\
80 \\
81 \\
82 \\
83 \\
84 \\
85 \\
86 \\
87\end{array}$ & $\begin{array}{l}\text { Iowa } \\
\text { Texas A\&M } \\
\text { Rice } \\
\text { Illinois, Chicago } \\
\text { Laval } \\
\text { Wisconsin } \\
\text { Houston } \\
\text { McMaster } \\
\text { Utah } \\
\text { Wayne State }\end{array}$ & $\begin{array}{l}62,529 \\
62,441 \\
62,215 \\
62,208 \\
61,963 \\
61,856 \\
61,669 \\
61,608 \\
61,470 \\
61,261\end{array}$ \\
\hline $\begin{array}{l}31 \\
32 \\
33 \\
34 \\
35 \\
36 \\
37 \\
38 \\
39 \\
40\end{array}$ & $\begin{array}{l}\text { Dartmouth } \\
\text { Cornell } \\
\text { California, Santa Barbara } \\
\text { Waterloo } \\
\text { Massachusetts } \\
\text { Boston College } \\
\text { Notre Dame } \\
\text { Tennessee } \\
\text { Georgetown } \\
\text { Guelph }\end{array}$ & $\begin{array}{l}72,208 \\
71,548 \\
71,378 \\
70,230 \\
69,882 \\
69,739 \\
69,492 \\
69,380 \\
69,227 \\
69,113\end{array}$ & $\begin{array}{l}88 \\
89 \\
90 \\
91 \\
92 \\
93 \\
94 \\
95 \\
96 \\
97\end{array}$ & $\begin{array}{l}\text { Kentucky } \\
\text { SUNY Albany } \\
\text { Kansas } \\
\text { Texas Tech } \\
\text { Tulane } \\
\text { Florida } \\
\text { Missouri } \\
\text { Oregon } \\
\text { Louisville } \\
\text { Ohio State }\end{array}$ & $\begin{array}{l}60,966 \\
60,964 \\
60,877 \\
60,690 \\
60,534 \\
60,450 \\
59,914 \\
59,782 \\
59,483 \\
59,476\end{array}$ \\
\hline $\begin{array}{l}41 \\
42 \\
43 \\
44 \\
45 \\
46 \\
47 \\
48 \\
49 \\
50\end{array}$ & $\begin{array}{l}\text { Johns Hopkins } \\
\text { Michigan State } \\
\text { Maryland } \\
\text { Emory } \\
\text { Colorado State } \\
\text { Miami } \\
\text { Virginia } \\
\text { Pennsylvania State } \\
\text { Northwestern } \\
\text { Michigan }\end{array}$ & $\begin{array}{l}68,926 \\
68,894 \\
68,874 \\
68,747 \\
68,311 \\
68,278 \\
67,988 \\
67,897 \\
67,873 \\
67,767\end{array}$ & $\begin{array}{r}98 \\
99 \\
100 \\
101 \\
102 \\
103 \\
104 \\
105 \\
106 \\
107\end{array}$ & $\begin{array}{l}\text { Western Ontario } \\
\text { Washington U.-St. Louis } \\
\text { Vanderbilt } \\
\text { Case Western Reserve } \\
\text { Auburn } \\
\text { Alabama } \\
\text { Southern Illinois } \\
\text { Boston University } \\
\text { Oklahoma State } \\
\text { Georgia }\end{array}$ & $\begin{array}{l}59,073 \\
59,037 \\
58,991 \\
58,788 \\
58,382 \\
58,272 \\
58,264 \\
57,615 \\
56,580 \\
56,544\end{array}$ \\
\hline $\begin{array}{l}51 \\
52 \\
53 \\
54 \\
55 \\
56 \\
57\end{array}$ & $\begin{array}{l}\text { North Carolina } \\
\text { Colorado } \\
\text { Texas } \\
\text { Temple } \\
\text { Minnesota } \\
\text { Illinois, Urbana } \\
\text { Hawaii }\end{array}$ & $\begin{array}{l}67,637 \\
67,523 \\
66,882 \\
66,849 \\
66,794 \\
66,755 \\
66,721\end{array}$ & $\begin{array}{l}108 \\
109 \\
110 \\
111 \\
112 \\
113 \\
114\end{array}$ & $\begin{array}{l}\text { Oklahoma } \\
\text { Rochester } \\
\text { Ohio University } \\
\text { Florida State } \\
\text { South Carolina } \\
\text { Louisiana State } \\
\text { Howard }\end{array}$ & $\begin{array}{l}55,853 \\
55,837 \\
55,522 \\
54,858 \\
53,658 \\
50,821 \\
43,547\end{array}$ \\
\hline
\end{tabular}

Reprinted from ARL Annual Salary Survey 2009-2010. Salaries of directors are not included in the calculation of averages. Excludes medical and law libraries. See Tables 38 and 45 for comparable figures for medical and law libraries.

Canadian salaries are expressed in US dollars. 
Table 13: Average Professional Salaries in ARL University Libraries

Rank ORder Table, FY 2010-2011

\begin{tabular}{|c|c|c|c|c|c|}
\hline Rank & Institution & Salary & Rank & Institution & Salary \\
\hline $\begin{array}{r}1 \\
2 \\
3 \\
4 \\
5 \\
6 \\
7 \\
8 \\
9 \\
10\end{array}$ & $\begin{array}{l}\text { York } \\
\text { Alberta } \\
\text { Queen's } \\
\text { Calgary } \\
\text { Manitoba } \\
\text { Rutgers } \\
\text { SUNY Stony Brook } \\
\text { Ottawa } \\
\text { Toronto } \\
\text { California, Berkeley }\end{array}$ & $\begin{array}{l}99,770 \\
96,215 \\
96,157 \\
91,776 \\
91,679 \\
91,176 \\
87,447 \\
87,360 \\
85,574 \\
85,329\end{array}$ & $\begin{array}{l}59 \\
60 \\
61 \\
62 \\
63 \\
64 \\
65 \\
66 \\
67 \\
68\end{array}$ & $\begin{array}{l}\text { Northwestern } \\
\text { Syracuse } \\
\text { Colorado State } \\
\text { Laval } \\
\text { Brigham Young } \\
\text { Texas } \\
\text { Pennsylvania } \\
\text { Kent State } \\
\text { Brown } \\
\text { Purdue }\end{array}$ & $\begin{array}{l}68,146 \\
67,893 \\
67,844 \\
67,439 \\
67,295 \\
66,964 \\
66,632 \\
66,624 \\
66,368 \\
65,562\end{array}$ \\
\hline $\begin{array}{l}11 \\
12 \\
13 \\
14 \\
15 \\
16 \\
17 \\
18 \\
19 \\
20\end{array}$ & $\begin{array}{l}\text { Saskatchewan } \\
\text { British Columbia } \\
\text { California, Davis } \\
\text { Princeton } \\
\text { Connecticut } \\
\text { Yale } \\
\text { Guelph } \\
\text { California, Riverside } \\
\text { California, Los Angeles } \\
\text { Harvard }\end{array}$ & $\begin{array}{l}85,160 \\
85,029 \\
84,334 \\
82,454 \\
81,341 \\
80,642 \\
80,588 \\
79,762 \\
79,113 \\
79,111\end{array}$ & $\begin{array}{l}69 \\
70 \\
71 \\
72 \\
73 \\
74 \\
75 \\
76 \\
77 \\
78\end{array}$ & $\begin{array}{l}\text { Duke } \\
\text { Washington } \\
\text { Iowa State } \\
\text { Arizona } \\
\text { Rice } \\
\text { North Carolina } \\
\text { Texas A\&M } \\
\text { Nebraska } \\
\text { Arizona State } \\
\text { Colorado }\end{array}$ & $\begin{array}{l}65,526 \\
65,466 \\
65,451 \\
65,351 \\
65,202 \\
65,091 \\
64,966 \\
64,928 \\
64,744 \\
64,595\end{array}$ \\
\hline $\begin{array}{l}21 \\
22 \\
23 \\
24 \\
25 \\
26 \\
27 \\
28 \\
29 \\
30\end{array}$ & $\begin{array}{l}\text { New York University } \\
\text { California, Irvine } \\
\text { MIT } \\
\text { California, San Diego } \\
\text { George Washington } \\
\text { Chicago } \\
\text { Delaware } \\
\text { Waterloo } \\
\text { Southern California } \\
\text { SUNY Buffalo }\end{array}$ & $\begin{array}{l}78,859 \\
77,825 \\
77,065 \\
77,064 \\
76,999 \\
76,692 \\
76,673 \\
76,645 \\
76,057 \\
75,265\end{array}$ & $\begin{array}{l}79 \\
80 \\
81 \\
82 \\
83 \\
84 \\
85 \\
86 \\
87 \\
88\end{array}$ & $\begin{array}{l}\text { Iowa } \\
\text { SUNY Albany } \\
\text { Indiana } \\
\text { Georgia Tech } \\
\text { Washington State } \\
\text { Illinois, Chicago } \\
\text { Virginia Tech } \\
\text { Hawaii } \\
\text { Tulane } \\
\text { Kansas }\end{array}$ & $\begin{array}{l}64,501 \\
64,024 \\
63,918 \\
63,534 \\
63,512 \\
63,420 \\
63,308 \\
63,037 \\
62,835 \\
62,232\end{array}$ \\
\hline $\begin{array}{l}31 \\
32 \\
33 \\
34 \\
35 \\
36 \\
37 \\
38 \\
39 \\
40\end{array}$ & $\begin{array}{l}\text { California, Santa Barbara } \\
\text { Cornell } \\
\text { Columbia } \\
\text { Massachusetts } \\
\text { North Carolina State } \\
\text { Notre Dame } \\
\text { McGill } \\
\text { Michigan } \\
\text { New Mexico } \\
\text { Michigan State }\end{array}$ & $\begin{array}{l}74,431 \\
74,377 \\
74,360 \\
72,957 \\
72,411 \\
71,751 \\
71,450 \\
71,354 \\
71,159 \\
70,979\end{array}$ & $\begin{array}{l}89 \\
90 \\
91 \\
92 \\
93 \\
94 \\
95 \\
96 \\
97 \\
98\end{array}$ & $\begin{array}{l}\text { Florida } \\
\text { Houston } \\
\text { Oregon } \\
\text { Wisconsin } \\
\text { Utah } \\
\text { Washington U.-St. Louis } \\
\text { Kentucky } \\
\text { Wayne State } \\
\text { Vanderbilt } \\
\text { Rochester }\end{array}$ & $\begin{array}{l}62,229 \\
62,188 \\
61,130 \\
61,068 \\
61,061 \\
60,832 \\
60,762 \\
60,697 \\
59,927 \\
59,814\end{array}$ \\
\hline $\begin{array}{l}41 \\
42 \\
43 \\
44 \\
45 \\
46 \\
47 \\
48 \\
49 \\
50\end{array}$ & $\begin{array}{l}\text { Boston College } \\
\text { Dartmouth } \\
\text { Montreal } \\
\text { Virginia } \\
\text { Georgetown } \\
\text { Maryland } \\
\text { Illinois, Urbana } \\
\text { Emory } \\
\text { Miami } \\
\text { Pennsylvania State }\end{array}$ & $\begin{array}{l}70,871 \\
70,594 \\
70,501 \\
70,351 \\
70,338 \\
69,981 \\
69,964 \\
69,761 \\
69,503 \\
69,465\end{array}$ & $\begin{array}{r}99 \\
100 \\
101 \\
102 \\
103 \\
104 \\
105 \\
106 \\
107 \\
108\end{array}$ & $\begin{array}{l}\text { Missouri } \\
\text { Boston University } \\
\text { Louisville } \\
\text { Southern Illinois } \\
\text { Case Western Reserve } \\
\text { Alabama } \\
\text { Texas Tech } \\
\text { Ohio State } \\
\text { Auburn } \\
\text { Oklahoma State }\end{array}$ & $\begin{array}{l}59,710 \\
59,680 \\
59,638 \\
59,157 \\
58,886 \\
58,760 \\
58,352 \\
57,846 \\
57,728 \\
56,678\end{array}$ \\
\hline $\begin{array}{l}51 \\
52 \\
53 \\
54 \\
55 \\
56 \\
57 \\
58\end{array}$ & $\begin{array}{l}\text { Johns Hopkins } \\
\text { Pittsburgh } \\
\text { Temple } \\
\text { McMaster } \\
\text { Tennessee } \\
\text { Western Ontario } \\
\text { Cincinnati } \\
\text { Minnesota }\end{array}$ & $\begin{array}{l}69,299 \\
69,211 \\
69,082 \\
69,041 \\
68,677 \\
68,581 \\
68,451 \\
68,210\end{array}$ & $\begin{array}{l}109 \\
110 \\
111 \\
112 \\
113 \\
114 \\
115\end{array}$ & $\begin{array}{l}\text { Howard } \\
\text { Florida State } \\
\text { Georgia } \\
\text { Ohio University } \\
\text { Oklahoma } \\
\text { South Carolina } \\
\text { Louisiana State }\end{array}$ & $\begin{array}{l}56,590 \\
56,313 \\
56,057 \\
55,590 \\
55,299 \\
52,032 \\
51,100\end{array}$ \\
\hline
\end{tabular}

Salaries of directors are not included in the calculation of averages.

Excludes medical and law libraries. See Tables 38 and 45 for comparable figures for medical and law libraries.

Canadian salaries are expressed in US dollars. 
Table 14: Average, Median, and Beginning Professional Salaries in ARL University Libraries

SuMMARY OF RANKINGS, FYs 2007-2008 TO 2010-2011

\begin{tabular}{|c|c|c|c|c|c|c|c|c|c|c|c|c|}
\hline \multirow{2}{*}{$\begin{array}{l}\text { Institution } \\
\text { FY }\end{array}$} & \multicolumn{4}{|c|}{ Average Salaries } & \multicolumn{4}{|c|}{ Median Salaries } & \multicolumn{4}{|c|}{ Beginning Salaries } \\
\hline & 2008 & 2009 & 2010 & 2011 & 2008 & 2009 & 2010 & 2011 & 2008 & 2009 & 2010 & 2011 \\
\hline Alabama & 92 & 97 & 103 & 104 & 96 & 105 & 108 & 102 & 74 & 73 & 72 & 79 \\
\hline Alberta & 11 & 4 & 2 & 2 & 2 & 1 & 2 & 2 & 38 & 8 & 60 & 8 \\
\hline Arizona & 56 & 65 & 63 & 72 & 56 & 62 & 70 & 80 & 7 & 26 & 16 & 16 \\
\hline Arizona State & 59 & 77 & 60 & 77 & 41 & 66 & 42 & 52 & 34 & 60 & 61 & 73 \\
\hline Auburn & 75 & 90 & 102 & 107 & 63 & 90 & 104 & 108 & 25 & 50 & 45 & 57 \\
\hline Boston University & 106 & 40 & 105 & 100 & 100 & 91 & 84 & 93 & 62 & 70 & 57 & 115 \\
\hline Boston College & 35 & 95 & 36 & 41 & 31 & 35 & 32 & 34 & 57 & 70 & 70 & 72 \\
\hline Brigham Young & 70 & 72 & 61 & 63 & 55 & 60 & 48 & 45 & 14 & 22 & 7 & 11 \\
\hline British Columbia & 26 & 10 & 26 & 12 & 22 & 9 & 25 & 9 & 15 & 2 & 21 & 9 \\
\hline Brown & 47 & 53 & 64 & 67 & 42 & 51 & 62 & 65 & 94 & 99 & 100 & 92 \\
\hline Calgary & $\mathrm{N} / \mathrm{A}$ & $\mathrm{N} / \mathrm{A}$ & 7 & 4 & $\mathrm{~N} / \mathrm{A}$ & $\mathrm{N} / \mathrm{A}$ & 10 & 6 & $\mathrm{~N} / \mathrm{A}$ & $\mathrm{N} / \mathrm{A}$ & 13 & 6 \\
\hline California, Berkeley & 3 & 8 & 3 & 10 & 5 & 10 & 6 & 10 & 69 & 35 & 22 & 42 \\
\hline California, Davis & 15 & 12 & 8 & 13 & 3 & 8 & 3 & 7 & 69 & 35 & 29 & 42 \\
\hline California, Irvine & 20 & 20 & 19 & 22 & 13 & 16 & 12 & 20 & 69 & 35 & 29 & 47 \\
\hline California, Los Angeles & 10 & 23 & 15 & 19 & 12 & 19 & 12 & 20 & 69 & 35 & 29 & 42 \\
\hline California, Riverside & 28 & 26 & 16 & 18 & 24 & 18 & 12 & 15 & 41 & 27 & 22 & 34 \\
\hline California, San Diego & 14 & 21 & 18 & 24 & 13 & 16 & 12 & 20 & 69 & 35 & 29 & 42 \\
\hline California, Santa Barbara & 23 & 32 & 33 & 31 & 27 & 31 & 28 & 23 & 43 & 35 & 29 & 42 \\
\hline Case Western Reserve & 96 & 98 & 101 & 103 & 90 & 96 & 97 & 99 & 108 & 110 & 111 & 111 \\
\hline Chicago & 18 & 25 & 21 & 26 & 23 & 28 & 20 & 26 & 12 & 21 & 18 & 20 \\
\hline Cincinnati & 49 & 60 & 67 & 57 & 47 & 64 & 57 & 43 & 94 & 100 & 92 & 79 \\
\hline Colorado & 46 & 61 & 52 & 78 & 49 & 48 & 44 & 71 & 58 & 51 & 50 & 59 \\
\hline Colorado State & 68 & 48 & 45 & 61 & 53 & 45 & 41 & 56 & 29 & 42 & 39 & 4 \\
\hline Columbia & 21 & 31 & 28 & 33 & 46 & 49 & 45 & 50 & 1 & 4 & 5 & 12 \\
\hline Connecticut & 7 & 11 & 5 & 15 & 9 & 12 & 8 & 14 & 5 & 22 & 19 & 21 \\
\hline Cornell & 33 & 41 & 32 & 32 & 40 & 55 & 46 & 42 & 16 & 28 & 24 & 28 \\
\hline Dartmouth & 19 & 30 & 31 & 42 & 16 & 30 & 30 & 40 & 74 & 67 & 37 & 50 \\
\hline Delaware & 29 & 29 & 22 & 27 & 34 & 24 & 19 & 27 & 28 & 58 & 58 & 70 \\
\hline Duke & 76 & 78 & 69 & 69 & 81 & 78 & 67 & 75 & 34 & 51 & 39 & 51 \\
\hline Emory & 57 & 46 & 44 & 48 & 51 & 54 & 54 & 61 & 94 & 83 & 72 & 30 \\
\hline Florida & 100 & 96 & 93 & 89 & 98 & 92 & 87 & 85 & 44 & 73 & 72 & 79 \\
\hline Florida State & 113 & 113 & 111 & 110 & 111 & 113 & 111 & 109 & 44 & 73 & 72 & 79 \\
\hline George Washington & 54 & 33 & 25 & 25 & 62 & 44 & 33 & 28 & 44 & 28 & 24 & 35 \\
\hline Georgetown & 40 & 39 & 39 & 45 & 48 & 56 & 52 & 60 & 16 & 43 & 39 & 51 \\
\hline Georgia & 97 & 99 & 107 & 111 & 106 & 106 & 109 & 112 & 108 & 105 & 104 & 105 \\
\hline Georgia Tech & 90 & 86 & 73 & 82 & 86 & 86 & 80 & 86 & 44 & 73 & 50 & 59 \\
\hline Guelph & 69 & 19 & 40 & 17 & 66 & 23 & 34 & 16 & 90 & 1 & 6 & 2 \\
\hline Harvard & 16 & 16 & 17 & 20 & 26 & 25 & 23 & 29 & 8 & 18 & 15 & 7 \\
\hline Hawaii & 74 & 54 & 57 & 86 & 54 & 33 & 38 & 57 & 42 & 65 & 66 & 111 \\
\hline
\end{tabular}

38 · ARL Annual Salary Survey 2010-2011 
Table 14: Average, Median, and Beginning Professional Salaries in ARL University Libraries

SuMMARY OF RANKINGS, FYs 2007-2008 TO 2010-2011

\begin{tabular}{|c|c|c|c|c|c|c|c|c|c|c|c|c|}
\hline \multirow{2}{*}{$\begin{array}{l}\text { Institution } \\
\text { FY }\end{array}$} & \multicolumn{4}{|c|}{ Average Salaries } & \multicolumn{4}{|c|}{ Median Salaries } & \multicolumn{4}{|c|}{ Beginning Salaries } \\
\hline & 2008 & 2009 & 2010 & 2011 & 2008 & 2009 & 2010 & 2011 & 2008 & 2009 & 2010 & 2011 \\
\hline Houston & 99 & 75 & 84 & 90 & 108 & 59 & 85 & 103 & 74 & 73 & 61 & 59 \\
\hline Howard & 108 & 110 & 114 & 109 & 93 & 101 & 114 & 111 & 107 & 108 & 104 & 113 \\
\hline Illinois, Chicago & 89 & 83 & 81 & 84 & 84 & 85 & 86 & 89 & 9 & 28 & 24 & 35 \\
\hline Illinois, Urbana & 62 & 57 & 56 & 47 & 68 & 61 & 61 & 51 & 26 & 41 & 34 & 21 \\
\hline Indiana & 60 & 74 & 71 & 81 & 60 & 70 & 73 & 78 & 74 & 90 & 90 & 93 \\
\hline Iowa & 65 & 76 & 78 & 79 & 79 & 83 & 96 & 87 & 74 & 85 & 83 & 86 \\
\hline Iowa State & 82 & 80 & 74 & 71 & 75 & 74 & 60 & 63 & 39 & 60 & 61 & 59 \\
\hline Johns Hopkins & 44 & 55 & 41 & 51 & 57 & 53 & 40 & 49 & 4 & 9 & 10 & 15 \\
\hline Kansas & 86 & 85 & 90 & 88 & 82 & 87 & 98 & 92 & 74 & 16 & 7 & 73 \\
\hline Kent State & 101 & 101 & 70 & 66 & 92 & 99 & 66 & 59 & 31 & 59 & 2 & 3 \\
\hline Kentucky & 85 & 87 & 88 & 95 & 71 & 76 & 71 & 76 & 91 & 85 & 83 & 86 \\
\hline Laval & 84 & 47 & 82 & 62 & 39 & 29 & 56 & 32 & 68 & 24 & 68 & 39 \\
\hline Louisiana State & 112 & 112 & 113 & 115 & 113 & 112 & 113 & 115 & 94 & 105 & 104 & 105 \\
\hline Louisville & 51 & 81 & 96 & 101 & 43 & 82 & 92 & 107 & 100 & 107 & 109 & 110 \\
\hline McGill & 43 & 28 & 72 & 37 & 32 & 34 & 81 & 55 & 65 & 13 & 65 & 33 \\
\hline McMaster & 37 & 14 & 85 & 54 & 20 & 11 & 55 & 69 & 89 & 49 & 91 & 49 \\
\hline Manitoba & 5 & 3 & 6 & 5 & 4 & 2 & 4 & 4 & 55 & 33 & 81 & 41 \\
\hline Maryland & 55 & 52 & 43 & 46 & 44 & 39 & 35 & 35 & 44 & 73 & 92 & 94 \\
\hline Massachusetts & 27 & 35 & 35 & 34 & 19 & 21 & 21 & 24 & 63 & 72 & 71 & 78 \\
\hline MIT & 25 & 24 & 24 & 23 & 29 & 25 & 22 & 25 & 5 & 6 & 4 & 12 \\
\hline Miami & 52 & 45 & 46 & 49 & 58 & 46 & 59 & 53 & 16 & 43 & 39 & 51 \\
\hline Michigan & 41 & 37 & 50 & 38 & 36 & 41 & 49 & 46 & 58 & 73 & 72 & 79 \\
\hline Michigan State & 71 & 73 & 42 & 40 & 76 & 71 & 39 & 38 & 9 & 28 & 24 & 32 \\
\hline Minnesota & 42 & 51 & 55 & 58 & 35 & 43 & 51 & 47 & 74 & 91 & 72 & 73 \\
\hline Missouri & 95 & 106 & 94 & 99 & 104 & 110 & 91 & 94 & 91 & 91 & 92 & 94 \\
\hline Montreal & 78 & 43 & 77 & 43 & 69 & 38 & 72 & 48 & 104 & 82 & 110 & 71 \\
\hline Nebraska & 73 & 69 & 68 & 76 & 87 & 80 & 82 & 91 & 16 & 10 & 11 & 21 \\
\hline New Mexico & 9 & 15 & 29 & 39 & 8 & 15 & 29 & 37 & 74 & 91 & 92 & 94 \\
\hline New York & 4 & 13 & 12 & 21 & 21 & 27 & 26 & 30 & 2 & 3 & 3 & 4 \\
\hline North Carolina & 34 & 58 & 51 & 74 & 37 & 52 & 50 & 68 & 44 & 51 & 50 & 59 \\
\hline North Carolina State & 30 & 34 & 27 & 35 & 45 & 47 & 43 & 53 & 9 & 16 & 11 & 12 \\
\hline Northwestern & 50 & 66 & 49 & 59 & 50 & 57 & 53 & 64 & 86 & 73 & 61 & 59 \\
\hline Notre Dame & 38 & 49 & 37 & 36 & 28 & 40 & 36 & 36 & 74 & 91 & 82 & 59 \\
\hline Ohio University & 111 & 100 & 110 & 112 & 112 & 111 & 112 & 113 & 105 & 83 & 83 & 85 \\
\hline Ohio State & 98 & 109 & 97 & 106 & 95 & 103 & 101 & 106 & 54 & 60 & 34 & 48 \\
\hline Oklahoma & 107 & 108 & 108 & 113 & 103 & 104 & 102 & 105 & 74 & 73 & 72 & 79 \\
\hline Oklahoma State & 104 & 104 & 106 & 108 & 105 & 100 & 106 & 110 & 105 & 108 & 104 & 105 \\
\hline Oregon & 103 & 103 & 95 & 91 & 102 & 98 & 90 & 88 & 108 & 91 & 92 & 94 \\
\hline Ottawa* & $\mathrm{N} / \mathrm{A}$ & $\mathrm{N} / \mathrm{A}$ & $\mathrm{N} / \mathrm{A}$ & 8 & $\mathrm{~N} / \mathrm{A}$ & $\mathrm{N} / \mathrm{A}$ & $\mathrm{N} / \mathrm{A}$ & 8 & $\mathrm{~N} / \mathrm{A}$ & $\mathrm{N} / \mathrm{A}$ & $\mathrm{N} / \mathrm{A}$ & 31 \\
\hline
\end{tabular}


Table 14: Average, Median, and Beginning Professional Salaries in ARL University Libraries

SuMMARY OF RANKINGS, FYs 2007-2008 TO 2010-2011

\begin{tabular}{|c|c|c|c|c|c|c|c|c|c|c|c|c|}
\hline \multirow{2}{*}{$\begin{array}{ll}\text { Institution } & \\
& \text { FY }\end{array}$} & \multicolumn{4}{|c|}{ Average Salaries } & \multicolumn{4}{|c|}{ Median Salaries } & \multicolumn{4}{|c|}{ Beginning Salaries } \\
\hline & 2008 & 2009 & 2010 & 2011 & 2008 & 2009 & 2010 & 2011 & 2008 & 2009 & 2010 & 2011 \\
\hline Pennsylvania & 66 & 68 & 62 & 65 & 64 & 65 & 58 & 62 & 39 & 67 & 59 & 86 \\
\hline Pennsylvania State & 39 & 42 & 48 & 50 & 38 & 42 & 47 & 44 & 56 & 69 & 69 & 69 \\
\hline Pittsburgh & 61 & 63 & 58 & 52 & 65 & 73 & 63 & 66 & 111 & 111 & 112 & 114 \\
\hline Princeton & 13 & 17 & 13 & 14 & 18 & 22 & 18 & 18 & 16 & 10 & 1 & 1 \\
\hline Purdue & 48 & 59 & 66 & 68 & 72 & 66 & 65 & 72 & 29 & 43 & 24 & 35 \\
\hline Queen's & 17 & 6 & 10 & 3 & 11 & 5 & 5 & 1 & 64 & 13 & 48 & 19 \\
\hline Rice & 72 & 82 & 80 & 73 & 83 & 89 & 93 & 73 & 85 & 103 & 108 & 103 \\
\hline Rochester & 102 & 105 & 109 & 98 & 101 & 102 & 105 & 96 & 103 & 104 & 103 & 102 \\
\hline Rutgers & 1 & 5 & 1 & 6 & 1 & 3 & 1 & 5 & 13 & 25 & 14 & 17 \\
\hline Saskatchewan & 12 & 7 & 20 & 11 & 10 & 7 & 7 & 12 & 53 & 12 & 38 & 10 \\
\hline South Carolina & 110 & 111 & 112 & 114 & 109 & 108 & 110 & 114 & 111 & 111 & 112 & 105 \\
\hline Southern California & 22 & 27 & 23 & 29 & 33 & 36 & 31 & 33 & 16 & 20 & 17 & 26 \\
\hline Southern Illinois & 105 & 102 & 104 & 102 & 107 & 109 & 107 & 95 & 58 & 60 & 50 & 59 \\
\hline SUNY Albany & 77 & 56 & 89 & 80 & 77 & 75 & 75 & 67 & 94 & 101 & 101 & 101 \\
\hline SUNY Buffalo & 58 & 38 & 30 & 30 & 52 & 37 & 27 & 31 & 16 & 43 & 39 & 35 \\
\hline SUNY Stony Brook & 24 & 22 & 11 & 7 & 25 & 20 & 16 & 13 & 34 & 51 & 72 & 73 \\
\hline Syracuse & 53 & 71 & 65 & 60 & 67 & 63 & 64 & 70 & $\mathrm{~N} / \mathrm{A}$ & 113 & $\mathrm{~N} / \mathrm{A}$ & 105 \\
\hline Temple & 45 & 67 & 54 & 53 & 78 & 88 & 79 & 74 & 86 & 64 & 49 & 58 \\
\hline Tennessee & 32 & 36 & 38 & 55 & 30 & 32 & 37 & 39 & 44 & 51 & 50 & 59 \\
\hline Texas & 64 & 62 & 53 & 64 & 73 & 77 & 74 & 79 & 16 & 43 & 34 & 51 \\
\hline Texas A\&M & 79 & 94 & 79 & 75 & 85 & 97 & 95 & 81 & 27 & 28 & 20 & 26 \\
\hline Texas Tech & 81 & 88 & 91 & 105 & 94 & 93 & 83 & 104 & 16 & 43 & 39 & 51 \\
\hline Toronto & 8 & 2 & 14 & 9 & 6 & 4 & 17 & 11 & 33 & 5 & 46 & 24 \\
\hline Tulane & 87 & 79 & 92 & 87 & 80 & 71 & 88 & 77 & 100 & 91 & 92 & 94 \\
\hline Utah & 80 & 84 & 86 & 93 & 88 & 81 & 89 & 101 & 44 & 51 & 50 & 51 \\
\hline Vanderbilt & 94 & 92 & 100 & 97 & 97 & 94 & 100 & 97 & 86 & 91 & 88 & 86 \\
\hline Virginia & 36 & 44 & 47 & 44 & 58 & 68 & 67 & 58 & 34 & 51 & 50 & 59 \\
\hline Virginia Tech & 67 & 70 & 75 & 85 & 61 & 58 & 77 & 83 & 91 & 85 & 92 & 94 \\
\hline Washington & 63 & 64 & 59 & 70 & 70 & 69 & 69 & 82 & 58 & 66 & 67 & 77 \\
\hline Washington State & 91 & 91 & 76 & 83 & 89 & 84 & 78 & 84 & 94 & 101 & 102 & 104 \\
\hline Washington U.-St. Louis & 93 & 93 & 99 & 94 & 99 & 95 & 103 & 98 & 100 & 91 & 92 & 94 \\
\hline Waterloo & 31 & 18 & 34 & 28 & 17 & 13 & 24 & 19 & 52 & 15 & 47 & 25 \\
\hline Wayne State & 109 & 107 & 87 & 96 & 110 & 107 & 99 & 100 & 67 & 89 & 88 & 86 \\
\hline Western Ontario & 88 & 50 & 98 & 56 & 91 & 50 & 94 & 41 & 65 & 34 & 86 & 29 \\
\hline Wisconsin & 83 & 89 & 83 & 92 & 74 & 79 & 76 & 90 & 84 & 88 & 87 & 91 \\
\hline Yale & 6 & 9 & 9 & 16 & 15 & 14 & 11 & 17 & 3 & 7 & 9 & 18 \\
\hline York & 2 & 1 & 4 & 1 & 7 & 6 & 9 & 3 & 32 & 19 & 80 & 40 \\
\hline
\end{tabular}

Excludes medical and law libraries.

${ }^{*}$ Ottawa became a member in 2010. 
Page Intentionally Left Blank.

ARL University Libraries · 41 
Table 15: Distribution of Professional Staff in ARL University Libraries

by SALARY AND Position, FY 2010-2011

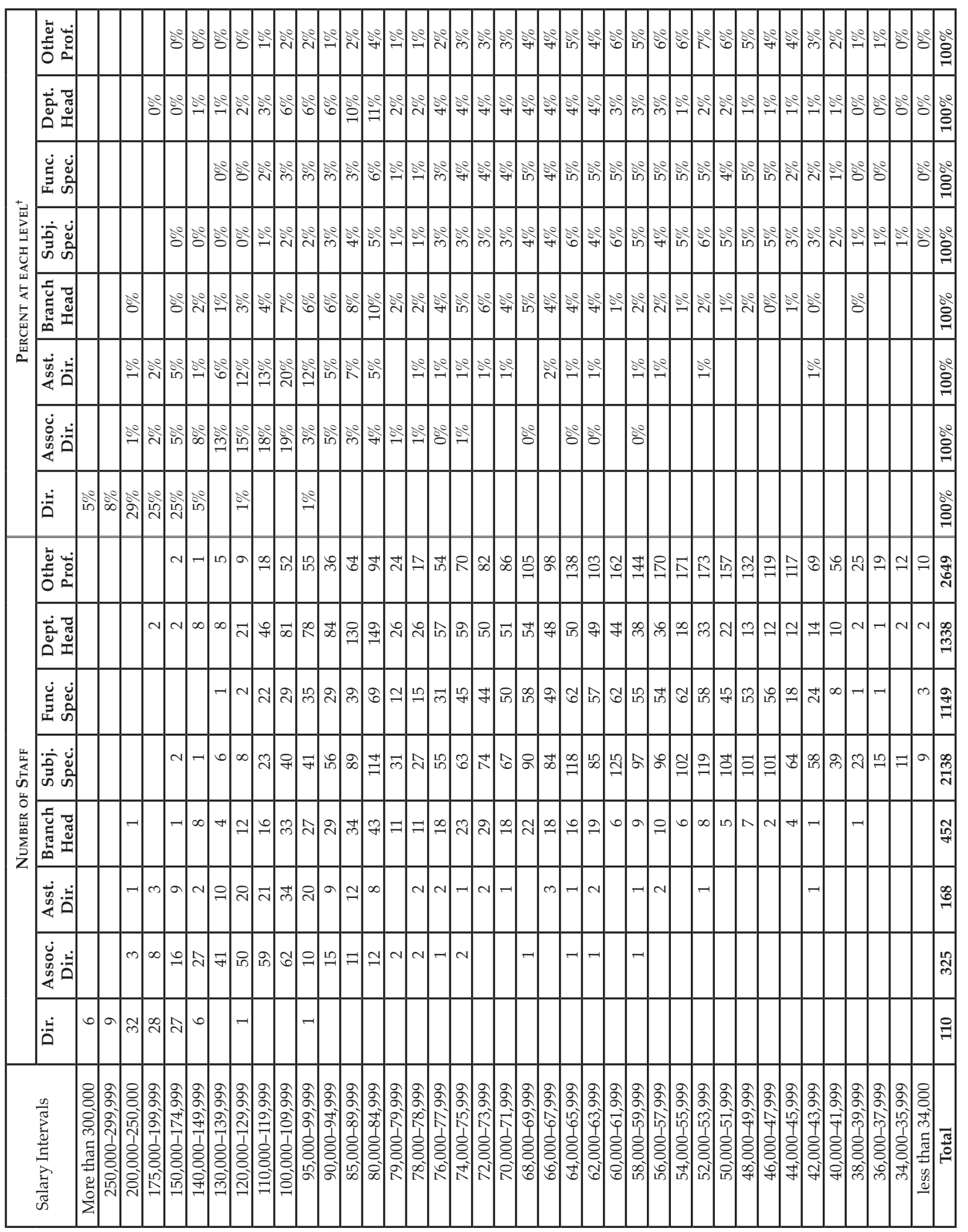

Excludes medical and law libraries.

+ A " 0 " percentage indicates less than one-half of one percent. 
Table 16: Distribution of Professional Staff in ARL University Libraries by Salary, Sex, and Position, FY 2010-2011

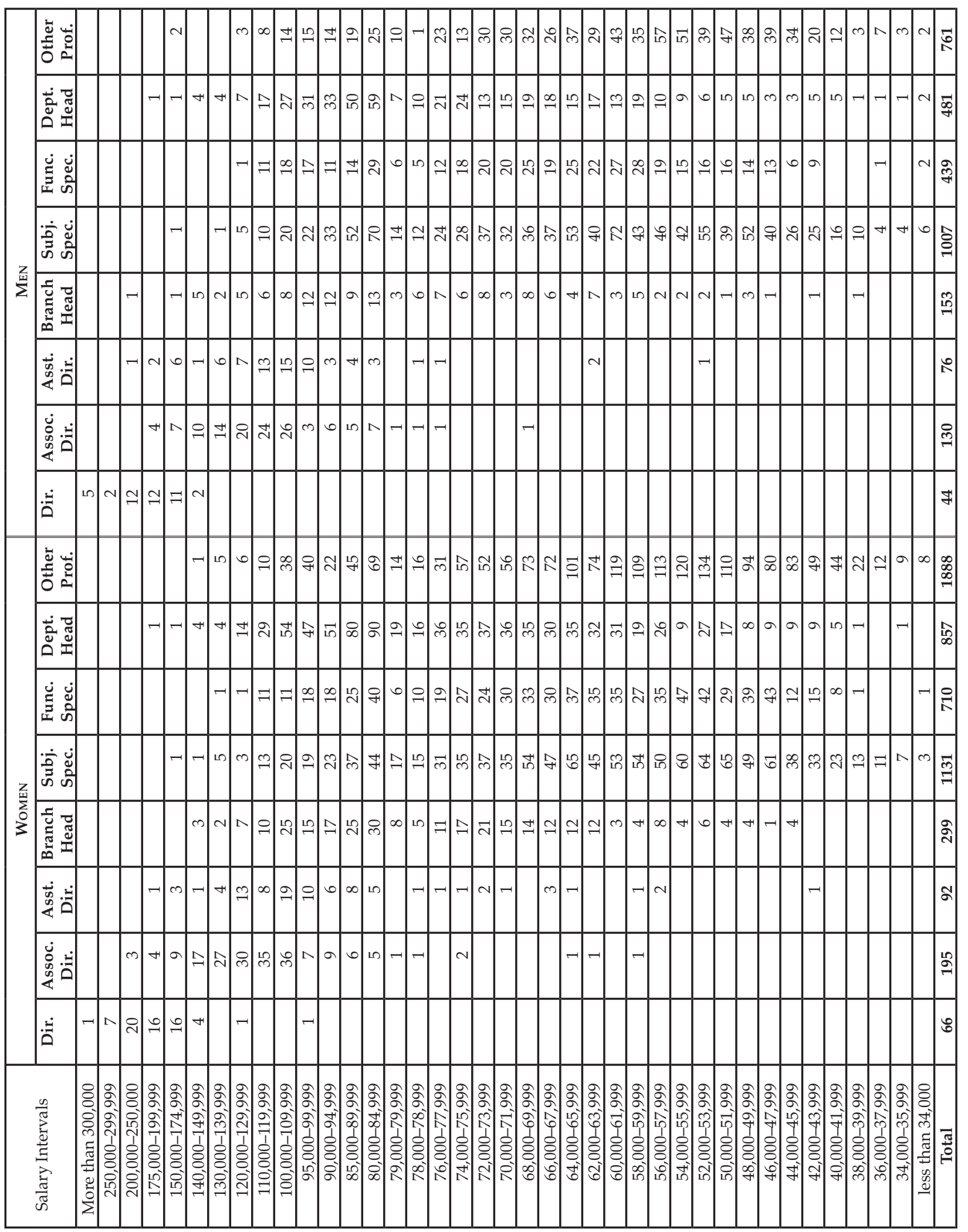

Excludes medical and law libraries. 
Table 17: Number and Average Salaries of ARL University Librarians by Position and Sex, FY 2010-2011

\begin{tabular}{|c|c|c|c|c|c|c|}
\hline \multirow[b]{2}{*}{ Position } & \multicolumn{2}{|c|}{ WOMEN } & \multicolumn{2}{|c|}{ Men } & \multicolumn{2}{|c|}{ TOTAL } \\
\hline & Salary & No. & Salary & No. & Salary & No. \\
\hline Director & $\$ 196,635$ & 66 & $\$ 207,959$ & 44 & $\$ 201,165$ & 110 \\
\hline Associate Director & 120,303 & 195 & 118,004 & 130 & 119,383 & 325 \\
\hline Assistant Director & 101,983 & 92 & 113,257 & 76 & 107,083 & 168 \\
\hline Head, Branch & 80,695 & 299 & 84,752 & 153 & 82,068 & 452 \\
\hline Functional Specialist & 63,847 & 1,131 & 65,981 & 1,007 & 64,852 & 2,138 \\
\hline Subject Specialist & 65,576 & 710 & 69,439 & 439 & 67,052 & 1,149 \\
\hline \multicolumn{7}{|l|}{ Dept. Head: } \\
\hline Acquisitions & 76,056 & 82 & 75,563 & 30 & 75,924 & 112 \\
\hline Reference & 80,467 & 83 & 84,979 & 28 & 81,605 & 111 \\
\hline Cataloging & 76,903 & 113 & 75,455 & 41 & 76,517 & 154 \\
\hline Serials & 78,196 & 20 & 68,943 & 5 & 76,346 & 25 \\
\hline Documents/Maps & 71,437 & 34 & 72,960 & 23 & 72,052 & 57 \\
\hline Circulation & 74,686 & 56 & 65,272 & 28 & 71,548 & 84 \\
\hline Rare Books/Manuscripts & 83,595 & 44 & 83,382 & 46 & 83,486 & 90 \\
\hline Computer Systems & 95,783 & 26 & 90,132 & 44 & 92,231 & 70 \\
\hline Other & 78,011 & 399 & 80,295 & 236 & 78,860 & 635 \\
\hline \multicolumn{7}{|l|}{ Reference: } \\
\hline Over 14 years experience & 70,864 & 404 & 69,599 & 169 & 70,491 & 573 \\
\hline 10 to 14 years experience & 61,275 & 145 & 62,508 & 63 & 61,649 & 208 \\
\hline 5 to 9 years experience & 56,109 & 206 & 57,283 & 74 & 56,420 & 280 \\
\hline Under 5 years experience & 52,470 & 152 & 51,494 & 55 & 52,211 & 207 \\
\hline \multicolumn{7}{|l|}{ Cataloging: } \\
\hline Over 14 years experience & 66,713 & 263 & 68,155 & 125 & 67,178 & 388 \\
\hline 10 to 14 years experience & 58,373 & 73 & 62,105 & 31 & 59,485 & 104 \\
\hline 5 to 9 years experience & 55,073 & 99 & 57,803 & 38 & 55,830 & 137 \\
\hline Under 5 years experience & 50,793 & 66 & 50,748 & 31 & 50,778 & 97 \\
\hline \multicolumn{7}{|l|}{ Other: } \\
\hline Over 14 years experience & 70,402 & 203 & 70,642 & 70 & 70,464 & 273 \\
\hline 10 to 14 years experience & 61,901 & 86 & 60,214 & 29 & 61,476 & 115 \\
\hline 5 to 9 years experience & 53,449 & 94 & 59,749 & 38 & 55,263 & 132 \\
\hline Under 5 years experience & 51,654 & 97 & 51,210 & 38 & 51,529 & 135 \\
\hline All Positions & $\$ 71,230$ & 5,238 & $\$ 74,159$ & 3,091 & $\$ 72,317$ & 8,329 \\
\hline
\end{tabular}

Excludes medical and law libraries. See Tables 39 and 46 for comparable figures for medical and law libraries.

Canadian salaries are expressed in US dollars. See Table 32 for salaries of Canadian librarians expressed in Canadian dollars. 
Table 18: Number and Average Years of Experience of ARL University Librarians by Position And Sex, FY 2010-2011

\begin{tabular}{|c|c|c|c|c|c|c|}
\hline \multirow[b]{2}{*}{ Position } & \multicolumn{2}{|c|}{ WOMEN } & \multicolumn{2}{|c|}{ Men } & \multicolumn{2}{|c|}{ TOTAL } \\
\hline & Years & No. & Years & No. & Years & No. \\
\hline Director & 33.9 & 66 & 33.2 & 44 & 33.6 & 110 \\
\hline Associate Director & 26.5 & 195 & 24.3 & 130 & 25.6 & 325 \\
\hline Assistant Director & 25.2 & 92 & 22.9 & 76 & 24.2 & 168 \\
\hline Head, Branch & 21.8 & 299 & 23.7 & 153 & 22.4 & 452 \\
\hline Functional Specialist & 13.9 & 1,131 & 13.9 & 1,007 & 13.9 & 2,138 \\
\hline Subject Specialist & 16.0 & 710 & 17.5 & 439 & 16.6 & 1,149 \\
\hline \multicolumn{7}{|l|}{ Dept. Head: } \\
\hline Acquisitions & 22.0 & 82 & 18.8 & 30 & 21.2 & 112 \\
\hline Reference & 20.3 & 83 & 22.3 & 28 & 20.8 & 111 \\
\hline Cataloging & 21.5 & 113 & 22.3 & 41 & 21.7 & 154 \\
\hline Serials & 21.2 & 20 & 18.0 & 5 & 20.6 & 25 \\
\hline Documents/Maps & 21.7 & 34 & 21.2 & 23 & 21.5 & 57 \\
\hline Circulation & 19.7 & 56 & 18.1 & 28 & 19.2 & 84 \\
\hline Rare Books/Manuscripts & 21.0 & 44 & 23.8 & 46 & 22.4 & 90 \\
\hline Computer Systems & 21.8 & 26 & 17.4 & 44 & 19.0 & 70 \\
\hline Other & 20.4 & 399 & 19.1 & 236 & 19.9 & 635 \\
\hline Public services & 13.5 & 230 & 12.0 & 77 & 13.1 & 307 \\
\hline Technical services & 15.7 & 121 & 14.2 & 47 & 15.3 & 168 \\
\hline Administration & 15.7 & 129 & 14.7 & 51 & 15.5 & 180 \\
\hline Reference & 15.3 & 907 & 15.2 & 361 & 15.3 & 1,268 \\
\hline Cataloger & 17.4 & 501 & 18.1 & 225 & 17.6 & 726 \\
\hline All Positions & 17.3 & 5,238 & 17.2 & 3,091 & 17.3 & 8,329 \\
\hline
\end{tabular}

Excludes medical and law libraries. See Tables 40 and 47 for comparable figures for medical and law libraries.

Canadian salaries are expressed in US dollars. See Table 33 for salaries of Canadian librarians expressed in Canadian dollars. 
Table 19: Number and Average Salaries of ARL University Librarians by Years of Experience AND Sex, FY 2010-2011

\begin{tabular}{|c|c|c|c|c|c|c|c|}
\hline \multirow[b]{2}{*}{ Position } & \multicolumn{2}{|c|}{ WoMEN } & \multicolumn{2}{|c|}{ Men } & \multicolumn{2}{|c|}{ Total } & \multirow{2}{*}{$\begin{array}{c}\% \text { OF } \\
\text { TotAL }\end{array}$} \\
\hline & Salary & No. & Salary & No. & Salary & No. & \\
\hline $0-3$ years & $\$ 52,119$ & 510 & $\$ 53,359$ & 281 & $\$ 52,559$ & 791 & $9 \%$ \\
\hline 4-7 years & 55,553 & 754 & 57,412 & 408 & 56,206 & 1,162 & $14 \%$ \\
\hline 8-11 years & 62,306 & 725 & 65,184 & 467 & 63,433 & 1,192 & $14 \%$ \\
\hline $12-15$ years & 67,850 & 632 & 70,634 & 405 & 68,937 & 1,037 & $12 \%$ \\
\hline 16-19 years & 71,752 & 473 & 75,951 & 302 & 73,388 & 775 & $9 \%$ \\
\hline 20-23 years & 76,649 & 560 & 77,362 & 319 & 76,908 & 879 & $11 \%$ \\
\hline 24-27 years & 80,829 & 442 & 85,297 & 257 & 82,472 & 699 & $8 \%$ \\
\hline 28-31 years & 86,385 & 416 & 87,248 & 245 & 86,705 & 661 & $8 \%$ \\
\hline 32-35 years & 90,264 & 347 & 100,108 & 222 & 94,105 & 569 & $7 \%$ \\
\hline over 35 years & 96,923 & 379 & 100,671 & 185 & 98,153 & 564 & $7 \%$ \\
\hline All Positions & $\$ 71,230$ & 5,238 & $\$ 74,159$ & 3,091 & $\$ 72,317$ & 8,329 & $100 \%$ \\
\hline
\end{tabular}

Excludes medical and law libraries. See Tables 41 and 48 for comparable figures for medical and law libraries.

Canadian salaries are expressed in US dollars. See Table 34 for salaries of Canadian librarians expressed in Canadian dollars. 


\section{Table 20: Average Salaries of ARL University Librarians}

\section{by Position and Years of Experience, FY 2010-2011}

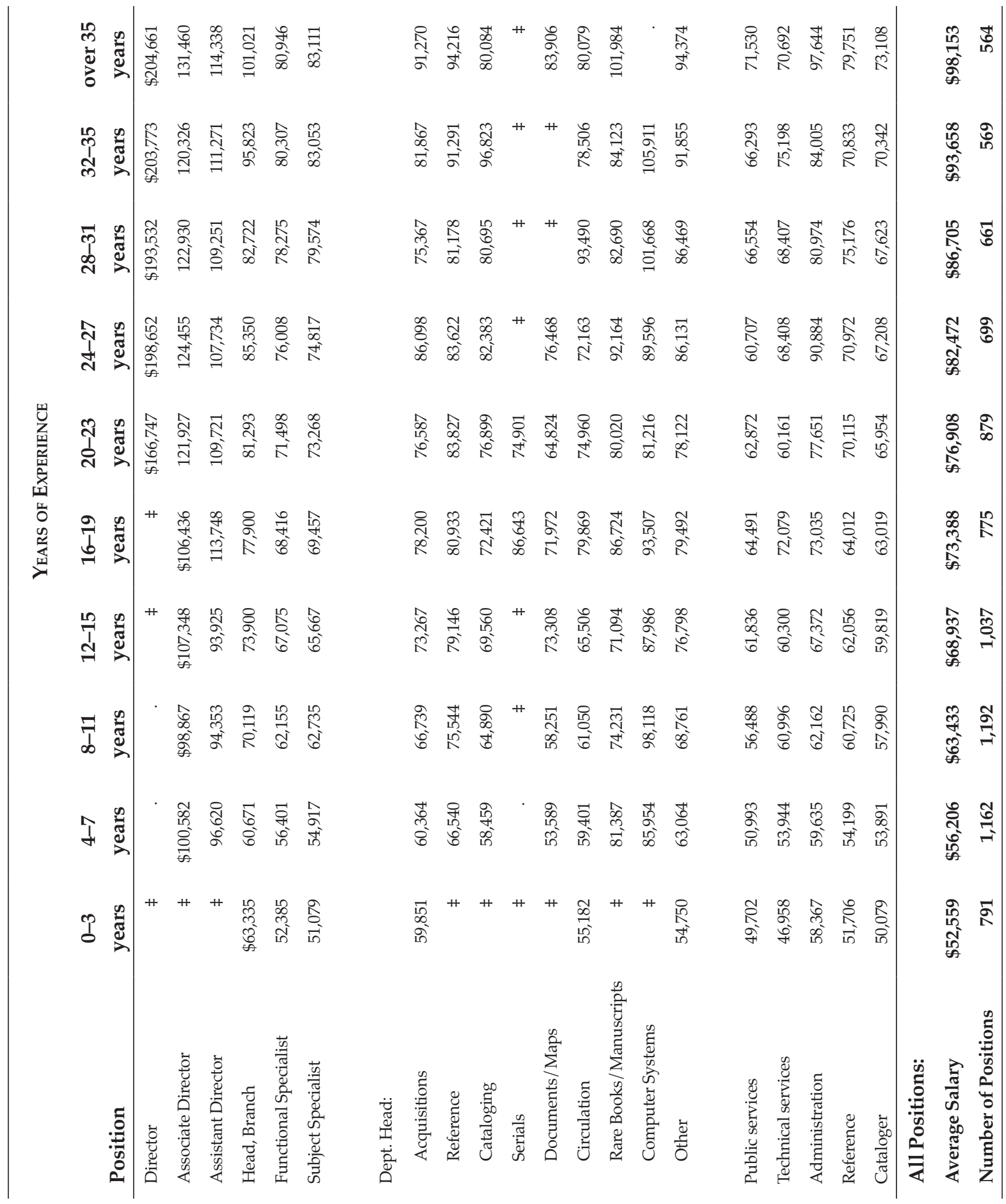

Years of experience reflect total professional experience.

Excludes medical and law libraries.

Canadian salaries are expressed in US dollars.

‡ Salary data are not published when fewer than four individuals are involved.

. No positions reported in this category. 
Table 21: Number and Average Salaries of ARL University Librarians by Position AND TyPe OF Institution, FY 2010-2011

\begin{tabular}{|c|c|c|c|c|c|c|c|c|}
\hline \multirow[b]{2}{*}{ Position } & \multicolumn{2}{|c|}{ CANADIAN (16) } & \multicolumn{2}{|c|}{ Private (31) } & \multicolumn{2}{|c|}{ Public (68) } & \multicolumn{2}{|c|}{ Total (115) } \\
\hline & Salary & No. & Salary & No. & Salary & No. & Salary & No. \\
\hline Director & $\$ 167,242$ & 16 & $\$ 236,090$ & 29 & $\$ 193,933$ & 65 & $\$ 201,165$ & 110 \\
\hline Associate Director & 121,022 & 46 & 130,596 & 92 & 113,464 & 187 & 119,383 & 325 \\
\hline Assistant Director & 102,515 & 17 & 108,861 & 79 & 106,211 & 72 & 107,083 & 168 \\
\hline Head, Branch & 97,517 & 62 & 84,910 & 112 & 77,478 & 278 & 82,068 & 452 \\
\hline Functional Specialist & 75,411 & 167 & 68,188 & 831 & 60,873 & 1,140 & 64,852 & 2,138 \\
\hline Subject Specialist & 71,887 & 130 & 67,890 & 398 & 65,502 & 621 & 67,052 & 1,149 \\
\hline \multicolumn{9}{|l|}{ Dept. Head: } \\
\hline Acquisitions & 91,481 & 16 & 74,494 & 34 & 72,694 & 62 & 75,924 & 112 \\
\hline Reference & 90,489 & 12 & 82,901 & 42 & 78,779 & 57 & 81,605 & 111 \\
\hline Cataloging & 92,377 & 12 & 78,882 & 59 & 72,543 & 83 & 76,517 & 154 \\
\hline Serials & 92,313 & 4 & 73,269 & 11 & 73,342 & 10 & 76,346 & 25 \\
\hline Documents/Maps & 96,019 & 9 & 69,386 & 16 & 66,644 & 32 & 72,052 & 57 \\
\hline Circulation & 78,883 & 14 & 72,519 & 22 & 68,964 & 48 & 71,548 & 84 \\
\hline Rare Books/Manuscripts & 91,750 & 7 & 82,873 & 32 & 82,736 & 51 & 83,486 & 90 \\
\hline Computer Systems & 94,836 & 9 & 91,124 & 18 & 92,149 & 43 & 92,231 & 70 \\
\hline Other & 91,453 & 68 & 80,968 & 177 & 75,707 & 390 & 78,860 & 635 \\
\hline \multicolumn{9}{|l|}{ Reference: } \\
\hline Over 14 years experience & 93,383 & 93 & 66,581 & 138 & 65,843 & 342 & 70,491 & 573 \\
\hline 10 to 14 years experience & 75,721 & 38 & 61,144 & 59 & 57,099 & 111 & 61,649 & 208 \\
\hline 5 to 9 years experience & 69,092 & 63 & 56,169 & 60 & 51,430 & 157 & 56,420 & 280 \\
\hline Under 5 years experience & 62,739 & 59 & 50,562 & 43 & 46,970 & 105 & 52,211 & 207 \\
\hline \multicolumn{9}{|l|}{ Cataloging: } \\
\hline Over 14 years experience & 82,259 & 34 & 67,321 & 155 & 64,490 & 199 & 67,178 & 388 \\
\hline 10 to 14 years experience & 71,448 & 7 & 61,653 & 50 & 55,397 & 47 & 59,485 & 104 \\
\hline 5 to 9 years experience & 70,778 & 12 & 58,227 & 63 & 50,502 & 62 & 55,830 & 137 \\
\hline Under 5 years experience & 66,359 & 9 & 51,810 & 45 & 46,437 & 43 & 50,778 & 97 \\
\hline \multicolumn{9}{|l|}{ Other: } \\
\hline Over 14 years experience & 82,045 & 20 & 71,863 & 81 & 68,458 & 172 & 70,464 & 273 \\
\hline 10 to 14 years experience & 69,831 & 12 & 68,269 & 28 & 57,603 & 75 & 61,476 & 115 \\
\hline 5 to 9 years experience & 68,077 & 7 & 61,578 & 42 & 50,986 & 83 & 55,263 & 132 \\
\hline Under 5 years experience & 59,992 & 14 & 55,542 & 42 & 47,896 & 79 & 51,529 & 135 \\
\hline All Positions & $\$ 83,424$ & 957 & $\$ 73,955$ & 2,758 & $\$ 69,034$ & 4,614 & $\$ 72,317$ & 8,329 \\
\hline
\end{tabular}

Excludes medical and law libraries.

Canadian salaries are expressed in US dollars. See Tables 31-34 for salaries of Canadian librarians expressed in Canadian dollars.

( ) Indicates the number of ARL libraries in each category. 
TABLE 22: Years of ExPERIENCE OF ARL University LibraRians

by Position and Type of Institution, FY 2010-2011

\begin{tabular}{|c|c|c|c|c|c|c|c|c|}
\hline \multirow[b]{2}{*}{ Position } & \multicolumn{2}{|c|}{ CANADIAN (16) } & \multicolumn{2}{|c|}{ Private (31) } & \multicolumn{2}{|c|}{ Public (68) } & \multicolumn{2}{|c|}{ Total (115) } \\
\hline & Years & No. & Years & No. & Years & No. & Years & No. \\
\hline Director & 32.6 & 16 & 33.5 & 29 & 34.0 & 65 & 33.6 & 110 \\
\hline Associate Director & 23.7 & 46 & 26.3 & 92 & 25.7 & 187 & 25.6 & 325 \\
\hline Assistant Director & 23.7 & 17 & 23.7 & 79 & 24.8 & 72 & 24.2 & 168 \\
\hline Head, Branch & 20.8 & 62 & 22.9 & 112 & 22.6 & 278 & 22.4 & 452 \\
\hline Functional Specialist & 14.5 & 167 & 14.0 & 831 & 13.8 & 1,140 & 13.9 & 2,138 \\
\hline Subject Specialist & 13.9 & 130 & 17.3 & 398 & 16.7 & 621 & 16.6 & 1,149 \\
\hline \multicolumn{9}{|l|}{ Dept. Head: } \\
\hline Acquisitions & 19.1 & 16 & 20.3 & 34 & 22.2 & 62 & 21.2 & 112 \\
\hline Reference & 18.1 & 12 & 20.9 & 42 & 21.3 & 57 & 20.8 & 111 \\
\hline Cataloging & 25.4 & 12 & 21.4 & 59 & 21.4 & 83 & 21.7 & 154 \\
\hline Serials & 22.8 & 4 & 21.2 & 11 & 19.0 & 10 & 20.6 & 25 \\
\hline Documents/Maps & 24.6 & 9 & 21.3 & 16 & 20.8 & 32 & 21.5 & 57 \\
\hline Circulation & 19.1 & 14 & 18.4 & 22 & 19.5 & 48 & 19.2 & 84 \\
\hline Rare Books/Manuscripts & 23.3 & 7 & 20.6 & 32 & 23.5 & 51 & 22.4 & 90 \\
\hline Computer Systems & 19.2 & 9 & 16.8 & 18 & 19.9 & 43 & 19.0 & 70 \\
\hline Other & 19.7 & 68 & 20.7 & 177 & 19.6 & 390 & 19.9 & 635 \\
\hline \multicolumn{9}{|l|}{ Reference: } \\
\hline Over 14 years experience & 25.7 & 93 & 26.1 & 138 & 24.9 & 342 & 25.3 & 573 \\
\hline 10 to 14 years experience & 11.4 & 38 & 11.9 & 59 & 11.5 & 111 & 11.6 & 208 \\
\hline 5 to 9 years experience & 7.0 & 63 & 7.0 & 60 & 6.9 & 157 & 6.9 & 280 \\
\hline Under 5 years experience & 2.3 & 59 & 2.7 & 43 & 2.6 & 105 & 2.5 & 207 \\
\hline \multicolumn{9}{|l|}{ Cataloging: } \\
\hline Over 14 years experience & 29.8 & 34 & 26.1 & 155 & 26.4 & 199 & 26.6 & 388 \\
\hline 10 to 14 years experience & 12.9 & 7 & 11.6 & 50 & 12.1 & 47 & 11.9 & 104 \\
\hline 5 to 9 years experience & 7.3 & 12 & 7.0 & 63 & 7.1 & 62 & 7.1 & 137 \\
\hline Under 5 years experience & 2.1 & 9 & 2.6 & 45 & 3.0 & 43 & 2.7 & 97 \\
\hline \multicolumn{9}{|l|}{ Other: } \\
\hline Over 14 years experience & 24.0 & 20 & 25.3 & 81 & 24.7 & 172 & 24.8 & 273 \\
\hline 10 to 14 years experience & 10.9 & 12 & 11.9 & 28 & 11.8 & 75 & 11.8 & 115 \\
\hline 5 to 9 years experience & 7.1 & 7 & 6.7 & 42 & 6.8 & 83 & 6.8 & 132 \\
\hline Under 5 years experience & 2.8 & 14 & 2.5 & 42 & 2.8 & 79 & 2.7 & 135 \\
\hline All Positions & 16.6 & 957 & 17.2 & 2,758 & 17.4 & 4,614 & 17.3 & 8,329 \\
\hline
\end{tabular}

Excludes medical and law libraries.

( ) Indicates the number of ARL libraries in each category. 


\section{Table 23: Number and Average Salaries of ARL University Librarians by Position and Size of Professional Staff, FY 2010-2011}

\begin{tabular}{|c|c|c|c|c|c|c|c|c|}
\hline \multirow[b]{2}{*}{ Position } & \multicolumn{2}{|c|}{$\begin{array}{c}\text { StafF OVER } 110 \\
\mathbf{( 1 5 )}^{+}\end{array}$} & \multicolumn{2}{|c|}{$\begin{array}{c}\text { STAFF 75-110 } \\
(\mathbf{1 7 )}\end{array}$} & \multicolumn{2}{|c|}{$\begin{array}{c}\text { STAFF } 50-74 \\
(48)\end{array}$} & \multicolumn{2}{|c|}{$\begin{array}{c}\text { STAFF 13-49 } \\
(35)^{\ddagger}\end{array}$} \\
\hline & Salary & No. & Salary & No. & Salary & No. & Salary & No. \\
\hline Director & $\$ 244,844$ & 14 & $\$ 222,538$ & 17 & $\$ 195,873$ & 46 & $\$ 178,999$ & 33 \\
\hline Associate Director & 136,680 & 65 & 121,478 & 72 & 113,583 & 119 & 110,906 & 69 \\
\hline Assistant Director & 119,265 & 38 & 102,412 & 40 & 105,584 & 71 & 98,156 & 19 \\
\hline Head, Branch & 85,564 & 135 & 83,668 & 78 & 80,370 & 169 & 77,643 & 70 \\
\hline Functional Specialist & 67,381 & 749 & 67,980 & 416 & 61,896 & 710 & 60,680 & 263 \\
\hline Subject Specialist & 68,842 & 349 & 70,983 & 231 & 65,264 & 469 & 60,107 & 100 \\
\hline \multicolumn{9}{|l|}{ Dept. Head: } \\
\hline Acquisitions & 74,776 & 20 & 86,294 & 16 & 73,829 & 48 & 74,410 & 28 \\
\hline Reference & 91,147 & 21 & 83,027 & 23 & 77,032 & 35 & 79,322 & 32 \\
\hline Cataloging & 83,135 & 46 & 80,891 & 23 & 70,335 & 54 & 74,221 & 31 \\
\hline Serials & 80,443 & 7 & 71,642 & 2 & 78,756 & 9 & 70,493 & 7 \\
\hline Documents/Maps & 81,922 & 10 & 67,847 & 9 & 74,261 & 20 & 66,215 & 18 \\
\hline Circulation & 81,209 & 18 & 74,503 & 13 & 68,097 & 36 & 66,368 & 17 \\
\hline Rare Books/Manuscripts & 95,439 & 11 & 90,010 & 18 & 77,934 & 33 & 81,139 & 28 \\
\hline Computer Systems & 109,147 & 10 & 106,409 & 9 & 85,048 & 33 & 88,912 & 18 \\
\hline Other & 83,885 & 162 & 80,971 & 116 & 76,401 & 252 & 74,674 & 105 \\
\hline Reference: & 70,791 & 109 & 69,528 & 85 & 71,070 & 209 & 70,068 & 170 \\
\hline \multicolumn{9}{|l|}{ Over 14 years experience } \\
\hline 10 to 14 years experience & 65,016 & 49 & 61,557 & 33 & 60,073 & 66 & 60,683 & 60 \\
\hline 5 to 9 years experience & 60,357 & 51 & 56,494 & 55 & 57,949 & 91 & 52,274 & 83 \\
\hline Under 5 years experience & 56,246 & 38 & 49,955 & 31 & 53,646 & 88 & 48,016 & 50 \\
\hline Cataloging: & 71,998 & 126 & 64,461 & 82 & 63,710 & 130 & 68,504 & 50 \\
\hline \multicolumn{9}{|l|}{ Over 14 years experience } \\
\hline 10 to 14 years experience & 63,613 & 44 & 58,514 & 20 & 56,612 & 26 & 53,236 & 14 \\
\hline 5 to 9 years experience & 60,065 & 51 & 55,542 & 27 & 52,290 & 40 & 52,328 & 19 \\
\hline Under 5 years experience & 55,209 & 32 & 47,245 & 25 & 51,637 & 27 & 44,883 & 13 \\
\hline Other: & 72,589 & 81 & 71,473 & 62 & 69,367 & 88 & 67,173 & 42 \\
\hline \multicolumn{9}{|l|}{ Over 14 years experience } \\
\hline 10 to 14 years experience & 65,835 & 42 & 59,654 & 33 & 61,601 & 24 & 53,603 & 16 \\
\hline 5 to 9 years experience & 55,556 & 46 & 58,054 & 25 & 55,761 & 36 & 51,215 & 25 \\
\hline Under 5 years experience & 53,512 & 52 & 54,252 & 25 & 50,153 & 40 & 45,079 & 18 \\
\hline All Positions & $\$ 73,863$ & 2,376 & $\$ 74,158$ & 1,586 & $\$ 71,110$ & 2,969 & $\$ 70,164$ & 1,398 \\
\hline
\end{tabular}

Excludes medical and law libraries.

Canadian salaries are expressed in US dollars. See Tables 31-34 for salaries of Canadian librarians expressed in Canadian dollars.

( ) Indicates the number of ARL libraries in each category.

† In 1995-1996 and earlier, the first column of this table reported staff over 124; in 1996-1998 over 120; in 1998-1999 over 115; and since 1999-2000, over 110.

‡ No ARL library has fewer than 13 professional staff members. 
TAble 24: Years of Experience of ARL University Librarians

by Position and Size of Professional Staff, FY 2010-2011

\begin{tabular}{|c|c|c|c|c|c|c|c|c|}
\hline \multirow[b]{2}{*}{ Position } & \multicolumn{2}{|c|}{$\begin{array}{c}\text { STAFF OVER } 110 \\
(\mathbf{1 5})^{+}\end{array}$} & \multicolumn{2}{|c|}{$\begin{array}{c}\text { STAFF 75-110 } \\
(\mathbf{1 7 )})\end{array}$} & \multicolumn{2}{|c|}{$\begin{array}{c}\text { STAFF 50-74 } \\
(48)\end{array}$} & \multicolumn{2}{|c|}{$\begin{array}{c}\text { STAFF 13-49 } \\
(35)^{\ddagger}\end{array}$} \\
\hline & Years & No. & Years & No. & Years & No. & Years & No. \\
\hline Director & 35.6 & 14 & 34.8 & 17 & 31.7 & 46 & 34.9 & 33 \\
\hline Associate Director & 25.8 & 65 & 26.4 & 72 & 24.9 & 119 & 25.8 & 69 \\
\hline Assistant Director & 25.4 & 38 & 22.2 & 40 & 24.7 & 71 & 24.1 & 19 \\
\hline Head, Branch & 23.0 & 135 & 22.8 & 78 & 21.6 & 169 & 22.8 & 70 \\
\hline Functional Specialist & 13.5 & 749 & 13.8 & 416 & 14.3 & 710 & 14.2 & 263 \\
\hline Subject Specialist & 15.9 & 349 & 17.0 & 231 & 17.4 & 469 & 14.2 & 100 \\
\hline \multicolumn{9}{|l|}{ Dept. Head: } \\
\hline Acquisitions & 20.7 & 20 & 23.2 & 16 & 20.6 & 48 & 21.4 & 28 \\
\hline Reference & 22.2 & 21 & 22.0 & 23 & 19.4 & 35 & 20.5 & 32 \\
\hline Cataloging & 22.9 & 46 & 22.9 & 23 & 21.6 & 54 & 19.3 & 31 \\
\hline Serials & 25.9 & 7 & 25.0 & 2 & 18.3 & 9 & 16.9 & 7 \\
\hline Documents/Maps & 22.4 & 10 & 18.8 & 9 & 25.8 & 20 & 17.6 & 18 \\
\hline Circulation & 21.7 & 18 & 18.8 & 13 & 18.4 & 36 & 18.3 & 17 \\
\hline Rare Books/Manuscripts & 20.5 & 11 & 23.9 & 18 & 21.1 & 33 & 23.8 & 28 \\
\hline Computer Systems & 21.9 & 10 & 16.9 & 9 & 17.8 & 33 & 20.6 & 18 \\
\hline Other & 21.8 & 162 & 19.1 & 116 & 19.1 & 252 & 19.8 & 105 \\
\hline \multicolumn{9}{|l|}{ Reference: } \\
\hline Over 14 years experience & 26.6 & 109 & 25.3 & 85 & 24.9 & 209 & 25.0 & 170 \\
\hline 10 to 14 years experience & 11.8 & 49 & 11.7 & 33 & 11.5 & 66 & 11.6 & 60 \\
\hline 5 to 9 years experience & 7.3 & 51 & 7.0 & 55 & 6.9 & 91 & 6.7 & 83 \\
\hline Under 5 years experience & 2.4 & 38 & 2.4 & 31 & 2.5 & 88 & 2.6 & 50 \\
\hline \multicolumn{9}{|l|}{ Cataloging: } \\
\hline Over 14 years experience & 27.0 & 126 & 24.4 & 82 & 27.5 & 130 & 26.6 & 50 \\
\hline 10 to 14 years experience & 11.6 & 44 & 11.7 & 20 & 12.3 & 26 & 12.2 & 14 \\
\hline 5 to 9 years experience & 7.0 & 51 & 7.0 & 27 & 6.9 & 40 & 7.8 & 19 \\
\hline Under 5 years experience & 3.0 & 32 & 2.3 & 25 & 2.7 & 27 & 2.8 & 13 \\
\hline \multicolumn{9}{|l|}{ Other: } \\
\hline Over 14 years experience & 24.9 & 81 & 25.2 & 62 & 24.4 & 88 & 24.9 & 42 \\
\hline 10 to 14 years experience & 11.7 & 42 & 11.7 & 33 & 11.9 & 24 & 11.8 & 16 \\
\hline 5 to 9 years experience & 6.6 & 46 & 6.6 & 25 & 6.8 & 36 & 7.2 & 25 \\
\hline Under 5 years experience & 2.7 & 52 & 2.6 & 25 & 2.9 & 40 & 2.4 & 18 \\
\hline All Positions & 16.8 & 2,376 & 17.1 & 1,586 & 17.6 & 2,969 & 17.6 & 1,398 \\
\hline
\end{tabular}

Excludes medical and law libraries.

( ) Indicates the number of ARL libraries in each category.

+ In 1995-1996 and earlier, the first column of this table reported staff over 124; in 1996-1998 over 120; in 1998-1999 over 115; and since 1999-2000, over 110.

‡ No ARL library has fewer than 13 professional staff members. 
Table 25: Average Salaries of ARL University Librarians by Position and Geographic Region, FY 2010-2011

\begin{tabular}{|c|c|c|c|c|c|c|c|}
\hline స్ّ & 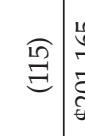 & 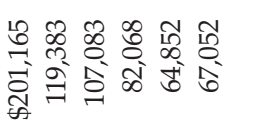 & 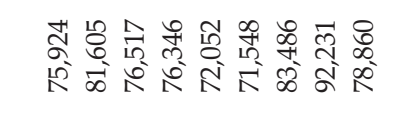 & 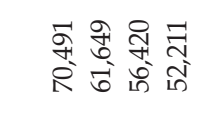 & 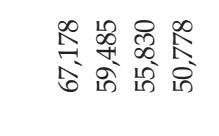 & 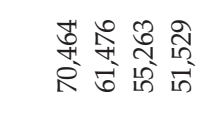 & 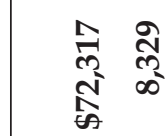 \\
\hline & 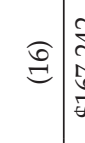 & 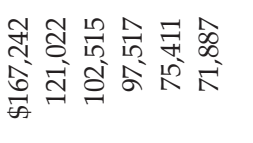 & 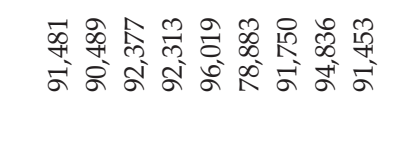 & 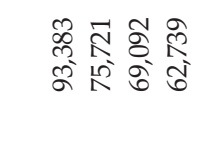 & 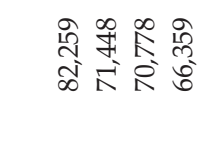 & 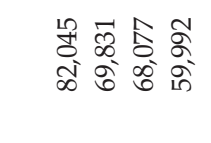 & 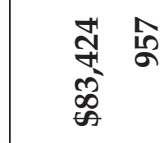 \\
\hline 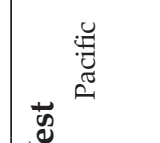 & $\widehat{\Xi} \mid \begin{array}{ll}\delta \\
\vdots \\
\delta \\
\vdots\end{array}$ & 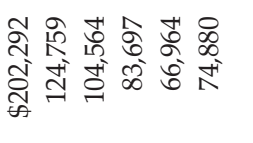 & 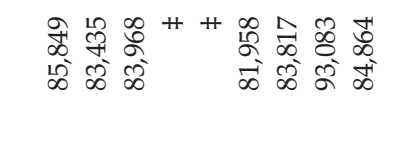 & 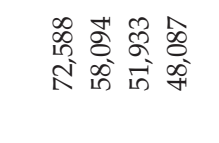 & 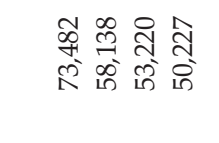 & 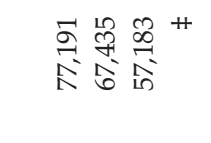 & 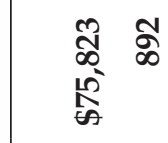 \\
\hline 胥 & $E$ & 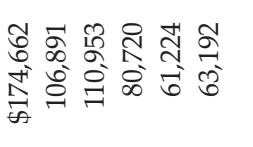 & 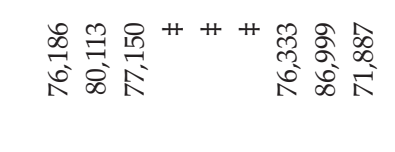 & $\begin{array}{l}\infty \\
\infty \\
\infty \\
\infty\end{array}$ & 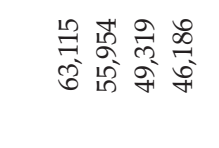 & 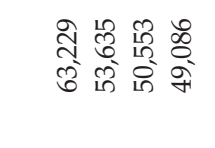 & 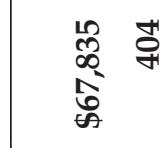 \\
\hline 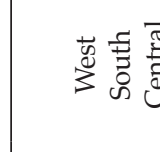 & 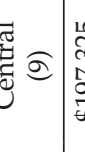 & 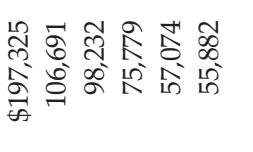 & 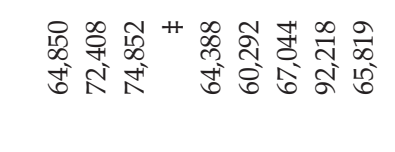 & 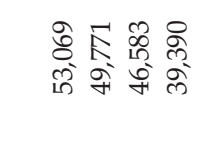 & 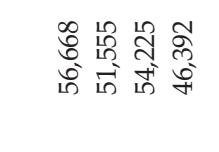 & 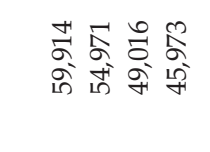 & 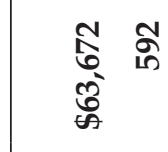 \\
\hline & 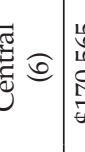 & 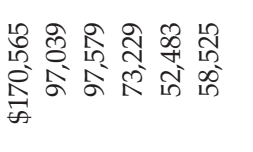 & 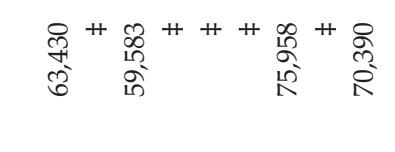 & 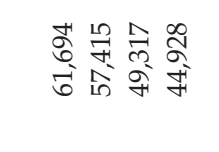 & 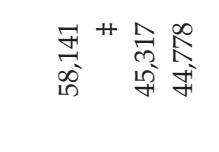 & 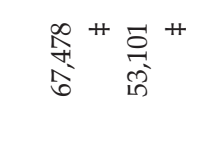 & 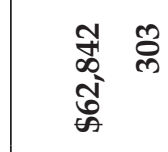 \\
\hline 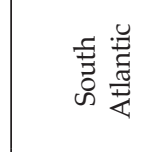 & 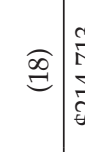 & 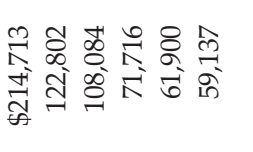 & 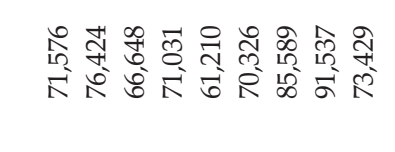 & 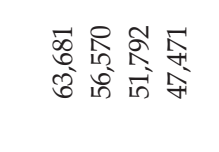 & 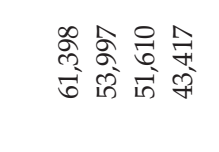 & 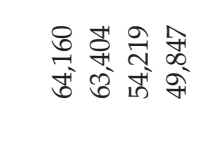 & 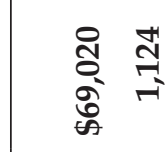 \\
\hline 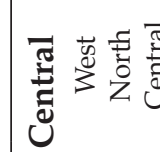 & $\left.\begin{array}{c}\vdots \\
\vdots\end{array}\right)$ & 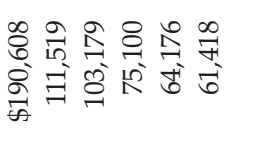 & 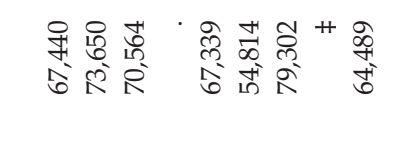 & 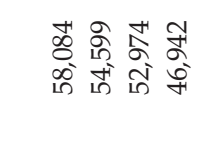 & 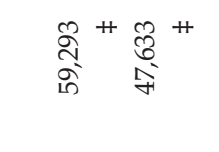 & 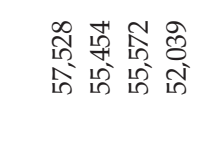 & 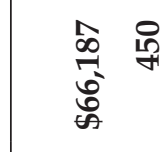 \\
\hline & 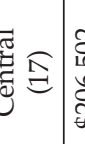 & 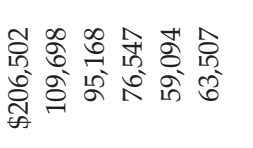 & 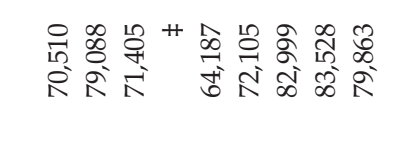 & 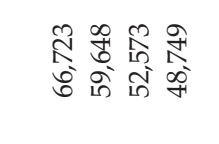 & 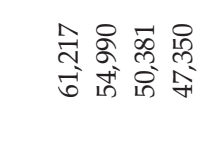 & 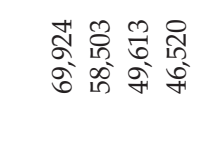 & 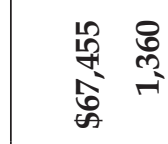 \\
\hline 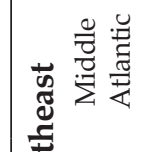 & $\mathscr{F}$ & 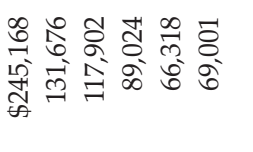 & 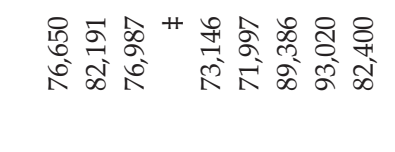 & 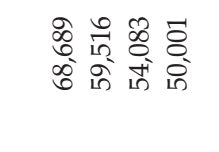 & 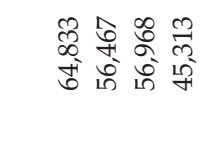 & 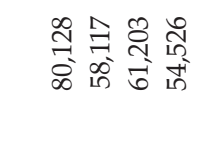 & 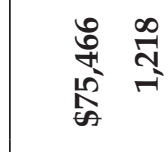 \\
\hline 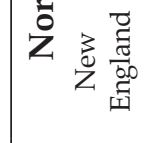 & 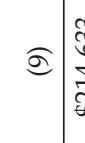 & 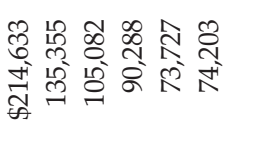 & 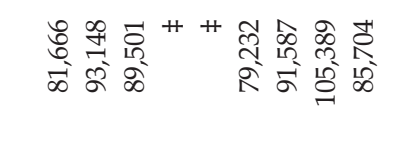 & 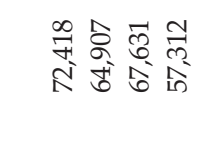 & 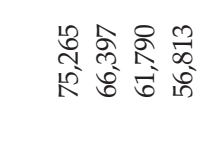 & 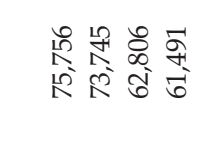 & 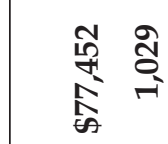 \\
\hline & 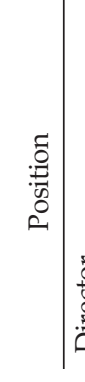 & 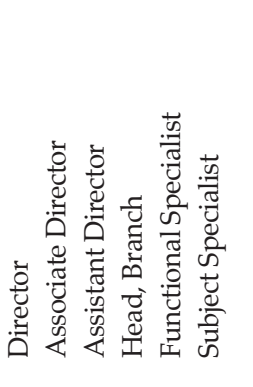 & 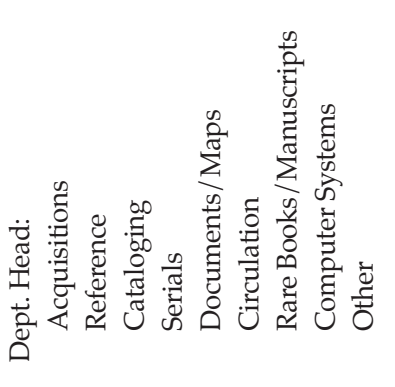 & 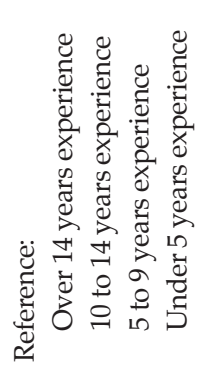 & 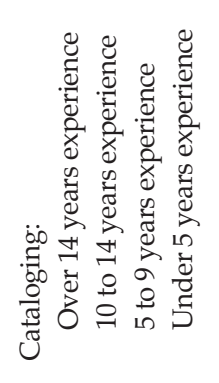 & 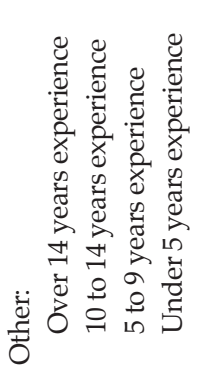 & 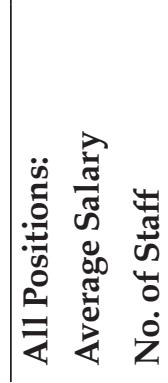 \\
\hline & & & . & & & & \\
\hline
\end{tabular}




\begin{tabular}{|c|c|}
\hline Region & $\begin{array}{l}\text { Number of } \\
\text { Libraries }\end{array}$ \\
\hline
\end{tabular}

\section{Northeast}

1. New England

2. Middle Atlantic

4. West North Central

\section{South}

5. South Atlantic

6. East South Central

7. West South Central

\section{West}

8. Mountain

9. Pacific

Canada
9 Boston University, Boston College, Brown, Connecticut, Dartmouth, Harvard, Massachusetts Institute of Technology, Massachusetts, Yale

14 Columbia; Cornell; New York; Pennsylvania; Pennsylvania State; Pittsburgh; Princeton; Rochester; Rutgers; State University of New York: Albany, Buffalo, Stony Brook; Syracuse; Temple

17 Case Western Reserve, Chicago, Cincinnati, IllinoisChicago, Illinois-Urbana, Indiana, Kent State, Michigan, Michigan State, Notre Dame, Northwestern, Ohio University, Ohio State, Purdue, Southern Illinois, Wayne State, Wisconsin

7 Iowa, Iowa State, Kansas, Minnesota, Missouri, Nebraska, Washington U.-St. Louis

18 Delaware, Duke, Emory, Florida, Florida State, Georgia, Georgia Tech., Georgetown, George Washington, Howard, Johns Hopkins, Maryland, Miami, North Carolina, North Carolina State, South Carolina, Virginia, Virginia Tech

6 Alabama, Auburn, Kentucky, Louisville, Tennessee, Vanderbilt

9 Houston, Louisiana State, Oklahoma, Oklahoma State, Rice, Texas, Texas A\&M, Texas Tech, Tulane

7 Arizona, Arizona State, Brigham Young, Colorado, Colorado State, New Mexico, Utah

University of California: Berkeley, Davis, Irvine, Los Angeles, Riverside, San Diego, Santa Barbara; Hawaii; Oregon; Southern California; Washington; Washington State

16 Alberta, British Columbia, Calgary, Guelph, Laval, McGill, McMaster, Manitoba, Montreal, Ottawa, Queen's, Saskatchewan, Toronto, Waterloo, Western Ontario, York
Conn., Mass., Me., N.H., R.I., Vt.

N.J., N.Y., Pa.

Ill., Ind., Mich., Ohio, Wis.

Iowa, Kan., Minn., Mo., Neb., N. Dak., S. Dak.

Del., DC, Fla., Ga., Md., N.C., S.C., Va., W. Va.

Ala., Ky., Miss., Tenn.

Ark., La., Okla., Tex.

Ariz., Colo., Idaho, Mont., Nev., N. Mex., Utah, Wyo.

Alaska, Calif., Hawaii, Ore., Wash.

Alta., B.C., Man., N. Br., Newf., N.S., Ont., P.E.I., Que., Sask.

Regions are based on the classification used by the US Bureau of the Census in tabulations of the Current Population Survey. 



\section{US ARL UNIVERSITY LIBRARIES}

TABles 26-30 
Table 26: Average Salaries of US ARL University Librarians by Position and Years of Experience, FY 2010-2011

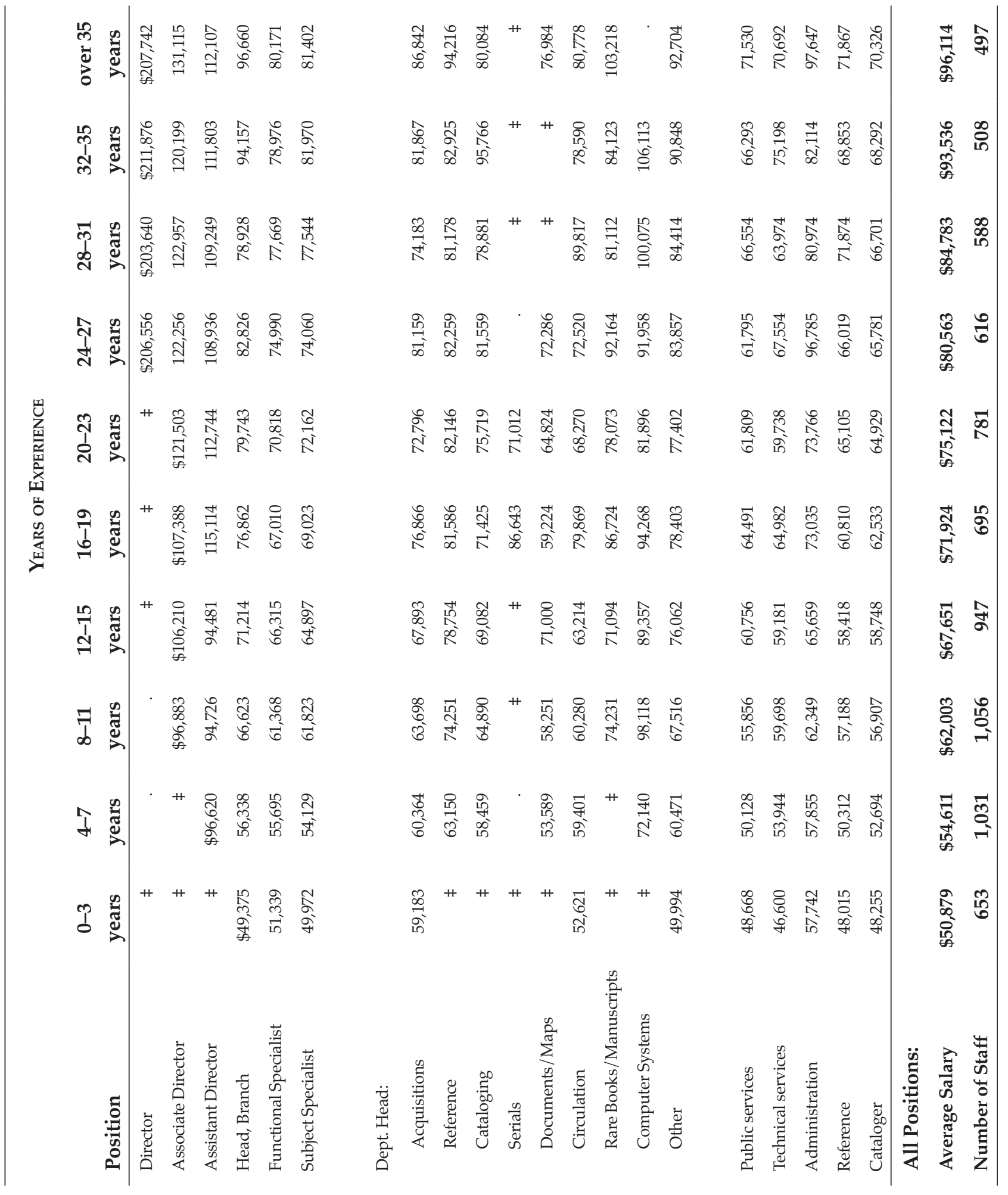

Excludes Canadian libraries.

Excludes medical and law libraries.

‡ Salary data are not published when fewer than four individuals are involved.

. No positions reported in this category. 


\begin{tabular}{|c|c|c|c|c|c|c|}
\hline \multirow[b]{2}{*}{ Position } & \multicolumn{2}{|c|}{ WOMEN } & \multicolumn{2}{|c|}{ Men } & \multicolumn{2}{|c|}{ Total } \\
\hline & Salary & No. & Salary & No. & Salary & No. \\
\hline Director & $\neq$ & 5 & $\neq$ & 2 & $\$ 167,134$ & 7 \\
\hline Associate Director & 121,755 & 17 & $\$ 123,541$ & 4 & 122,096 & 21 \\
\hline Assistant Director & 110,529 & 6 & 115,346 & 7 & 113,123 & 13 \\
\hline Head, Branch & 70,759 & 29 & 86,009 & 12 & 75,222 & 41 \\
\hline Functional Specialist & 60,905 & 145 & 64,933 & 122 & 62,745 & 267 \\
\hline Subject Specialist & 64,099 & 139 & 68,141 & 62 & 65,346 & 201 \\
\hline \multicolumn{7}{|l|}{ Dept. Head: } \\
\hline Acquisitions & $\neq$ & 8 & $\neq$ & 1 & 71,058 & 9 \\
\hline Reference & 79,622 & 8 & . & & 79,622 & 8 \\
\hline Cataloging & $\neq$ & 19 & $\neq$ & 2 & 72,608 & 21 \\
\hline Serials & $\neq$ & 1 & . & & $\neq$ & 1 \\
\hline Documents/Maps & $\neq$ & 3 & $\neq$ & 1 & 66,780 & 4 \\
\hline Circulation & $\neq$ & 2 & $\neq$ & 4 & 72,212 & 6 \\
\hline Rare Books/Manuscripts & $\neq$ & 5 & $\neq$ & 1 & 77,423 & 6 \\
\hline Computer Systems & 85,930 & 4 & 81,717 & 8 & 83,121 & 12 \\
\hline Other & 76,895 & 41 & 76,174 & 19 & 76,667 & 60 \\
\hline \multicolumn{7}{|l|}{ Reference: } \\
\hline Over 14 years experience & 69,520 & 44 & 67,587 & 16 & 69,005 & 60 \\
\hline 10 to 14 years experience & 56,684 & 9 & 58,217 & 7 & 57,355 & 16 \\
\hline 5 to 9 years experience & 53,539 & 30 & 57,871 & 8 & 54,451 & 38 \\
\hline Under 5 years experience & 47,094 & 25 & 49,822 & 6 & 47,622 & 31 \\
\hline \multicolumn{7}{|l|}{ Cataloging: } \\
\hline Over 14 years experience & 62,452 & 39 & 71,139 & 13 & 64,624 & 52 \\
\hline 10 to 14 years experience & 56,659 & 12 & 63,950 & 7 & 59,345 & 19 \\
\hline 5 to 9 years experience & 52,814 & 26 & 54,487 & 4 & 53,037 & 30 \\
\hline Under 5 years experience & $\neq$ & 15 & $\neq$ & 3 & 50,548 & 18 \\
\hline \multicolumn{7}{|l|}{ Other: } \\
\hline Over 14 years experience & 66,684 & 18 & 63,594 & 6 & 65,911 & 24 \\
\hline 10 to 14 years experience & $\neq$ & 8 & $\neq$ & 2 & 53,988 & 10 \\
\hline 5 to 9 years experience & $\neq$ & 16 & $\neq$ & 2 & 59,301 & 18 \\
\hline Under 5 years experience & $\neq$ & 20 & $\neq$ & 1 & 48,938 & 21 \\
\hline All Positions & $\$ 65,372$ & 694 & $\$ 69,565$ & 320 & $\$ 66,695$ & 1,014 \\
\hline
\end{tabular}

Excludes Canadian libraries.

Excludes medical and law libraries.

\# Salary data are not published when fewer than four individuals are involved in either category.

. No positions reported in this category. 
Table 28: Number and Average Years of Experience of Minority US ARL University Librarians by Position And Sex, FY 2010-2011

\begin{tabular}{|c|c|c|c|c|c|c|}
\hline \multirow[b]{2}{*}{ Position } & \multicolumn{2}{|c|}{ WOMEN } & \multicolumn{2}{|c|}{ Men } & \multicolumn{2}{|c|}{ Total } \\
\hline & Years & No. & Years & No. & Years & No. \\
\hline Director & 30.4 & 5 & 43.0 & 2 & 34.0 & 7 \\
\hline Associate Director & 28.9 & 17 & 21.5 & 4 & 27.5 & 21 \\
\hline Assistant Director & 24.7 & 6 & 16.0 & 7 & 20.0 & 13 \\
\hline Head, Branch & 21.0 & 29 & 21.1 & 12 & 21.0 & 41 \\
\hline Functional Specialist & 12.9 & 145 & 12.8 & 122 & 12.8 & 267 \\
\hline Subject Specialist & 14.1 & 139 & 14.1 & 62 & 14.1 & 201 \\
\hline \multicolumn{7}{|l|}{ Dept. Head: } \\
\hline Acquisitions & 14.4 & 8 & 4.0 & 1 & 13.2 & 9 \\
\hline Reference & 23.8 & 8 & & & 23.8 & 8 \\
\hline Cataloging & 20.4 & 19 & 24.5 & 2 & 20.8 & 21 \\
\hline Serials & 19.0 & 1 & & & 19.0 & 1 \\
\hline Documents/Maps & 12.3 & 3 & 13.0 & 1 & 12.5 & 4 \\
\hline Circulation & 8.5 & 2 & 22.8 & 4 & 18.0 & 6 \\
\hline Rare Books / Manuscripts & 16.6 & 5 & 18.0 & 1 & 16.8 & 6 \\
\hline Computer Systems & 18.8 & 4 & 14.1 & 8 & 15.7 & 12 \\
\hline Other & 17.6 & 41 & 17.6 & 19 & 17.6 & 60 \\
\hline Public services & 10.7 & 32 & 13.2 & 5 & 11.0 & 37 \\
\hline Technical services & 12.1 & 17 & & & 12.1 & 17 \\
\hline Administration & 13.1 & 13 & 13.8 & 6 & 13.3 & 19 \\
\hline Reference & 13.6 & 108 & 14.5 & 37 & 13.8 & 145 \\
\hline Cataloger & 14.7 & 92 & 17.1 & 27 & 15.2 & 119 \\
\hline All Positions & 15.0 & 694 & 14.8 & 320 & 14.9 & 1,014 \\
\hline
\end{tabular}

Excludes Canadian libraries. See Table 33 for comparable figures for Canadian libraries.

Excludes medical and law libraries. See Tables 40 and 47 for comparable figures for medical and law libraries.

. No positions reported in this category. 
Table 29: Number and Average Salaries of US ARL University Librarians by YeARs of EXPERIENCE AND SEX, FY 2010-2011

\begin{tabular}{|c|c|c|c|c|c|c|c|}
\hline \multirow[b]{2}{*}{ Position } & \multicolumn{2}{|c|}{ WOMEN } & \multicolumn{2}{|c|}{ MeN } & \multicolumn{2}{|c|}{ Total } & \multirow{2}{*}{$\begin{array}{l}\% \text { OF } \\
\text { TotaL }\end{array}$} \\
\hline & Salary & No. & Salary & No. & Salary & No. & \\
\hline $0-3$ years & $\$ 50,437$ & 416 & $\$ 51,655$ & 237 & $\$ 50,879$ & 653 & $9 \%$ \\
\hline $4-7$ years & 53,716 & 659 & 56,196 & 372 & 54,611 & 1,031 & $14 \%$ \\
\hline 8-11 years & 60,601 & 634 & 64,110 & 422 & 62,003 & 1,056 & $14 \%$ \\
\hline $12-15$ years & 66,239 & 571 & 69,796 & 376 & 67,651 & 947 & $13 \%$ \\
\hline $16-19$ years & 70,173 & 421 & 74,615 & 274 & 71,924 & 695 & $9 \%$ \\
\hline 20-23 years & 74,557 & 493 & 76,089 & 288 & 75,122 & 781 & $11 \%$ \\
\hline 24-27 years & 78,798 & 394 & 83,694 & 222 & 80,563 & 616 & $8 \%$ \\
\hline 28-31 years & 84,307 & 364 & 85,557 & 224 & 84,783 & 588 & $8 \%$ \\
\hline 32-35 years & 89,270 & 304 & 99,893 & 204 & 93,536 & 508 & $7 \%$ \\
\hline over 35 years & 94,710 & 331 & 98,914 & 166 & 96,114 & 497 & $7 \%$ \\
\hline All Positions & $\$ 69,576$ & 4,587 & $\$ 73,015$ & 2,785 & $\$ 70,875$ & 7,372 & $100 \%$ \\
\hline
\end{tabular}

Excludes Canadian libraries.

Excludes medical and law libraries. 
Table 30: Number and Average Salaries of Minority US ARL University Librarians by Years of EXPerience and SeX, FY 2010-2011

\begin{tabular}{|c|c|c|c|c|c|c|c|}
\hline \multirow[b]{2}{*}{ Position } & \multicolumn{2}{|c|}{ WOMEN } & \multicolumn{2}{|c|}{ Men } & \multicolumn{2}{|c|}{ TOTAL } & \multirow{2}{*}{$\begin{array}{l}\% \text { OF } \\
\text { TотаL }\end{array}$} \\
\hline & Salary & No. & Salary & No. & Salary & No. & \\
\hline $0-3$ years & $\$ 51,399$ & 91 & $\$ 55,956$ & 25 & $\$ 52,381$ & 116 & $11 \%$ \\
\hline 4-7 years & 54,481 & 131 & 56,676 & 58 & 55,155 & 189 & $19 \%$ \\
\hline 8-11 years & 59,618 & 96 & 67,962 & 71 & 63,165 & 167 & $16 \%$ \\
\hline 12-15 years & 66,708 & 81 & 68,946 & 52 & 67,583 & 133 & $13 \%$ \\
\hline 16-19 years & 67,519 & 77 & 76,328 & 27 & 69,806 & 104 & $10 \%$ \\
\hline 20-23 years & 76,128 & 76 & 74,583 & 23 & 75,769 & 99 & $10 \%$ \\
\hline 24-27 years & 74,361 & 41 & 77,830 & 15 & 75,290 & 56 & $6 \%$ \\
\hline 28-31 years & 73,047 & 34 & 77,017 & 21 & 74,563 & 55 & $5 \%$ \\
\hline 32-35 years & 84,712 & 28 & 83,823 & 12 & 84,445 & 40 & $4 \%$ \\
\hline over 35 years & 90,728 & 39 & 99,819 & 16 & 93,373 & 55 & $5 \%$ \\
\hline All Positions & $\$ 65,372$ & 694 & $\$ 69,565$ & 320 & $\$ 66,695$ & 1,014 & $100 \%$ \\
\hline
\end{tabular}

Excludes Canadian libraries.

Excludes medical and law libraries. 
CANAdian ARL University Libraries

TABles 31-34 
Table 31: Filled Positions; Average, Median, and Beginning Professional Salaries; and Average Years of Professional Experience in Canadian ARL University Libraries, FY 2010-2011

\begin{tabular}{|c|c|c|c|c|c|c|c|c|}
\hline \multirow[b]{2}{*}{ Institution } & \multirow{2}{*}{$\begin{array}{c}\text { FILLED } \\
\text { Positions } \\
\text { FY } 2011\end{array}$} & \multicolumn{2}{|c|}{ Average Salaries } & \multicolumn{2}{|c|}{ Median SAlaries } & \multicolumn{2}{|c|}{ Beginning Salaries } & \multirow{2}{*}{$\begin{array}{l}\text { AvERAGE } \\
\text { Yrs. ExP. } \\
\text { FY } 2011\end{array}$} \\
\hline & & FY 2010 & FY 2011 & FY 2010 & FY 2011 & FY 2010 & FY 2011 & \\
\hline Alberta $^{\ddagger}$ & 72 & $\$ 98,203$ & $\$ 101,564$ & $\$ 104,209$ & $\$ 102,139$ & $\$ 50,632$ & $\$ 55,770$ & 15.7 \\
\hline British Columbia ${ }^{\ddagger}$ & 72 & 85,196 & 89,757 & 81,675 & 87,753 & 55,335 & 55,335 & 17.7 \\
\hline Calgary ${ }^{\ddagger}$ & 53 & 94,823 & 96,879 & 89,543 & 93,666 & 58,000 & 58,000 & 18.7 \\
\hline Guelph ${ }^{\ddagger}$ & 50 & 80,634 & 85,069 & 78,054 & 81,146 & 59,699 & 61,639 & 18.1 \\
\hline Laval & 65 & 72,293 & 71,189 & 72,888 & 73,833 & 49,547 & 49,547 & 13.1 \\
\hline McGill & 64 & 74,432 & 75,422 & 67,475 & 68,471 & 50,000 & 50,000 & 15.7 \\
\hline McMaster $^{\ddagger}$ & 45 & 71,878 & 72,880 & 72,928 & 65,572 & 46,817 & 48,500 & 17.6 \\
\hline Manitoba $^{\ddagger}$ & 40 & 95,500 & 96,777 & 100,070 & 100,644 & 48,820 & 48,820 & 22.9 \\
\hline Montreal ${ }^{\ddagger}$ & 89 & 73,199 & 74,421 & 69,243 & 69,243 & 43,130 & 45,915 & 15.3 \\
\hline Ottawa $\ddagger^{*}$ & 34 & $\mathrm{~N} / \mathrm{A}$ & 92,217 & $\mathrm{~N} / \mathrm{A}$ & 89,855 & $\mathrm{~N} / \mathrm{A}$ & 50,181 & 18.9 \\
\hline Queen's & 32 & 93,924 & 101,504 & 97,348 & 104,510 & 51,600 & 53,251 & 21.3 \\
\hline Saskatchewan ${ }^{\ddagger}$ & 46 & 89,027 & 89,895 & 91,994 & 86,118 & 52,731 & 55,104 & 16.0 \\
\hline Toronto $^{\ddagger}$ & 141 & 91,249 & 90,332 & 87,729 & 86,827 & 51,900 & 52,200 & 15.6 \\
\hline Waterloo $^{\ddagger}$ & 36 & 81,938 & 80,906 & 81,972 & 80,198 & 51,804 & 51,840 & 17.4 \\
\hline Western Ontario ${ }^{\ddagger}$ & 65 & 68,921 & 72,394 & 65,014 & 70,684 & 47,380 & 50,496 & 13.4 \\
\hline York $^{\ddagger}$ & 53 & 96,016 & 105,317 & 90,210 & 101,808 & 49,000 & 49,000 & 17.6 \\
\hline
\end{tabular}


Table 32: Number and Average Salaries of Canadian ARL University Librarians by Position and Sex, FY 2010-2011

\begin{tabular}{|c|c|c|c|c|c|c|}
\hline \multirow[b]{2}{*}{ Position } & \multicolumn{2}{|c|}{ WOMEN } & \multicolumn{2}{|c|}{ MeN } & \multicolumn{2}{|c|}{ Total } \\
\hline & Salary & No. & Salary & No. & Salary & No. \\
\hline Director & $\$ 175,367$ & 9 & $\$ 178,049$ & 7 & $\$ 176,540$ & 16 \\
\hline Associate Director & 129,756 & 31 & 123,607 & 15 & 127,751 & 46 \\
\hline Assistant Director & 106,501 & 11 & 111,356 & 6 & 108,215 & 17 \\
\hline Head, Branch & 103,962 & 50 & 98,678 & 12 & 102,939 & 62 \\
\hline Functional Specialist & 77,459 & 82 & 81,673 & 85 & 79,604 & 167 \\
\hline Subject Specialist & 73,220 & 87 & 81,272 & 43 & 75,883 & 130 \\
\hline \multicolumn{7}{|l|}{ Dept. Head: } \\
\hline Acquisitions & 93,517 & 12 & 105,718 & 4 & 96,568 & 16 \\
\hline Reference & $\neq$ & 11 & $\neq$ & 1 & 95,521 & 12 \\
\hline Cataloging & $\neq$ & 10 & $\neq$ & 2 & 97,513 & 12 \\
\hline Serials & 97,445 & 4 & . & & 97,445 & 4 \\
\hline Documents/Maps & 105,266 & 5 & 96,472 & 4 & 101,357 & 9 \\
\hline Circulation & $\neq$ & 12 & $\neq$ & 2 & 83,269 & 14 \\
\hline Rare Books / Manuscripts & $\neq$ & 4 & $\neq$ & 3 & 96,851 & 7 \\
\hline Computer Systems & 104,481 & 4 & 96,611 & 5 & 100,109 & 9 \\
\hline Other & 96,866 & 47 & 95,805 & 21 & 96,538 & 68 \\
\hline \multicolumn{7}{|l|}{ Reference: } \\
\hline Over 14 years experience & 97,734 & 70 & 101,137 & 23 & 98,575 & 93 \\
\hline 10 to 14 years experience & 80,562 & 24 & 78,851 & 14 & 79,932 & 38 \\
\hline 5 to 9 years experience & 72,445 & 49 & 74,644 & 14 & 72,934 & 63 \\
\hline Under 5 years experience & 65,610 & 48 & 68,923 & 11 & 66,227 & 59 \\
\hline \multicolumn{7}{|l|}{ Cataloging: } \\
\hline Over 14 years experience & 87,762 & 22 & 85,128 & 12 & 86,832 & 34 \\
\hline 10 to 14 years experience & $\neq$ & 6 & $\neq$ & 1 & 75,420 & 7 \\
\hline 5 to 9 years experience & 77,599 & 8 & 68,943 & 4 & 74,714 & 12 \\
\hline Under 5 years experience & $\neq$ & 6 & $\neq$ & 3 & 70,048 & 9 \\
\hline \multicolumn{7}{|l|}{ Other: } \\
\hline Over 14 years experience & 86,571 & 14 & 86,691 & 6 & 86,607 & 20 \\
\hline 10 to 14 years experience & $\neq$ & 10 & $\neq$ & 2 & 73,714 & 12 \\
\hline 5 to 9 years experience & $\neq$ & 5 & $\neq$ & 2 & 71,862 & 7 \\
\hline Under 5 years experience & 63,916 & 10 & 61,856 & 4 & 63,327 & 14 \\
\hline All Positions & $\$ 87,490$ & 651 & $\$ 89,278$ & 306 & $\$ 88,062$ & 957 \\
\hline
\end{tabular}

Salaries are expressed in Canadian dollars.

Excludes Canadian medical and law libraries. See Tables 39 and 46 for comparable figures for medical and law libraries.

t Salary data are not published when fewer than four individuals are involved in either category.

. No positions reported in this category. 
Table 33: Number and Average Years of Experience of Canadian ARL University Librarians by Position ANd Sex, FY 2010-2011

\begin{tabular}{|c|c|c|c|c|c|c|}
\hline \multirow[b]{2}{*}{ Position } & \multicolumn{2}{|c|}{ WOMEN } & \multicolumn{2}{|c|}{ MeN } & \multicolumn{2}{|c|}{ Total } \\
\hline & Years & No. & Years & No. & Years & No. \\
\hline Director & 33.6 & 9 & 31.3 & 7 & 32.6 & 16 \\
\hline Associate Director & 24.3 & 31 & 22.3 & 15 & 23.7 & 46 \\
\hline Assistant Director & 23.5 & 11 & 24.2 & 6 & 23.7 & 17 \\
\hline Head, Branch & 21.1 & 50 & 19.7 & 12 & 20.8 & 62 \\
\hline Functional Specialist & 13.4 & 82 & 15.5 & 85 & 14.5 & 167 \\
\hline Subject Specialist & 13.4 & 87 & 14.8 & 43 & 13.9 & 130 \\
\hline \multicolumn{7}{|l|}{ Dept. Head: } \\
\hline Acquisitions & 18.1 & 12 & 22.0 & 4 & 19.1 & 16 \\
\hline Reference & 16.8 & 11 & 32.0 & 1 & 18.1 & 12 \\
\hline Cataloging & 25.4 & 10 & 25.5 & 2 & 25.4 & 12 \\
\hline Serials & 22.8 & 4 & & & 22.8 & 4 \\
\hline Documents/Maps & 30.4 & 5 & 17.3 & 4 & 24.6 & 9 \\
\hline Circulation & 21.3 & 12 & 6.5 & 2 & 19.1 & 14 \\
\hline Rare Books/Manuscripts & 18.3 & 4 & 30.0 & 3 & 23.3 & 7 \\
\hline Computer Systems & 23.3 & 4 & 16.0 & 5 & 19.2 & 9 \\
\hline Other & 21.0 & 47 & 17.0 & 21 & 19.7 & 68 \\
\hline Public services & 10.3 & 12 & 7.4 & 7 & 9.2 & 19 \\
\hline Technical services & 14.9 & 8 & 18.0 & 5 & 16.1 & 13 \\
\hline Administration & 15.1 & 19 & 14.5 & 2 & 15.0 & 21 \\
\hline Reference & 13.3 & 191 & 13.7 & 62 & 13.4 & 253 \\
\hline Cataloger & 19.0 & 42 & 20.5 & 20 & 19.5 & 62 \\
\hline All Positions & 16.6 & 651 & 16.7 & 306 & 16.6 & 957 \\
\hline
\end{tabular}

Excludes Canadian medical and law libraries. See Tables 40 and 47 for comparable figures for medical and law libraries. . No positions reported in this category. 
Table 34: Number and Average Salaries of Canadian ARL University Librarians by YEARS OF EXPERIENCE AND SEX, FY 2010-2011

\begin{tabular}{|c|c|c|c|c|c|c|c|}
\hline \multirow[b]{2}{*}{ Experience } & \multicolumn{2}{|c|}{ WOMEN } & \multicolumn{2}{|c|}{ MeN } & \multicolumn{2}{|c|}{ TOTAL } & \multirow{2}{*}{$\begin{array}{r}\% \text { OF } \\
\text { TOTAL }\end{array}$} \\
\hline & Salary & No. & Salary & No. & Salary & No. & \\
\hline $0-3$ years & $\$ 62,872$ & 94 & $\$ 66,013$ & 44 & $\$ 63,874$ & 138 & $14 \%$ \\
\hline $4-7$ years & 72,092 & 95 & 73,865 & 36 & 72,579 & 131 & $14 \%$ \\
\hline 8-11 years & 78,313 & 91 & 79,432 & 45 & 78,683 & 136 & $14 \%$ \\
\hline $12-15$ years & 87,546 & 61 & 86,036 & 29 & 87,060 & 90 & $9 \%$ \\
\hline 16-19 years & 89,235 & 52 & 93,976 & 28 & 90,894 & 80 & $8 \%$ \\
\hline 20-23 years & 97,164 & 67 & 94,142 & 31 & 96,208 & 98 & $10 \%$ \\
\hline 24-27 years & 102,916 & 48 & 100,768 & 35 & 102,011 & 83 & $9 \%$ \\
\hline 28-31 years & 106,541 & 52 & 111,132 & 21 & 107,862 & 73 & $8 \%$ \\
\hline 32-35 years & 102,699 & 43 & 108,245 & 18 & 104,335 & 61 & $6 \%$ \\
\hline over 35 years & 118,424 & 48 & 122,471 & 19 & 119,572 & 67 & $7 \%$ \\
\hline All Positions & $\$ 87,490$ & 651 & $\$ 89,278$ & 306 & $\$ 88,062$ & 957 & $100 \%$ \\
\hline
\end{tabular}

Salaries are expressed in Canadian dollars

Excludes Canadian medical and law libraries. See Tables 41 and 48 for comparable figures for medical and law libraries. 

ARL University Medical Libraries

TABLes 35-41 
Table 35: Filled Positions; Average, Median, and Beginning Salaries; and Average Years of EXPERIENCE IN ARL University MedicAl LibraRies, FY 2010-2011

\begin{tabular}{|c|c|c|c|c|c|}
\hline Institution & $\begin{array}{c}\text { Filled } \\
\text { Positions }\end{array}$ & $\begin{array}{c}\text { Average } \\
\text { Salary }\end{array}$ & $\begin{array}{c}\text { Median } \\
\text { Salary }\end{array}$ & $\begin{array}{l}\text { Beginning } \\
\text { Salary }\end{array}$ & $\begin{array}{c}\text { Average Yrs. } \\
\text { Exp. }\end{array}$ \\
\hline Alabama & 2 & $\neq$ & $\neq$ & $\$ 42,000$ & 17.0 \\
\hline Alberta & 6 & $\$ 87,808$ & $\$ 105,587$ & 52,833 & 16.7 \\
\hline Arizona & 16 & 63,668 & 58,651 & 50,857 & 20.9 \\
\hline Boston University & 12 & 55,599 & 50,142 & 43,000 & 8.0 \\
\hline British Columbia & 12 & 79,772 & 82,574 & 52,420 & 18.9 \\
\hline Calgary & 12 & 73,414 & 67,294 & 54,945 & 11.0 \\
\hline California, Davis & 7 & 72,319 & 64,560 & 46,164 & 22.9 \\
\hline California, Los Angeles & 11 & 82,699 & 81,876 & 46,164 & 22.5 \\
\hline California, San Diego & 9 & 69,359 & 72,345 & 46,164 & 13.7 \\
\hline Case Western Reserve & 7 & 67,306 & 71,055 & 35,000 & 27.9 \\
\hline Cincinnati & 14 & 61,133 & 50,688 & 42,000 & 22.3 \\
\hline Columbia & 10 & 70,545 & 73,803 & 52,000 & 15.7 \\
\hline Connecticut & 15 & 77,815 & 75,152 & 55,347 & 17.5 \\
\hline Cornell & 12 & 76,332 & 75,542 & 55,000 & 18.6 \\
\hline Dartmouth & 8 & 63,723 & 64,415 & 45,500 & 20.8 \\
\hline Duke & 20 & 58,074 & 55,164 & 40,000 & 16.2 \\
\hline Emory & 12 & 62,851 & 56,959 & 47,750 & 20.4 \\
\hline Florida & 14 & 54,305 & 48,746 & 42,000 & 13.4 \\
\hline Florida State & 5 & 51,041 & 52,299 & 42,000 & 14.0 \\
\hline George Washington & 14 & 66,900 & 67,542 & 42,000 & 15.6 \\
\hline Georgetown & 12 & 60,976 & 62,500 & 44,000 & 13.1 \\
\hline Harvard & 59 & 78,066 & 75,531 & 53,093 & 5.4 \\
\hline Hawaii & 3 & $\neq$ & $\neq$ & 45,000 & 17.7 \\
\hline Howard & 7 & 67,369 & 60,776 & 50,000 & 21.3 \\
\hline Illinois, Chicago & 22 & 59,275 & 55,697 & 47,000 & 14.2 \\
\hline Iowa & 9 & 58,790 & 52,470 & 41,000 & 16.2 \\
\hline Johns Hopkins & 22 & 69,218 & 66,656 & 47,700 & 16.5 \\
\hline Kansas & 11 & 50,383 & 47,370 & 43,000 & 13.3 \\
\hline Kentucky & 14 & 56,480 & 54,282 & 41,000 & 24.1 \\
\hline Louisiana State & 1 & $\neq$ & $\neq$ & 36,000 & 5.0 \\
\hline Louisville & 9 & 58,207 & 56,293 & 37,000 & 24.6 \\
\hline McGill & 10 & 72,707 & 70,071 & 47,366 & 17.8 \\
\hline McMaster & 8 & 68,521 & 76,034 & 45,945 & 13.1 \\
\hline Manitoba & 18 & 72,939 & 71,754 & 46,249 & 15.4 \\
\hline Miami & 19 & 53,211 & 51,461 & 47,000 & 7.3 \\
\hline Michigan & 19 & 60,267 & 57,296 & 42,000 & 13.5 \\
\hline Minnesota & 15 & 63,946 & 62,846 & 43,000 & 17.1 \\
\hline Missouri & 9 & 53,831 & 50,017 & 40,000 & 21.8 \\
\hline Montreal & 10 & 69,345 & 65,596 & 50,297 & 16.1 \\
\hline Nebraska & 15 & 62,725 & 57,200 & 45,000 & 19.9 \\
\hline New Mexico & 14 & 65,631 & 62,620 & 39,372 & 21.4 \\
\hline New York University & 35 & 67,132 & 62,700 & 50,000 & 11.9 \\
\hline
\end{tabular}

68 · ARL Annual Salary Survey 2010-2011 
Table 35: Filled Positions; Average, Median, and Beginning Salaries; and Average Years of EXPERIENCE IN ARL University MedicAl LibRARIES, FY 2010-2011

\begin{tabular}{|c|c|c|c|c|c|}
\hline Institution & $\begin{array}{c}\text { Filled } \\
\text { Positions }\end{array}$ & $\begin{array}{c}\text { Average } \\
\text { Salary }\end{array}$ & $\begin{array}{c}\text { Median } \\
\text { Salary }\end{array}$ & $\begin{array}{l}\text { Beginning } \\
\text { Salary }\end{array}$ & $\begin{array}{c}\text { Average Yrs. } \\
\text { Exp. }\end{array}$ \\
\hline North Carolina & 30 & 68,618 & 65,049 & 45,000 & 19.8 \\
\hline Northwestern & 17 & 65,507 & 66,073 & 41,000 & 16.9 \\
\hline Ohio State & 11 & 62,170 & 59,919 & 43,243 & 15.9 \\
\hline Oklahoma & 9 & 59,817 & 60,019 & 40,000 & 19.9 \\
\hline Oklahoma State & 4 & $\neq$ & $\neq$ & 38,000 & 23.0 \\
\hline Ottawa & 6 & 78,426 & 73,277 & 47,538 & 17.8 \\
\hline Pennsylvania & 10 & 65,949 & 61,727 & 49,000 & 21.6 \\
\hline Pennsylvania State & 5 & 63,993 & 62,982 & 43,709 & 17.4 \\
\hline Pittsburgh & 25 & 62,379 & 60,227 & 45,000 & 16.2 \\
\hline Queen's & 8 & 86,981 & 85,492 & 50,446 & 17.8 \\
\hline Rochester & 25 & 56,577 & 53,001 & 38,983 & 20.2 \\
\hline Saskatchewan & 6 & 72,571 & 69,604 & 52,202 & 10.3 \\
\hline South Carolina & 9 & 49,291 & 46,692 & 35,000 & 14.3 \\
\hline Southern California & 14 & 72,947 & 71,300 & 50,000 & 17.9 \\
\hline Southern Illinois & 5 & 63,036 & 66,247 & 41,000 & 24.4 \\
\hline SUNY Buffalo & 14 & 68,258 & 65,833 & 47,000 & 21.4 \\
\hline SUNY Stony Brook & 19 & 70,087 & 71,317 & 45,000 & 15.1 \\
\hline Temple & 9 & 57,880 & 57,680 & 44,004 & 17.8 \\
\hline Tennessee, Knoxville & 4 & $\neq$ & $\neq$ & 40,000 & 14.0 \\
\hline Tennessee, Memphis & 11 & 53,636 & 51,091 & 45,000 & 26.1 \\
\hline Texas Tech & 23 & 49,315 & 45,634 & 38,110 & 19.5 \\
\hline Toronto & 13 & 88,333 & 89,289 & 49,451 & 17.4 \\
\hline Tulane & 14 & 40,606 & 38,684 & 40,000 & 13.0 \\
\hline Utah & 13 & 66,845 & 60,173 & 40,000 & 22.8 \\
\hline Vanderbilt & 13 & 63,781 & 67,060 & 41,000 & 17.3 \\
\hline Virginia & 14 & 65,115 & 65,700 & 45,000 & 22.3 \\
\hline Washington & 20 & 63,846 & 59,040 & 42,600 & 19.9 \\
\hline Washington U.-St. Louis & 24 & 61,693 & 51,750 & 40,000 & 18.2 \\
\hline Wayne State & 4 & $\neq$ & $\neq$ & 45,000 & 19.3 \\
\hline Wisconsin & 16 & 58,988 & 56,669 & 40,526 & 13.8 \\
\hline Yale & 20 & 76,505 & 75,556 & 50,500 & 17.6 \\
\hline
\end{tabular}

Directors are included in figures for filled positions and average years of experience, but not in either the average or median salary statistics. Canadian salaries are expressed in US dollars.

‡ Salary data are not published when fewer than four individuals are involved. 
Table 36: Beginning Professional Salaries in ARL University Medical Libraries

Rank Order Table, FY 2010-2011

\begin{tabular}{|c|c|c|c|c|c|}
\hline Rank & Institution & Salary & Rank & Institution & Salary \\
\hline 1 & Connecticut & 55,347 & 31 & Wayne State & 45,000 \\
\hline 2 & Cornell & 55,000 & 39 & Temple & 44,004 \\
\hline 3 & Calgary & 54,945 & 40 & Georgetown & 44,000 \\
\hline 4 & Harvard & 53,093 & 41 & Pennsylvania State & 43,709 \\
\hline 5 & Alberta & 52,833 & 42 & Ohio State & 43,243 \\
\hline 6 & British Columbia & 52,420 & 43 & Boston University & 43,000 \\
\hline 7 & Saskatchewan & 52,202 & 43 & Kansas & 43,000 \\
\hline 8 & Columbia & 52,000 & 43 & Minnesota & 43,000 \\
\hline 9 & Arizona & 50,857 & 46 & Washington & 42,600 \\
\hline 10 & Yale & 50,500 & 47 & Alabama & 42,000 \\
\hline 11 & Queen's & 50,446 & 47 & Cincinnati & 42,000 \\
\hline 12 & Montreal & 50,297 & 47 & Florida & 42,000 \\
\hline 13 & Howard & 50,000 & 47 & Florida State & 42,000 \\
\hline 13 & New York University & 50,000 & 47 & George Washington & 42,000 \\
\hline 13 & Southern California & 50,000 & 47 & Michigan & 42,000 \\
\hline 16 & Toronto & 49,451 & 53 & Iowa & 41,000 \\
\hline 17 & Pennsylvania & 49,000 & 53 & Kentucky & 41,000 \\
\hline 18 & Emory & 47,750 & 53 & Northwestern & 41,000 \\
\hline 19 & Johns Hopkins & 47,700 & 53 & Southern Illinois & 41,000 \\
\hline 20 & Ottawa & 47,538 & 53 & Vanderbilt & 41,000 \\
\hline 21 & McGill & 47,366 & 58 & Wisconsin & 40,526 \\
\hline 22 & Illinois, Chicago & 47,000 & 59 & Duke & 40,000 \\
\hline 22 & Miami & 47,000 & 59 & Missouri & 40,000 \\
\hline 22 & SUNY Buffalo & 47,000 & 59 & Oklahoma & 40,000 \\
\hline 25 & Manitoba & 46,249 & 59 & Tennessee, Knoxville & 40,000 \\
\hline 26 & California, Davis & 46,164 & 59 & Tulane & 40,000 \\
\hline 26 & California, Los Angeles & 46,164 & 59 & Utah & 40,000 \\
\hline 26 & California, San Diego & 46,164 & 59 & Washington U.-St. Louis & 40,000 \\
\hline 29 & McMaster & 45,945 & 66 & New Mexico & 39,372 \\
\hline 30 & Dartmouth & 45,500 & 67 & Rochester & 38,983 \\
\hline 31 & Hawaii & 45,000 & 68 & Texas Tech & 38,110 \\
\hline 31 & Nebraska & 45,000 & 69 & Oklahoma State & 38,000 \\
\hline 31 & North Carolina & 45,000 & 70 & Louisville & 37,000 \\
\hline 31 & Pittsburgh & 45,000 & 71 & Louisiana State & 36,000 \\
\hline 31 & SUNY Stony Brook & 45,000 & 72 & Case Western Reserve & 35,000 \\
\hline 31 & Tennessee, Memphis & 45,000 & 72 & South Carolina & 35,000 \\
\hline 31 & Virginia & 45,000 & & & \\
\hline
\end{tabular}

Beginning salary figures represent officially designated base, not necessarily salaries of actual incumbents. Canadian salaries are expressed in US dollars. 
Table 37: Median Professional Salaries in ARL University Medical Libraries

RANK ORDER TABLE, FY 2010-2011

\begin{tabular}{|c|c|c|c|c|c|}
\hline Rank & Institution & Salary & Rank & Institution & Salary \\
\hline 1 & Alberta & 105,587 & 38 & Howard & 60,776 \\
\hline 2 & Toronto & 89,289 & 39 & Pittsburgh & 60,227 \\
\hline 3 & Queen's & 85,492 & 40 & Utah & 60,173 \\
\hline 4 & British Columbia & 82,574 & 41 & Oklahoma & 60,019 \\
\hline 5 & California, Los Angeles & 81,876 & 42 & Ohio State & 59,919 \\
\hline 6 & McMaster & 76,034 & 43 & Washington & 59,040 \\
\hline 7 & Yale & 75,556 & 44 & Arizona & 58,651 \\
\hline 8 & Cornell & 75,542 & 45 & Temple & 57,680 \\
\hline 9 & Harvard & 75,531 & 46 & Michigan & 57,296 \\
\hline 10 & Connecticut & 75,152 & 47 & Nebraska & 57,200 \\
\hline 11 & Columbia & 73,803 & 48 & Emory & 56,959 \\
\hline 12 & Ottawa & 73,277 & 49 & Wisconsin & 56,669 \\
\hline 13 & California, San Diego & 72,345 & 50 & Louisville & 56,293 \\
\hline 14 & Manitoba & 71,754 & 51 & Illinois, Chicago & 55,697 \\
\hline 15 & SUNY Stony Brook & 71,317 & 52 & Duke & 55,164 \\
\hline 16 & Southern California & 71,300 & 53 & Kentucky & 54,282 \\
\hline 17 & Case Western Reserve & 71,055 & 54 & Rochester & 53,001 \\
\hline 18 & McGill & 70,071 & 55 & Iowa & 52,470 \\
\hline 19 & Saskatchewan & 69,604 & 56 & Florida State & 52,299 \\
\hline 20 & George Washington & 67,542 & 57 & Washington U.-St. Louis & 51,750 \\
\hline 21 & Calgary & 67,294 & 58 & Miami & 51,461 \\
\hline 22 & Vanderbilt & 67,060 & 59 & Tennessee, Memphis & 51,091 \\
\hline 23 & Johns Hopkins & 66,656 & 60 & Cincinnati & 50,688 \\
\hline 24 & Southern Illinois & 66,247 & 61 & Boston University & 50,142 \\
\hline 25 & Northwestern & 66,073 & 62 & Missouri & 50,017 \\
\hline 26 & SUNY Buffalo & 65,833 & 63 & Florida & 48,746 \\
\hline 27 & Virginia & 65,700 & 64 & Kansas & 47,370 \\
\hline 28 & Montreal & 65,596 & 65 & South Carolina & 46,692 \\
\hline 29 & North Carolina & 65,049 & 66 & Texas Tech & 45,634 \\
\hline 30 & California, Davis & 64,560 & 67 & Tulane & 38,684 \\
\hline 31 & Dartmouth & 64,415 & & Alabama & * \\
\hline 32 & Pennsylvania State & 62,982 & & Hawaii & * \\
\hline 33 & Minnesota & 62,846 & & Louisiana State & * \\
\hline 34 & New York University & 62,700 & & Oklahoma State & * \\
\hline 35 & New Mexico & 62,620 & & Tennessee, Knoxville & * \\
\hline 36 & Georgetown & 62,500 & & Wayne State & * \\
\hline 37 & Pennsylvania & 61,727 & & & \\
\hline
\end{tabular}

Salaries of directors are not included in the calculation of medians.

Alabama, Hawaii, Louisiana State, Oklahoma State, Tennessee-Knoxville, and Wayne State, are not ranked because they reported four or fewer individuals.

Canadian salaries are expressed in US dollars. 
Table 38: Average Professional Salaries in ARL University Medical Libraries

RANK ORDER TABLE, FY 2010-2011

\begin{tabular}{|c|c|c|c|c|c|}
\hline Rank & Institution & Salary & Rank & Institution & Salary \\
\hline 1 & Toronto & 88,333 & 38 & Dartmouth & 63,723 \\
\hline 2 & Alberta & 87,808 & 39 & Arizona & 63,668 \\
\hline 3 & Queen's & 86,981 & 40 & Southern Illinois & 63,036 \\
\hline 4 & California, Los Angeles & 82,699 & 41 & Emory & 62,851 \\
\hline 5 & British Columbia & 79,772 & 42 & Nebraska & 62,725 \\
\hline 6 & Ottawa & 78,426 & 43 & Pittsburgh & 62,379 \\
\hline 7 & Harvard & 78,066 & 44 & Ohio State & 62,170 \\
\hline 8 & Connecticut & 77,815 & 45 & Washington U.-St. Louis & 61,693 \\
\hline 9 & Yale & 76,505 & 46 & Cincinnati & 61,133 \\
\hline 10 & Cornell & 76,332 & 47 & Georgetown & 60,976 \\
\hline 11 & Calgary & 73,414 & 48 & Michigan & 60,267 \\
\hline 12 & Southern California & 72,947 & 49 & Oklahoma & 59,817 \\
\hline 13 & Manitoba & 72,939 & 50 & Illinois, Chicago & 59,275 \\
\hline 14 & McGill & 72,707 & 51 & Wisconsin & 58,988 \\
\hline 15 & Saskatchewan & 72,571 & 52 & Iowa & 58,790 \\
\hline 16 & California, Davis & 72,319 & 53 & Louisville & 58,207 \\
\hline 17 & Columbia & 70,545 & 54 & Duke & 58,074 \\
\hline 18 & SUNY Stony Brook & 70,087 & 55 & Temple & 57,880 \\
\hline 19 & California, San Diego & 69,359 & 56 & Rochester & 56,577 \\
\hline 20 & Montreal & 69,345 & 57 & Kentucky & 56,480 \\
\hline 21 & Johns Hopkins & 69,218 & 58 & Boston University & 55,599 \\
\hline 22 & North Carolina & 68,618 & 59 & Florida & 54,305 \\
\hline 23 & McMaster & 68,521 & 60 & Missouri & 53,831 \\
\hline 24 & SUNY Buffalo & 68,258 & 61 & Tennessee, Memphis & 53,636 \\
\hline 25 & Howard & 67,369 & 62 & Miami & 53,211 \\
\hline 26 & Case Western Reserve & 67,306 & 63 & Florida State & 51,041 \\
\hline 27 & New York University & 67,132 & 64 & Kansas & 50,383 \\
\hline 28 & George Washington & 66,900 & 65 & Texas Tech & 49,315 \\
\hline 29 & Utah & 66,845 & 66 & South Carolina & 49,291 \\
\hline 30 & Pennsylvania & 65,949 & 67 & Tulane & 40,606 \\
\hline 31 & New Mexico & 65,631 & & Alabama & * \\
\hline 32 & Northwestern & 65,507 & & Hawaii & * \\
\hline 33 & Virginia & 65,115 & & Louisiana State & * \\
\hline 34 & Pennsylvania State & 63,993 & & Oklahoma State & * \\
\hline 35 & Minnesota & 63,946 & & Tennessee, Knoxville & * \\
\hline 36 & Washington & 63,846 & & Wayne State & * \\
\hline 37 & Vanderbilt & 63,781 & & & \\
\hline
\end{tabular}

Salaries of directors are not included in the calculation of medians.

Alabama, Hawaii, Louisiana State, Oklahoma State, Tennessee-Knoxville, and Wayne State are not ranked because they reported four or fewer individuals.

Canadian salaries are expressed in US dollars. 
Table 39: Number and Average Salaries of ARL University Medical Librarians by Position AND SEx, FY 2010-2011

\begin{tabular}{|c|c|c|c|c|c|c|}
\hline \multirow[b]{2}{*}{ Position } & \multicolumn{2}{|c|}{ WOMEN } & \multicolumn{2}{|c|}{ MeN } & \multicolumn{2}{|c|}{ TOTAL } \\
\hline & Salary & No. & Salary & No. & Salary & No. \\
\hline Head, Medical & $\$ 126,693$ & 52 & $\$ 131,879$ & 16 & $\$ 127,913$ & 68 \\
\hline Associate Director & 84,544 & 37 & 96,530 & 15 & 88,002 & 52 \\
\hline Assistant Director & 68,005 & 35 & 75,635 & 7 & 69,276 & 42 \\
\hline Head, Branch & $\neq$ & 21 & $\neq$ & 1 & 68,371 & 22 \\
\hline Functional Specialist & 62,861 & 90 & 64,613 & 94 & 63,756 & 184 \\
\hline Subject Specialist & 63,429 & 62 & 59,891 & 11 & 62,896 & 73 \\
\hline \multicolumn{7}{|l|}{ Dept. Head: } \\
\hline Acquisitions & 65,136 & 16 & 63,049 & 6 & 64,567 & 22 \\
\hline Reference & 68,649 & 21 & 79,757 & 6 & 71,117 & 27 \\
\hline Cataloging & $\neq$ & 12 & $\neq$ & 2 & 69,295 & 14 \\
\hline Serials & $\neq$ & 6 & $\neq$ & 2 & 58,744 & 8 \\
\hline Documents/Maps & $\neq$ & 2 & . & & $\neq$ & 2 \\
\hline Circulation & 61,362 & 11 & 63,694 & 8 & 62,344 & 19 \\
\hline Rare Books/Manuscripts & $\neq$ & 2 & $\neq$ & 3 & 62,500 & 5 \\
\hline Computer Systems & 82,733 & 4 & 81,916 & 8 & 82,189 & 12 \\
\hline Other & 67,568 & 47 & 67,863 & 13 & 67,632 & 60 \\
\hline \multicolumn{7}{|l|}{ Reference: } \\
\hline Over 14 years experience & 69,080 & 93 & 70,633 & 24 & 69,398 & 117 \\
\hline 10 to 14 years experience & 63,374 & 29 & 69,088 & 8 & 64,609 & 37 \\
\hline 5 to 9 years experience & 60,213 & 38 & 53,954 & 8 & 59,125 & 46 \\
\hline Under 5 years experience & 50,747 & 35 & 49,087 & 7 & 50,470 & 42 \\
\hline \multicolumn{7}{|l|}{ Cataloging: } \\
\hline Over 14 years experience & $\neq$ & 4 & $\neq$ & 2 & 67,224 & 6 \\
\hline 10 to 14 years experience & $\neq$ & 3 & . & & $\neq$ & 3 \\
\hline 5 to 9 years experience & 48,427 & 4 & . & & 48,427 & 4 \\
\hline Under 5 years experience & 51,050 & 5 & . & & 51,050 & 5 \\
\hline \multicolumn{7}{|l|}{ Other: } \\
\hline Over 14 years experience & 64,245 & 30 & 64,291 & 10 & 64,256 & 40 \\
\hline 10 to 14 years experience & $\neq$ & 12 & $\neq$ & 2 & 56,317 & 14 \\
\hline 5 to 9 years experience & 55,550 & 18 & 57,587 & 6 & 56,059 & 24 \\
\hline Under 5 years experience & 46,035 & 18 & 54,009 & 8 & 48,489 & 26 \\
\hline All Positions & $\$ 69,092$ & 707 & $\$ 70,912$ & 267 & $\$ 69,591$ & 974 \\
\hline
\end{tabular}

Canadian salaries are expressed in US dollars.

₹ Salary data are not published when fewer than four individuals are involved in either category.

. No positions reported in this category. 
Table 40: Number and Average Years of Experience of ARL University Medical Librarians by Position And Sex, FY 2010-2011

\begin{tabular}{|c|c|c|c|c|c|c|}
\hline \multirow[b]{2}{*}{ Position } & \multicolumn{2}{|c|}{ WOMEN } & \multicolumn{2}{|c|}{ MeN } & \multicolumn{2}{|c|}{ Total } \\
\hline & Years & No. & Years & No. & Years & No. \\
\hline Head, Medical & 30.3 & 52 & 29.5 & 16 & 30.1 & 68 \\
\hline Associate Director & 22.6 & 37 & 26.1 & 15 & 23.6 & 52 \\
\hline Assistant Director & 20.8 & 35 & 16.4 & 7 & 20.1 & 42 \\
\hline Head, Branch & 19.6 & 21 & 8.0 & 1 & 19.1 & 22 \\
\hline Functional Specialist & 12.7 & 90 & 10.1 & 94 & 11.3 & 184 \\
\hline Subject Specialist & 16.6 & 62 & 12.5 & 11 & 16.0 & 73 \\
\hline \multicolumn{7}{|l|}{ Dept. Head: } \\
\hline Acquisitions & 18.6 & 16 & 15.2 & 6 & 17.6 & 22 \\
\hline Reference & 20.8 & 21 & 23.2 & 6 & 21.3 & 27 \\
\hline Cataloging & 20.3 & 12 & 22.0 & 2 & 20.6 & 14 \\
\hline Serials & 17.0 & 6 & 15.5 & 2 & 16.6 & 8 \\
\hline Documents/Maps & 10.5 & 2 & . & & 10.5 & 2 \\
\hline Circulation & 20.1 & 11 & 9.6 & 8 & 15.7 & 19 \\
\hline Rare Books/Manuscripts & 21.0 & 2 & 25.3 & 3 & 23.6 & 5 \\
\hline Computer Systems & 18.8 & 4 & 18.8 & 8 & 18.8 & 12 \\
\hline Other & 17.1 & 47 & 14.9 & 13 & 16.7 & 60 \\
\hline Public services & 13.6 & 53 & 9.3 & 15 & 12.6 & 68 \\
\hline Technical services & 13.2 & 13 & 18.8 & 6 & 15.0 & 19 \\
\hline Administration & 14.3 & 12 & 15.2 & 5 & 14.6 & 17 \\
\hline Reference & 15.7 & 195 & 15.4 & 47 & 15.6 & 242 \\
\hline Cataloger & 9.8 & 16 & 27.0 & 2 & 11.7 & 18 \\
\hline All Positions & 17.3 & 707 & 14.9 & 267 & 16.7 & 974 \\
\hline
\end{tabular}

. No positions were reported in this category. 
Table 41: Number and Average Salaries of ARL University Medical Librarians by YeARs of EXPERIENCE AND SEX, FY 2010-2011

\begin{tabular}{|c|c|c|c|c|c|c|c|}
\hline \multirow[b]{2}{*}{ Experience } & \multicolumn{2}{|c|}{ WOMEN } & \multicolumn{2}{|c|}{ MeN } & \multicolumn{2}{|c|}{ Total } & \multirow{2}{*}{$\begin{array}{r}\% \text { of } \\
\text { TotaL }\end{array}$} \\
\hline & Salary & No. & Salary & No. & Salary & No. & \\
\hline $0-3$ years & $\$ 55,288$ & 85 & $\$ 57,331$ & 53 & $\$ 56,073$ & 138 & $14 \%$ \\
\hline $4-7$ years & 55,633 & 102 & 56,790 & 34 & 55,922 & 136 & $14 \%$ \\
\hline 8-11 years & 61,589 & 94 & 69,070 & 41 & 63,861 & 135 & $14 \%$ \\
\hline $12-15$ years & 68,313 & 68 & 69,408 & 24 & 68,599 & 92 & $9 \%$ \\
\hline 16-19 years & 68,195 & 68 & 73,987 & 28 & 69,884 & 96 & $10 \%$ \\
\hline 20-23 years & 70,567 & 51 & 80,979 & 17 & 73,170 & 68 & $7 \%$ \\
\hline 24-27 years & 74,598 & 63 & 90,468 & 17 & 77,970 & 80 & $8 \%$ \\
\hline 28-31 years & 79,810 & 71 & 78,316 & 22 & 79,457 & 93 & $10 \%$ \\
\hline $32-35$ years & 86,855 & 56 & 79,489 & 21 & 84,846 & 77 & $8 \%$ \\
\hline over 35 years & 93,326 & 49 & 108,785 & 10 & 95,946 & 59 & $6 \%$ \\
\hline All Positions & $\$ 69,092$ & 707 & $\$ 70,912$ & 267 & $\$ 69,591$ & 974 & $100 \%$ \\
\hline
\end{tabular}

Canadian salaries are expressed in US dollars. 

ARL UNIVERSITY LAW LIBRARIES

TABLES 42-48 
Table 42: Filled Positions; Average, Median, and Beginning Salaries; and Average Years of EXPERIENCE IN ARL UNIVERSITY LAW LibRARIES, FY 2010-2011

\begin{tabular}{|c|c|c|c|c|c|}
\hline Institution & $\begin{array}{c}\text { Filled } \\
\text { Positions }\end{array}$ & $\begin{array}{c}\text { Average } \\
\text { Salary }\end{array}$ & $\begin{array}{l}\text { Median } \\
\text { Salary }\end{array}$ & $\begin{array}{l}\text { Beginning } \\
\text { Salary }\end{array}$ & $\begin{array}{c}\text { Average Yrs. } \\
\text { Exp. }\end{array}$ \\
\hline Alberta & 4 & $\neq$ & $\neq$ & $\$ 52,833$ & 29.5 \\
\hline Arizona & 8 & $\$ 64,267$ & $\$ 65,341$ & 50,000 & 18.3 \\
\hline Arizona State & 6 & 68,202 & 73,168 & 46,500 & 25.7 \\
\hline Boston University & 11 & 74,520 & 69,700 & 55,000 & 17.8 \\
\hline Boston College & 16 & 68,694 & 67,000 & 43,350 & 18.2 \\
\hline British Columbia & 3 & $\neq$ & $\neq$ & 52,420 & 23.7 \\
\hline Calgary & 3 & $\neq$ & $\neq$ & 54,945 & 19.0 \\
\hline California, Davis & 8 & 74,131 & 68,892 & 46,164 & 18.9 \\
\hline California, Irvine & 6 & 85,995 & 82,524 & 46,144 & 11.5 \\
\hline California, Los Angeles & 16 & 77,676 & 82,524 & 46,164 & 14.6 \\
\hline Case Western Reserve & 12 & 69,200 & 67,467 & 35,000 & 20.3 \\
\hline Cincinnati & 8 & 64,512 & 54,384 & 50,000 & 19.5 \\
\hline Colorado & 8 & 72,314 & 59,901 & 45,000 & 19.0 \\
\hline Columbia & 17 & 78,515 & 72,891 & 52,000 & 14.0 \\
\hline Connecticut & 9 & 72,135 & 72,097 & 50,000 & 21.8 \\
\hline Cornell & 8 & 78,587 & 72,828 & 59,200 & 14.6 \\
\hline Duke & 11 & 69,175 & 63,750 & 60,000 & 16.9 \\
\hline Emory & 9 & 59,559 & 56,842 & 42,000 & 12.6 \\
\hline Florida & 8 & 58,379 & 53,162 & 50,000 & 11.1 \\
\hline Florida State & 9 & 54,208 & 54,815 & 50,000 & 19.6 \\
\hline George Washington & 21 & 89,173 & 82,890 & 58,000 & 15.3 \\
\hline Georgetown & 25 & 77,644 & 71,710 & 54,000 & 12.4 \\
\hline Georgia & 8 & 56,405 & 55,752 & 50,000 & 14.0 \\
\hline Harvard & 37 & 81,584 & 77,988 & 53,093 & 14.5 \\
\hline Hawaii & 5 & 84,551 & 81,193 & 45,000 & 15.6 \\
\hline Houston & 12 & 57,094 & 52,788 & 51,000 & 16.3 \\
\hline Howard & 6 & 48,423 & 49,914 & 51,000 & 21.5 \\
\hline Illinois, Urbana & 9 & 64,664 & 67,246 & 53,500 & 16.8 \\
\hline Indiana & 10 & 69,325 & 64,923 & 40,400 & 22.0 \\
\hline Iowa & 17 & 73,788 & 73,250 & 41,000 & 19.4 \\
\hline Kansas & 7 & 47,022 & 44,926 & 42,000 & 9.3 \\
\hline Kentucky & 5 & 57,520 & 52,000 & 50,000 & 13.8 \\
\hline Louisiana State & 9 & 57,380 & 55,846 & 50,000 & 18.2 \\
\hline Louisville & 6 & 60,053 & 59,931 & 37,000 & 20.3 \\
\hline McGill & 5 & 83,096 & 76,437 & 47,366 & 19.6 \\
\hline Manitoba & 3 & $\neq$ & $\neq$ & 46,249 & 29.3 \\
\hline Miami & 13 & 60,319 & 57,688 & 45,000 & 15.7 \\
\hline Michigan & 11 & 83,231 & 81,014 & 49,000 & 15.4 \\
\hline Minnesota & 14 & 75,686 & 68,200 & 46,000 & 20.4 \\
\hline Missouri & 8 & 55,073 & 57,327 & 40,000 & 14.6 \\
\hline Montreal & 4 & $\neq$ & $\neq$ & 50,297 & 8.0 \\
\hline Nebraska & 5 & 63,975 & 63,772 & 45,000 & 18.4 \\
\hline
\end{tabular}

78 · ARL Annual Salary Survey 2010-2011 
Table 42: Filled Positions; Average, Median, and Beginning Salaries; and Average Years of EXPERIENCE IN ARL UNIVERSITY LAW LibRARIES, FY 2010-2011

\begin{tabular}{|c|c|c|c|c|c|}
\hline Institution & $\begin{array}{c}\text { Filled } \\
\text { Positions }\end{array}$ & $\begin{array}{c}\text { Average } \\
\text { Salary }\end{array}$ & $\begin{array}{c}\text { Median } \\
\text { Salary }\end{array}$ & $\begin{array}{l}\text { Beginning } \\
\text { Salary }\end{array}$ & $\begin{array}{c}\text { Average Yrs. } \\
\text { Exp. }\end{array}$ \\
\hline New Mexico & 8 & 64,782 & 59,760 & 50,000 & 12.8 \\
\hline New York University & 19 & 80,014 & 76,203 & 60,000 & 22.6 \\
\hline North Carolina & 12 & 74,737 & 68,000 & 55,000 & 16.1 \\
\hline Northwestern & 10 & 65,860 & 61,503 & 44,000 & 22.3 \\
\hline Notre Dame & 14 & 70,802 & 66,500 & 44,000 & 18.1 \\
\hline Ohio State & 6 & 68,061 & 61,510 & 46,000 & 12.5 \\
\hline Oklahoma & 7 & 57,410 & 51,269 & 45,000 & 15.7 \\
\hline Oregon & 6 & 53,544 & 57,846 & 42,000 & 17.5 \\
\hline Ottawa & 4 & $\neq$ & $\neq$ & 47,538 & 18.8 \\
\hline Pennsylvania & 12 & 67,497 & 65,462 & 49,000 & 13.3 \\
\hline Pennsylvania State & 9 & 79,637 & 73,992 & 57,000 & 23.0 \\
\hline Queen's & 3 & $\neq$ & $\neq$ & 50,446 & 10.7 \\
\hline Rutgers, Camden & 8 & 75,469 & 87,500 & 62,000 & 22.8 \\
\hline Rutgers, Newark & 10 & 73,957 & 65,760 & 62,000 & 17.5 \\
\hline Saskatchewan & 3 & $\neq$ & $\neq$ & 52,202 & 23.0 \\
\hline South Carolina & 11 & 65,437 & 68,375 & 50,000 & 12.7 \\
\hline Southern Illinois & 4 & $\neq$ & $\neq$ & 50,000 & 7.0 \\
\hline SUNY Buffalo & 11 & 74,077 & 74,004 & 55,000 & 14.0 \\
\hline Syracuse & 10 & 57,621 & 54,630 & 46,700 & 14.5 \\
\hline Temple & 10 & 63,848 & 53,203 & 44,004 & 24.1 \\
\hline Tennessee & 7 & 65,954 & 62,718 & 48,000 & 15.7 \\
\hline Texas & 15 & 59,425 & 53,429 & 40,000 & 16.1 \\
\hline Texas Tech & 8 & 60,612 & 56,990 & 47,000 & 12.8 \\
\hline Toronto & 6 & 83,988 & 81,765 & 49,451 & 14.8 \\
\hline Tulane & 6 & 61,297 & 59,455 & 40,000 & 19.3 \\
\hline Utah & 8 & 57,095 & 52,530 & 41,500 & 17.5 \\
\hline Vanderbilt & 5 & 70,083 & 61,524 & 41,000 & 21.0 \\
\hline Virginia & 14 & 68,562 & 63,500 & 63,500 & 16.1 \\
\hline Washington & 16 & 72,458 & 65,735 & 60,000 & 22.8 \\
\hline Washington U.-St. Louis & 10 & 63,709 & 58,560 & 48,000 & 19.9 \\
\hline Wayne State & 4 & $\neq$ & $\neq$ & 45,000 & 24.0 \\
\hline Western Ontario & 3 & $\neq$ & $\neq$ & 47,836 & 15.3 \\
\hline Wisconsin & 12 & 64,416 & 58,954 & 40,526 & 24.1 \\
\hline Yale & 18 & 85,403 & 85,911 & 50,500 & 20.1 \\
\hline York & 5 & 87,978 & 90,448 & 49,000 & 16.2 \\
\hline
\end{tabular}

Directors are included in figures for filled positions and average years of experience, but not in either the average or median salary statistics. Canadian salaries are expressed in US dollars.

‡ Salary data are not published when fewer than four individuals are involved. 
Table 43: Beginning Professional Salaries in ARL University LaW Libraries

Rank Order Table, FY 2010-2011

\begin{tabular}{|c|c|c|c|c|c|}
\hline Rank & Institution & Salary & Rank & Institution & Salary \\
\hline 1 & Virginia & 63,500 & 38 & York & 49,000 \\
\hline 2 & Rutgers, Camden & 62,000 & 41 & Tennessee & 48,000 \\
\hline 2 & Rutgers, Newark & 62,000 & 41 & Washington U.-St. Louis & 48,000 \\
\hline 4 & Duke & 60,000 & 43 & Western Ontario & 47,836 \\
\hline 4 & New York University & 60,000 & 44 & Ottawa & 47,538 \\
\hline 4 & Washington & 60,000 & 45 & McGill & 47,366 \\
\hline 7 & Cornell & 59,200 & 46 & Texas Tech & 47,000 \\
\hline 8 & George Washington & 58,000 & 47 & Syracuse & 46,700 \\
\hline 9 & Pennsylvania State & 57,000 & 48 & Arizona State & 46,500 \\
\hline 10 & Boston University & 55,000 & 49 & Manitoba & 46,249 \\
\hline 10 & North Carolina & 55,000 & 50 & California, Davis & 46,164 \\
\hline 10 & SUNY Buffalo & 55,000 & 50 & California, Los Angeles & 46,164 \\
\hline 13 & Calgary & 54,945 & 52 & California, Irvine & 46,144 \\
\hline 14 & Georgetown & 54,000 & 53 & Minnesota & 46,000 \\
\hline 15 & Illinois, Urbana & 53,500 & 53 & Ohio State & 46,000 \\
\hline 16 & Harvard & 53,093 & 55 & Colorado & 45,000 \\
\hline 17 & Alberta & 52,833 & 55 & Hawaii & 45,000 \\
\hline 18 & British Columbia & 52,420 & 55 & Miami & 45,000 \\
\hline 19 & Saskatchewan & 52,202 & 55 & Nebraska & 45,000 \\
\hline 20 & Columbia & 52,000 & 55 & Oklahoma & 45,000 \\
\hline 21 & Houston & 51,000 & 55 & Wayne State & 45,000 \\
\hline 21 & Howard & 51,000 & 61 & Temple & 44,004 \\
\hline 23 & Yale & 50,500 & 62 & Northwestern & 44,000 \\
\hline 24 & Queen's & 50,446 & 62 & Notre Dame & 44,000 \\
\hline 25 & Montreal & 50,297 & 64 & Boston College & 43,350 \\
\hline 26 & Arizona & 50,000 & 65 & Emory & 42,000 \\
\hline 26 & Cincinnati & 50,000 & 65 & Kansas & 42,000 \\
\hline 26 & Connecticut & 50,000 & 65 & Oregon & 42,000 \\
\hline 26 & Florida & 50,000 & 68 & Utah & 41,500 \\
\hline 26 & Florida State & 50,000 & 69 & Iowa & 41,000 \\
\hline 26 & Georgia & 50,000 & 69 & Vanderbilt & 41,000 \\
\hline 26 & Kentucky & 50,000 & 71 & Wisconsin & 40,526 \\
\hline 26 & Louisiana State & 50,000 & 72 & Indiana & 40,400 \\
\hline 26 & New Mexico & 50,000 & 73 & Missouri & 40,000 \\
\hline 26 & South Carolina & 50,000 & 73 & Texas & 40,000 \\
\hline 26 & Southern Illinois & 50,000 & 73 & Tulane & 40,000 \\
\hline 37 & Toronto & 49,451 & 76 & Louisville & 37,000 \\
\hline 38 & Michigan & 49,000 & 77 & Case Western Reserve & 35,000 \\
\hline 38 & Pennsylvania & 49,000 & & & \\
\hline
\end{tabular}

Beginning salary figures represent officially designated base, not necessarily salaries of actual incumbents.

Canadian salaries are expressed in US dollars. 
Table 44: Median Professional Salaries in ARL University Law Libraries

Rank Order Table, FY 2010-2011

\begin{tabular}{|c|c|c|c|c|c|}
\hline Rank & Institution & Salary & Rank & Institution & Salary \\
\hline 1 & York & 90,448 & 40 & Ohio State & 61,510 \\
\hline 2 & Rutgers, Camden & 87,500 & 41 & Northwestern & 61,503 \\
\hline 3 & Yale & 85,911 & 42 & Louisville & 59,931 \\
\hline 4 & George Washington & 82,890 & 43 & Colorado & 59,901 \\
\hline 5 & California, Irvine & 82,524 & 44 & New Mexico & 59,760 \\
\hline 5 & California, Los Angeles & 82,524 & 45 & Tulane & 59,455 \\
\hline 7 & Toronto & 81,765 & 46 & Wisconsin & 58,954 \\
\hline 8 & Hawaii & 81,193 & 47 & Washington U.-St. Louis & 58,560 \\
\hline 9 & Michigan & 81,014 & 48 & Oregon & 57,846 \\
\hline 10 & Harvard & 77,988 & 49 & Miami & 57,688 \\
\hline 11 & McGill & 76,437 & 50 & Missouri & 57,327 \\
\hline 12 & New York University & 76,203 & 51 & Texas Tech & 56,990 \\
\hline 13 & SUNY Buffalo & 74,004 & 52 & Emory & 56,842 \\
\hline 14 & Pennsylvania State & 73,992 & 53 & Louisiana State & 55,846 \\
\hline 15 & Iowa & 73,250 & 54 & Georgia & 55,752 \\
\hline 16 & Arizona State & 73,168 & 55 & Florida State & 54,815 \\
\hline 17 & Columbia & 72,891 & 56 & Syracuse & 54,630 \\
\hline 18 & Cornell & 72,828 & 57 & Cincinnati & 54,384 \\
\hline 19 & Connecticut & 72,097 & 58 & Texas & 53,429 \\
\hline 20 & Georgetown & 71,710 & 59 & Temple & 53,203 \\
\hline 21 & Boston University & 69,700 & 60 & Florida & 53,162 \\
\hline 22 & California, Davis & 68,892 & 61 & Houston & 52,788 \\
\hline 23 & South Carolina & 68,375 & 62 & Utah & 52,530 \\
\hline 24 & Minnesota & 68,200 & 63 & Kentucky & 52,000 \\
\hline 25 & North Carolina & 68,000 & 64 & Oklahoma & 51,269 \\
\hline 26 & Case Western Reserve & 67,467 & 65 & Howard & 49,914 \\
\hline 27 & Illinois, Urbana & 67,246 & 66 & Kansas & 44,926 \\
\hline 28 & Boston College & 67,000 & & Alberta & * \\
\hline 29 & Notre Dame & 66,500 & & British Columbia & * \\
\hline 30 & Rutgers, Newark & 65,760 & & Calgary & * \\
\hline 31 & Washington & 65,735 & & Manitoba & * \\
\hline 32 & Pennsylvania & 65,462 & & Montreal & * \\
\hline 33 & Arizona & 65,341 & & Ottawa & * \\
\hline 34 & Indiana & 64,923 & & Queen's & * \\
\hline 35 & Nebraska & 63,772 & & Saskatchewan & * \\
\hline 36 & Duke & 63,750 & & Southern Illinois & * \\
\hline 37 & Virginia & 63,500 & & Wayne State & * \\
\hline 38 & Tennessee & 62,718 & & Western Ontario & * \\
\hline 39 & Vanderbilt & 61,524 & & & \\
\hline
\end{tabular}

Salaries of directors are not included in the calculation of medians.

Alberta, British Columbia, Calgary, Manitoba, Montreal, Ottawa, Queen's, Saskatchewan, Southern Illinois, Wayne State, and Western Ontario are not ranked because they reported four or fewer individuals.

Canadian salaries are expressed in US dollars. 
Table 45: Average Professional Salaries in ARL University Law Libraries

Rank Order Table, FY 2010-2011

\begin{tabular}{|c|c|c|c|c|c|}
\hline Rank & Institution & Salary & Rank & Institution & Salary \\
\hline 1 & George Washington & 89,173 & 40 & New Mexico & 64,782 \\
\hline 2 & York & 87,978 & 41 & Illinois, Urbana & 64,664 \\
\hline 3 & California, Irvine & 85,995 & 42 & Cincinnati & 64,512 \\
\hline 4 & Yale & 85,403 & 43 & Wisconsin & 64,416 \\
\hline 5 & Hawaii & 84,551 & 44 & Arizona & 64,267 \\
\hline 6 & Toronto & 83,988 & 45 & Nebraska & 63,975 \\
\hline 7 & Michigan & 83,231 & 46 & Temple & 63,848 \\
\hline 8 & McGill & 83,096 & 47 & Washington U.-St. Louis & 63,709 \\
\hline 9 & Harvard & 81,584 & 48 & Tulane & 61,297 \\
\hline 10 & New York University & 80,014 & 49 & Texas Tech & 60,612 \\
\hline 11 & Pennsylvania State & 79,637 & 50 & Miami & 60,319 \\
\hline 12 & Cornell & 78,587 & 51 & Louisville & 60,053 \\
\hline 13 & Columbia & 78,515 & 52 & Emory & 59,559 \\
\hline 14 & California, Los Angeles & 77,676 & 53 & Texas & 59,425 \\
\hline 15 & Georgetown & 77,644 & 54 & Florida & 58,379 \\
\hline 16 & Minnesota & 75,686 & 55 & Syracuse & 57,621 \\
\hline 17 & Rutgers, Camden & 75,469 & 56 & Kentucky & 57,520 \\
\hline 18 & North Carolina & 74,737 & 57 & Oklahoma & 57,410 \\
\hline 19 & Boston University & 74,520 & 58 & Louisiana State & 57,380 \\
\hline 20 & California, Davis & 74,131 & 59 & Utah & 57,095 \\
\hline 21 & SUNY Buffalo & 74,077 & 60 & Houston & 57,094 \\
\hline 22 & Rutgers, Newark & 73,957 & 61 & Georgia & 56,405 \\
\hline 23 & Iowa & 73,788 & 62 & Missouri & 55,073 \\
\hline 24 & Washington & 72,458 & 63 & Florida State & 54,208 \\
\hline 25 & Colorado & 72,314 & 64 & Oregon & 53,544 \\
\hline 26 & Connecticut & 72,135 & 65 & Howard & 48,423 \\
\hline 27 & Notre Dame & 70,802 & 66 & Kansas & 47,022 \\
\hline 28 & Vanderbilt & 70,083 & & Alberta & * \\
\hline 29 & Indiana & 69,325 & & British Columbia & * \\
\hline 30 & Case Western Reserve & 69,200 & & Calgary & * \\
\hline 31 & Duke & 69,175 & & Manitoba & * \\
\hline 32 & Boston College & 68,694 & & Montreal & * \\
\hline 33 & Virginia & 68,562 & & Ottawa & * \\
\hline 34 & Arizona State & 68,202 & & Queen's & * \\
\hline 35 & Ohio State & 68,061 & & Saskatchewan & * \\
\hline 36 & Pennsylvania & 67,497 & & Southern Illinois & * \\
\hline 37 & Tennessee & 65,954 & & Wayne State & * \\
\hline 38 & Northwestern & 65,860 & & Western Ontario & * \\
\hline 39 & South Carolina & 65,437 & & & \\
\hline
\end{tabular}

Salaries of directors are not included in the calculation of medians.

Alberta, British Columbia, Calgary, Manitoba, Montreal, Ottawa, Queen's, Saskatchewan, Southern Illinois, Wayne State, and Western Ontario are not ranked because they reported four or fewer individuals.

Canadian salaries are expressed in US dollars. 
Table 46: Number and Average Salaries of ARL University LaW Librarians by Position AND Sex, FY 2010-2011

\begin{tabular}{|c|c|c|c|c|c|c|}
\hline \multirow[b]{2}{*}{ Position } & \multicolumn{2}{|c|}{ WOMEN } & \multicolumn{2}{|c|}{ Men } & \multicolumn{2}{|c|}{ Total } \\
\hline & Salary & No. & Salary & No. & Salary & No. \\
\hline Head, Law & $\$ 156,041$ & 37 & $\$ 150,636$ & 35 & $\$ 153,414$ & 72 \\
\hline Associate Director & 96,343 & 35 & 98,129 & 21 & 97,013 & 56 \\
\hline Assistant Director & 88,203 & 29 & 88,835 & 12 & 88,388 & 41 \\
\hline Functional Specialist & 61,267 & 26 & 66,671 & 28 & 64,069 & 54 \\
\hline Subject Specialist & 73,005 & 30 & 81,425 & 10 & 75,110 & 40 \\
\hline \multicolumn{7}{|l|}{ Dept. Head: } \\
\hline Acquisitions & 66,681 & 27 & 59,784 & 8 & 65,105 & 35 \\
\hline Reference & 77,529 & 18 & 78,105 & 6 & 77,673 & 24 \\
\hline Cataloging & 70,736 & 26 & 65,520 & 4 & 70,040 & 30 \\
\hline Serials & $\neq$ & 5 & $\neq$ & 1 & 69,298 & 6 \\
\hline Documents/Maps & $\neq$ & 7 & $\neq$ & 1 & 67,239 & 8 \\
\hline Circulation & 64,483 & 17 & 57,528 & 6 & 62,668 & 23 \\
\hline Rare Books/Manuscripts & $\neq$ & 3 & . & & $\neq$ & 3 \\
\hline Computer Systems & $\neq$ & 2 & $\neq$ & 6 & 83,753 & 8 \\
\hline Other & 75,660 & 23 & 73,210 & 9 & 74,971 & 32 \\
\hline \multicolumn{7}{|l|}{ Reference: } \\
\hline Over 14 years experience & 74,618 & 47 & 74,788 & 21 & 74,671 & 68 \\
\hline 10 to 14 years experience & 57,743 & 11 & 61,892 & 10 & 59,719 & 21 \\
\hline 5 to 9 years experience & 63,694 & 37 & 64,895 & 21 & 64,129 & 58 \\
\hline Under 5 years experience & 59,321 & 43 & 56,980 & 26 & 58,439 & 69 \\
\hline \multicolumn{7}{|l|}{ Cataloging } \\
\hline Over 14 years experience & 63,562 & 20 & 72,179 & 5 & 65,285 & 25 \\
\hline 10 to 14 years experience & $\neq$ & 4 & $\neq$ & 1 & 57,455 & 5 \\
\hline 5 to 9 years experience & 56,873 & 7 & . & & 56,873 & 7 \\
\hline Under 5 years experience & $\neq$ & 2 & $\neq$ & 3 & 60,498 & 5 \\
\hline \multicolumn{7}{|l|}{ Other: } \\
\hline Over 14 years experience & 70,234 & 12 & 65,599 & 5 & 68,871 & 17 \\
\hline 10 to 14 years experience & $\neq$ & 3 & $\neq$ & 1 & $\neq$ & 4 \\
\hline 5 to 9 years experience & 49,333 & 4 & 72,482 & 4 & 60,907 & 8 \\
\hline Under 5 years experience & $\neq$ & 13 & $\neq$ & 2 & 54,461 & 15 \\
\hline All Positions & $\$ 77,036$ & 488 & $\$ 82,751$ & 246 & $\$ 78,951$ & 734 \\
\hline
\end{tabular}

Canadian salaries are expressed in US dollars.

‡ Salary data are not published when fewer than four individuals are involved in either category.

. No positions were reported in this category. 
Table 47: Number and Average Years of Experience of ARL University LaW Librarians by Position And Sex, FY 2010-2011

\begin{tabular}{|c|c|c|c|c|c|c|}
\hline \multirow[b]{2}{*}{ Position } & \multicolumn{2}{|c|}{ WOMEN } & \multicolumn{2}{|c|}{ MeN } & \multicolumn{2}{|c|}{ Total } \\
\hline & Years & No. & Years & No. & Years & No. \\
\hline Head, Law & 29.5 & 37 & 23.1 & 35 & 26.3 & 72 \\
\hline Associate Director & 24.8 & 35 & 19.5 & 21 & 22.8 & 56 \\
\hline Assistant Director & 20.4 & 29 & 24.5 & 12 & 21.6 & 41 \\
\hline Functional Specialist & 12.7 & 26 & 10.5 & 28 & 11.5 & 54 \\
\hline Subject Specialist & 17.8 & 30 & 21.8 & 10 & 18.8 & 40 \\
\hline \multicolumn{7}{|l|}{ Dept. Head: } \\
\hline Acquisitions & 23.0 & 27 & 13.5 & 8 & 20.8 & 35 \\
\hline Reference & 16.9 & 18 & 21.5 & 6 & 18.0 & 24 \\
\hline Cataloging & 26.2 & 26 & 22.5 & 4 & 25.7 & 30 \\
\hline Serials & 18.8 & 5 & 8.0 & 1 & 17.0 & 6 \\
\hline Documents/Maps & 28.4 & 7 & 13.0 & 1 & 26.5 & 8 \\
\hline Circulation & 17.2 & 17 & 12.2 & 6 & 15.9 & 23 \\
\hline Rare Books / Manuscripts & 17.0 & 3 & & & 17.0 & 3 \\
\hline Computer Systems & 22.5 & 2 & 19.8 & 6 & 20.5 & 8 \\
\hline Other & 18.0 & 23 & 15.8 & 9 & 17.4 & 32 \\
\hline Public services & 12.1 & 11 & 13.1 & 7 & 12.5 & 18 \\
\hline Technical services & 11.5 & 13 & 14.3 & 4 & 12.2 & 17 \\
\hline Administration & 15.6 & 8 & 25.0 & 1 & 16.7 & 9 \\
\hline Reference & 12.1 & 138 & 10.6 & 78 & 11.6 & 216 \\
\hline Cataloger & 19.8 & 33 & 17.7 & 9 & 19.3 & 42 \\
\hline All Positions & 18.1 & 488 & 15.7 & 246 & 17.3 & 734 \\
\hline
\end{tabular}

. No positions were reported in this category. 
Table 48: Number and Average Salaries of ARL University Law Librarians by YeARs of EXPERIENCE AND SEX, FY 2010-2011

\begin{tabular}{|c|c|c|c|c|c|c|c|}
\hline \multirow[b]{2}{*}{ Experience } & \multicolumn{2}{|c|}{ WOMEN } & \multicolumn{2}{|c|}{ MeN } & \multicolumn{2}{|c|}{ Total } & \multirow{2}{*}{$\begin{array}{r}\% \text { of } \\
\text { TotaL }\end{array}$} \\
\hline & Salary & No. & Salary & No. & Salary & No. & \\
\hline $0-3$ years & $\$ 57,382$ & 62 & $\$ 59,278$ & 34 & $\$ 58,054$ & 96 & $13 \%$ \\
\hline $4-7$ years & 62,747 & 70 & 64,114 & 41 & 63,252 & 111 & $15 \%$ \\
\hline 8-11 years & 69,850 & 39 & 69,083 & 28 & 69,530 & 67 & $9 \%$ \\
\hline $12-15$ years & 70,354 & 58 & 85,318 & 28 & 75,226 & 86 & $12 \%$ \\
\hline 16-19 years & 71,967 & 49 & 87,101 & 23 & 76,801 & 72 & $10 \%$ \\
\hline 20-23 years & 89,316 & 38 & 91,057 & 25 & 90,007 & 63 & $9 \%$ \\
\hline 24-27 years & 85,832 & 35 & 89,627 & 23 & 87,337 & 58 & $8 \%$ \\
\hline 28-31 years & 87,335 & 49 & 117,575 & 21 & 96,407 & 70 & $10 \%$ \\
\hline 32-35 years & 95,806 & 43 & 113,406 & 21 & 101,581 & 64 & $9 \%$ \\
\hline over 35 years & $\neq$ & 45 & $\neq$ & 2 & 100,273 & 47 & $6 \%$ \\
\hline All Positions & $\$ 77,036$ & 488 & $\$ 82,751$ & 246 & $\$ 78,951$ & 734 & $100 \%$ \\
\hline
\end{tabular}

Canadian salaries are expressed in US dollars.

‡ Salary data are not published when fewer than four individuals are involved in either category. 

UNIVERSITY LiBRARY QUESTIONNAIRE AND INSTRUCTIONS 


\author{
ARL AnNual Salary Survey 2010-11 \\ University Library Questionnaire \\ GENERAL AND DATA INPUT (EXCEL) INSTRUCTIONS
}

http://www.arl.org/stats/annualsurveys/salary/

\title{
GENERAL OVERVIEW
}

- Use the newly available Web form for your data submission:

- University Libraries: http://www.formstack.com/forms/?987723-c6BynijupA

Fill in Part I on the Web and upload your file for Part II through the same interface.

NOTE: You must complete the entire submission in a single session. The Web interface does

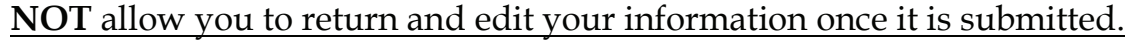

- This survey is concerned with professional positions only. Since the criteria for determining professional status vary among libraries, there is no attempt to define the term "professional." Each library should report the salaries of those staff members it considers professionals, irrespective of faculty status or membership in a collective bargaining unit, including, when appropriate, staff who are not librarians in the strict sense of the term, such as computer experts, systems analysts, budget officers, etc.

- Report individual salaries for the Main, Law, and Medical library on the separate template using Microsoft Excel (see http://www.arl.org/stats/annualsurveys/salary/salform10.shtml). A generic template is available. Add your institution's ARL Library Institution Code [LibID]. (See http://www.arl.org/stats/annualsurveys/surveycoord/instno_inam.shtml if you do not know your code.)

- Use "Percent" to determine if an employee works full-time or part-time. All full-time employees have Percent $=1.00$, i.e., they work $100 \%$ of a full-time schedule. If Percent is less than 1.00, then the employee works that fraction of a full-time schedule. For example, a $65 \%$ time appointment would be entered as 0.65 . Calculate the percent appointment by dividing the amount of time an employee works by the amount considered to be the norm for fulltime employment at your institution. For example, if a full-time appointment at your institution is 12 months at 40 hours per week:

- A 9-month part-time appointment has Percent $=9 / 12$, or 0.75 .

- An appointment at 30 hours per week has Percent $=30 / 40$, also 0.75 .

○ An appointment at 30 hours and 9 months has Percent $=0.75 \times 0.75=0.56$.

Enter Percent with two decimal points.

\footnotetext{
21 Dupont Circle NW, Suite 800

Washington, DC 20036

2022962296 telephone

2028720884 fax

http://www.arl.org
} 
- Report salaries for both full-time and part-time professional positions. Salaries for parttime positions should NOT be converted to their full-time equivalents. Report the actual parttime salary paid and indicate the percent appointment for that employee in the appropriate column.

- Include salaries for all professional positions, regardless of whether the salaries come from regular library budget funds or from special funds such as research grants. Please include all professionals involved in the provision of library services, including contract-supported positions.

- The salary figures should be straight gross salary figures. Do not include fringe benefits.

- Provide explanatory footnotes to the reported figures, when necessary, at the end of Part I. Footnotes will be included in the published survey, where appropriate.

- After all data have been entered, make a backup copy of the complete file for your institution's master file. Your backup should include individual names/ID numbers. NOTE: The data submitted to ARL should NOT include individual names/ID numbers, so ARL will NOT be able to supply a copy of your institution's complete file next year.

- Please return the questionnaire the ARL Statistics and Assessment Program by October 1, 2010. Be sure to keep a complete copy of your return, including the electronic version of the data for your files.

\section{INSTRUCTIONS}

\section{Part I: Summary Data (Microsoft Word Form)}

1. Part I of this survey deals with general information for the current fiscal year, 2010-11.

2. Include the Beginning Professional Salary for Law and Medical libraries if included in the survey.

3. The Beginning Professional Salary is the salary that would be paid to a newly hired professional without experience, not necessarily the lowest professional salary paid. In reporting the beginning salary, please use a figure that is actually used or likely to be used for entry-level librarians hired by your library, even if it is your practice rarely to hire entry-level professionals without experience.

4. Please report the 2010-11 Beginning Professional Salary to the best of your knowledge as it exists on July 1, 2010. Do not delay returning your survey with the expectation that more information will be available later.

5. The 2010-11 Average and Median Salary figures will be calculated by ARL from the individual data supplied. 
6. Be sure to fill in the name of the reporting library and the name of the person who prepares the report.

\section{Part II: Individual Data (Microsoft Excel Form)}

1. Part II of this survey requests information on salary, sex, minority status, rank, and years of experience for all filled positions for fiscal year 2010-11. The survey requests information for individuals; aggregate data for each institution will be generated by computer. Vacant positions should be excluded from your report.

2. Data for the Main, Law, and Medical libraries should be reported on separate Excel files.

3. Obtain the Excel file. These instructions assume that you have Microsoft Excel available for use. If not, or if you have trouble opening the files in Excel, please call the ARL Statistics and Assessment Program at (202) 296-2296 or email stats@arl.org.

4. The template Excel file is available at:

http://www.arl.org/stats/annualsurveys/salary/salform10.shtml. This is a generic, blank file that can hold data for Main, Law, or Medical libraries. The file's name is "sal10xxxx.xls"; open the file and save it to your own computer by choosing "Save As" under the File menu. When saving the file, utilize ARL as the prefix for main library reports, use 10 to designate the year (2010-11), and change the " $x x x x$ " in its name to your ARL institution code number, e.g., "ARL101150.xls." Note: use MED for medical libraries, e.g. "MED101150" and LAW to denote law libraries, e.g., "LAW101150."

The file contains columns labeled as follows:

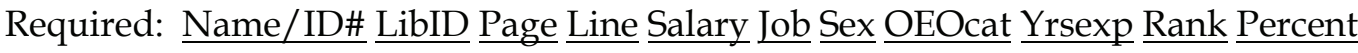
Optional: YrBirth JobAdd LibDeg OthDeg YrsLib

Optional: Hisp NatAm Asian Black $\underline{\text { HawPI White }}$

In the LibID column, enter your ARL Library Institution Code. (See http://www.arl.org/stats/annualsurveys/surveycoord/instno_inam.shtml if you do not know your code.) If you leave this column blank we will fill it in for you when we receive the data.

Columns labeled "Page," "Line," and "Percent" are already filled for you. The numbers in the "Page" and "Line" columns will be used to identify these positions in case of data errors; do not change them. Eleven "pages" of 25 lines each have been provided; if this is not sufficient to list all positions at your institution, copy and paste lines 1-25 of the last page as needed.

\section{Entering Data for Part II: Individual Data (Microsoft Excel Form)}

1. The "Name/ID\#" column is for your internal use, to enter and verify information for staff members by name. ARL does not require that you submit the information in this field to ARL. Please delete this column before sending the file to ARL. Upon receiving this file, ARL will delete any data in this column if you have not deleted them already.

2. The "LibID" will hold your institution's ARL number, for identification purposes. If you do not know your ARL number, you can find it on the Web under ARL Library Institution Codes. If you leave this column blank, it will be filled in by ARL staff. 
3. "Salary" should be entered as it existed on July 1, 2010. Please do not hold up the reporting process for later salary adjustments. Include all filled positions and exclude all vacant positions. Report the actual salary paid. Do not adjust part-time salaries to their full-time equivalents; ARL will do this during the data analysis and verification stage. Do not include fringe benefits.

4. Each position can have only one "Job" code, to be taken from the following list:

DIRLIB Director of Libraries (includes Dean of Libraries and equivalent titles)

ASCDIR Associate Director

ASTDIR Assistant Director

HDMED Head, Medical Library (Human Medicine only)

HDLAW Head, Law Library

HDBR Head, Other Branch Library (including Veterinary Medicine)

FSPEC

Functional Specialist

ARCH Archivists/Curators

BUSI Budget/Fiscal/Business Manager/Facilities

HUMRES Human Resources/Training/Staff Development

ITS Information Technology Systems

ITW Information Technology Web Development

ITP Information Technology Programming/Application Development

MEDIA Media/Multimedia Specialists (including graphics)

PRES Preservation/Conservation

SSPEC Subject Specialist

HDACQ Head, Acquisitions Department

HDCAT Head, Catalog Department/Unit

HDCIRC Head, Circulation

HDCOMP Head, Library and Computer Systems

HDDOC Head, Documents Department

HDMAP Head, Map Room/Department

HDRBM Head, Rare Book/Manuscripts Department

HDREF Head, Reference Department

HDSER Head, Serials Department

HDOTH Head, Other Department/Service/Agency

CAT Catalogers, both general and specialized

REF Reference librarians, both general and specialized

PUBS Public Services, non-supervisory, except reference librarians

TECH Technical Services, non-supervisory, except catalogers

ADMIN Administrative and other units, non-supervisory position

The position categories used in this survey are intended to correspond roughly with the activities carried on in libraries, not with any particular pattern of staff organization or nomenclature. Please use these categories in the manner you feel best applies to your library. If any individual has responsibilities described by more than one of the above categories, choose the category that is most typical of his/her general duties.

Associate or Assistant Director, and Head, Other Branch. Use these codes for all persons at these levels regardless of the area of specialty. If an assistant or associate director is also head of 
a department, choose the category that most reflects the general duties of the person currently in the position.

Specialists. These are of two kinds: Subject Specialists primarily build collections, but may also offer specialized reference and bibliographic services; Functional Specialists are media specialists or experts in management fields such as personnel, fiscal matters, systems, preservation, etc. Specialists may not be, strictly speaking, professional librarians (i.e., have an MLS). The "specialist" category would generally not be used for someone with significant supervisory responsibilities, who should instead be listed as a department head or assistant director (see also note under Assistant Department Head, below).

Functional Specialist sub-codes. Starting with the 2004-05 Salary Survey, the ARL Statistics and Assessment Committee adopted a proposal from the ACRL Personnel Administrators and Staff Development Officers Discussion Group to break down the Functional Specialist category. For each position which would have been labeled FSPEC prior to 2004-05, instead please use one of the eight sub-codes (ARCH, BUSI, HUMRES, ITS, ITW, ITP, MEDIA, PRES) to describe that position. If you cannot determine which sub-code to use, please use the FSPEC code.

Department Heads. Department Heads not specifically included in the above list should be included under the category "Head, Other Department/Service/Agency." Head, Catalog Department should be used either for the department that handles all cataloging, or for the head of a specialized cataloging unit (e.g. copy cataloging or foreign languages). List the head of library automation and computer systems, applications, programming, etc. as HDCOMP unless that person is also an Associate or Assistant Director, in which case use the appropriate administrative code. If there is an intermediate level of management between an Associate or Assistant Director and the professionals who actually carry out the analysis, programming, etc., use HDCOMP to define that intermediate level. Professionals who carry out analysis, programming, etc., should be listed as functional specialists (FSPEC).

Head, Acquisitions Department. Use HDACQ for all of the following positions: (a) head of a department that is responsible for the selection of material (or management of selection activities carried out on a basis encompassing more than a single organizational unit), but not responsible for the placement of orders, payment of invoices, etc.; (b) head of a department responsible for the placement of orders, maintaining on-order files, payment of invoices, etc., but not responsible for selection decisions; (c) head of a department responsible for both the selection decisions (or coordination of selection activities) and for acquiring the material. Libraries that split these two functions between two departments should report more than one professional with the position HDACQ.

Special note concerning Assistant Department Heads. Assistant Department Heads who are responsible for major units and spend the bulk of their time in supervision and revision of the work of others should also be listed as "Head, Other Department/Service/Agency." See additional subcodes below for Head, Cataloging, and Head, Other Department. However, Assistant Head positions responsible for small units or for supervision only in the absence of the head should be reported as non-supervisory or specialist positions as appropriate.]

Administrative. Please note that ADMIN is not only for Administrative Services and related positions, but also can be applied to Public Relations/Communications, 
Development/Fundraising, and all other administrative and/or professional positions which do not have a logical home elsewhere.

5.Please indicate "Sex" with the letter M or F, indicating male or female, respectively.

6. "OEOCat" minority status code, for U.S. university libraries only, should be indicated with one of the following code numbers. (Leave blank if a Canadian library):

$$
\begin{aligned}
& 1=\text { Black } \\
& 2=\text { Hispanic } \\
& 3=\text { Asian or Pacific Islander } \\
& 4=\text { American Indian or Native Alaskan } \\
& 5=\text { Caucasian/Other }
\end{aligned}
$$

7. "YrsExp," or total years of professional experience. For most professional staff members this will mean counting the years since the MLS degree was awarded. When counting, do not subtract interim periods when an individual was not engaged in professional library employment if these periods are short in relation to the overall professional career. Count an academic year contract period as a full year. Be sure to include professional experience in previous positions and in other institutions. The figure should be rounded off to the nearest whole number; for example, a position with 14.5 years of experience would appear as 15 .

\section{Indicate "Rank" using the following system of codes:}

0 The library director. Some systems also use 0 for assistant and/or associate directors.

1 Lowest level in the rank structure, such as an entry-level position.

2-8 Successively higher levels; for example, 5 indicates a higher rank than 2.

9 Rank cannot be determined, or, the individual is outside the organization's rank structure.

Responses concerning rank should be limited to professional librarians, and other professionals who occupy the same ranks as librarians. Leave the rank column blank for professionals who do not occupy these ranks or if the column is not applicable. For example, if the Library Business Officer holds a rank typically used for university administrators but not for librarians, do not supply a rank code for that individual, even if you have included salary and other data.

If multiple ranking structures are used for librarians and these structures are substantially different and not equivalent, enter individual rank information only for that group which represents the largest fraction of "rank-and-file" librarians.

The maximum number of ranks reported here should not exceed the maximum number of ranklevels reported in Part I for individual data under Rank structure. When counting the total number of rank levels, include ranks that may be unoccupied at the present time due to circumstances like unusually high turnover, hiring freezes, etc.

9. "Percent" is used to determine if an employee works full-time or part-time. All full-time employees have Percent $=1.00$, i.e., they work $100 \%$ of a full-time schedule. If Percent is less than 1.00, then the employee works that fraction of a full-time schedule. For example, a $65 \%$ time appointment would be entered as 0.65 . Calculate the percent appointment by dividing the amount of time an employee works by the amount considered to be the norm for full-time 
employment at your institution. For example, if a full-time appointment at your institution is 12 months at 40 hours per week:

o A 9-month part-time appointment has Percent $=9 / 12$, or 0.75 .

o An appointment at 30 hours per week has Percent $=30 / 40$, also 0.75 .

$\circ$ An appointment at 30 hours and 9 months has Percent $=0.75 \times 0.75=0.56$.

Enter Percent with two decimal points.

Optional Questions: (Shown on printed forms as the last 11 columns)

1. Year of Birth (YrBirth). For each individual, record the four-digit year of birth.

2. Position Code Addenda (JobAdd). Use this column to provide additional information only for the following position categories:

a. Associate and Assistant Directors (ASCDIR and ASTDIR). For each category, indicate if the person has a defined area of responsibility using the codes below. Use the code that most closely reflects the general duties of the person in the position.

$\begin{array}{ll}\text { Administrative Services } & \text { ADM } \\ \text { Collection Development } & \text { CDV } \\ \text { Other (or unspecified) } & \text { OTH } \\ \text { Public Services } & \text { PBS } \\ \text { Systems/Automation } & \text { SYS } \\ \text { Technical Services } & \text { TS }\end{array}$

b. Head, Other Branch Library (HDBR). Use the codes below to indicate the subject area of the branch:

$\begin{array}{ll}\text { Humanities/Fine Arts } & \text { HFA } \\ \text { Other } & \text { OTH } \\ \text { Science \& Tech. } & \text { SCI } \\ \text { Social/Behavioral Sci. } & \text { SBS } \\ \text { Undergraduate Library } & \text { UGL }\end{array}$

c. Functional Specialists (FSPEC). Indicate any non-supervisory staff who are primarily responsible for the following activities using the codes below:

$\begin{array}{ll}\text { Acquisitions } & \text { ACQ } \\ \text { Development Officer } & \text { DVP } \\ \text { Interlibrary Loan } & \text { ILL } \\ \text { Marketing/Communications } & \text { COM } \\ \text { Serials } & \text { SER }\end{array}$

d. Subject Specialists, Reference Librarians, Catalogers, and Public Services (SSPEC, REF, CAT, PUBS). Use these codes to indicate non-supervisory main and/or branch library staff who specialize in one of the following subject areas (either a sub-field, or the entire area). Do not add codes for staff in these positions who have broader, other, or mixed subject responsibilities (e.g. more than one field specialty); but do include subject specialists who also have some (i.e. $50 \%$ or less) general or other assignments.

$\begin{array}{ll}\text { Humanities/Fine Arts } & \text { HFA } \\ \text { Science \& Tech. } & \text { SCI }\end{array}$


e. Head, Cataloging Department/Unit (HDCAT). Use the codes below to indicate whether the person is the head of the entire cataloging department for the library, or the head of a specialized unit. If the person is head of the whole department, repeat the same HDCAT code as in the earlier column.

$\begin{array}{ll}\text { Head, all cataloging } & \text { HDCAT } \\ \text { Head, copy cataloging } & \text { HDC } \\ \text { Head, foreign languages } & \text { HDF } \\ \text { Head, non-book formats } & \text { HDN } \\ \text { Head, serials cat. } & \text { HDS } \\ \text { Head, other special cat. } & \text { HDO }\end{array}$

f. Head, Other Department/Service/Agency (HDOTH). For heads of departments not given a separate category in the major list, please add one of the following codes:

$\begin{array}{ll}\text { Audio Visual/Media } & \text { AVM } \\ \text { Archivist } & \text { ARC } \\ \text { Business/Personnel Office } & \text { BPO } \\ \text { Other } & \text { OTH } \\ \text { Interlibrary Loan } & \text { ILL } \\ \text { Preservation } & \text { PRS }\end{array}$

3. Library degrees earned (LibDeg). Use the following codes to indicate the highest academic degree earned in the field of librarianship:

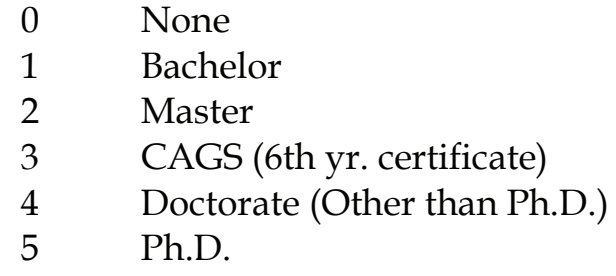

4. Other degrees earned (OtherDeg). Use the following codes to indicate the highest degree earned in fields other than librarianship, including basic undergraduate education:

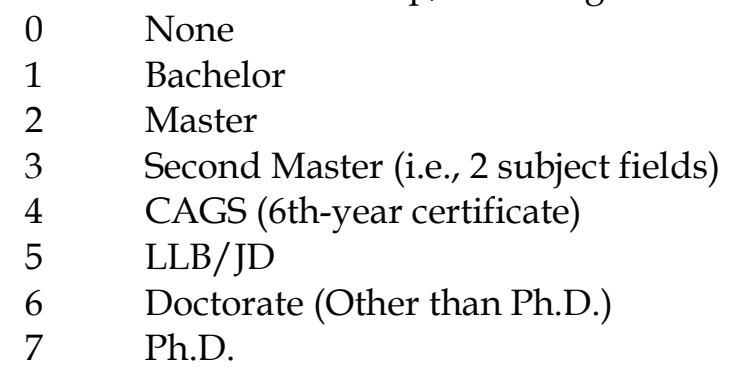

5. Years of professional experience at reporting institution (YrsLib). Use this line to report the number of years of professional experience each librarian has had at your institution. This figure should not exceed the number reported as Total Years of Professional Experience on the main part of the form. 
6. Please complete as much of this section as possible (US libraries only), but do not hold up the reporting process if some of the data requested are not available. Canadian libraries should leave these columns blank. The major change in the revised standard for the classification of federal data on race and ethnicity is that now respondents are able to report more than one race by choosing multiple responses to the following questions

Race and Ethnicity: The U.S. Office of Management and Budget has revised the Standards for the Classification of Federal Data on Race and Ethnicity and according to the new standard there will be five minimum categories for data on race (American Indian or Alaska Native, Asian, Black or African American, Native Hawaiian or Other Pacific Islander, and White) and one category for data on ethnicity ("Hispanic or Latino"). Respondents will be able to report more than one race by choosing multiple responses to the race question. The purpose of the revised classification is to reflect the increasing diversity of the U.S. population that has resulted primarily from growth in immigration and in interracial marriages. The new standards were used by the Bureau of the Census in the 2000 decennial census. ${ }^{1}$ In light of these developments, we are collecting the new classification on race and ethnicity in the ARL Annual Salary Survey on an optional basis.

Ethnicity should be indicated by coding 1 to indicate if the person is of Hispanic or Latino ethnicity, and coding 0 otherwise. The definition of Hispanic or Latino ethnicity is: A person of Cuban, Mexican, Puerto Rican, Cuban, South or Central American, or other Spanish culture or origin, regardless of race.

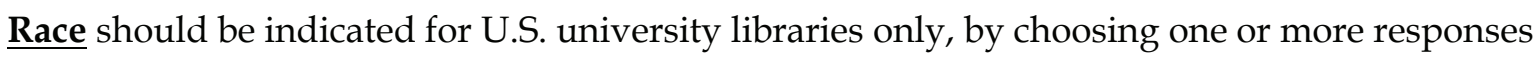
among the five racial categories provided here; $1=$ yes and $0=$ no. You can select multiple racial categories for a person. The definitions of the five racial categories, listed with their respective column names, are:

American Indian or Alaska Native (NatAm): A person having origins in any of the original peoples of North and South America (including Central America) who maintains tribal affiliation or community attachment.

Asian (Asian): A person having origins in any of the original peoples of the Far East, Southeast Asia, or the Indian subcontinent including, for example, Cambodia, China, India, Japan, Korea, Malaysia, Pakistan, the Philippine Islands, Thailand, and Vietnam.

Black or African American (Black): A person having origins in any of the black racial groups of Africa.

Native Hawaiian or Other Pacific Islander (HawPI): A person having origins in any of the original peoples of Hawaii, Guam, Samoa, or other Pacific Islands.

White (White): A person having origins in any of the original peoples of Europe, the Middle East, or North Africa.

1 http://www.census.gov/population/www/socdemo/race/racefactcb.html 
ARL is using the online services of FormStack to collect the data. As part of its privacy policy, FormStack pledges not to sell any collected information to third parties. For the complete FormStack privacy policy, visit http://www.formstack.com/ privacy.html. ARL also accepts Part I and Part II of the salary survey by e-mail from those users who may be uncomfortable submitting the files in FormStack:

- University Libraries: http://www.formstack.com/forms/?987723-c6BynijupA

Be sure to have the electronic copy of your completed salary survey Excel file handy as you will be submitting this file via the FormStack Web form. In addition to the completed Excel file, be prepared to provide the following information as well:

- The name, title, email and phone number of the person who prepared the Excel file. The name, title, email and phone number of your institution's contact person for the salary survey (if different from the person who prepared the Excel file)

- Indicate whether you are submitting salary information for one or more of the following: Main, Law, or Medical library, and the beginning professional salary and rank structure for each.

- For professional salary list the salary that would be paid to a newly hired professional without experience (even if local practice discourages hiring entry-level professionals without experience). Please report the 2010-11 beginning professional salary to the best of your knowledge as it existed on July 1, 2010.

- For rank structure, list the number of unique levels in your institution's rank structure. If you have no levels in your rank structure, use 1 . The number reported here should be equal to the highest number in the "Rank" column of your Excel file (i.e., the number of levels reported in your Excel file should equal the number of levels reported here).

- The names of the libraries that are included and excluded in your figures for the 'general libraries' (these can be main campus libraries or branch campus libraries), as well as any other explanatory information, should be indicated in a footnote. In your footnotes, report any information that would clarify the figures submitted: the inclusion and exclusion of branch campus libraries, a reporting date that is sooner/later than July 1 , 2010, etc. Please make an effort to word your footnotes in a manner consistent with notes appearing in the published report, so that ARL can interpret your footnotes correctly.

Please return the completed questionnaire to the

ARL Statistics and Assessment Program by October 1, 2010.

For assistance, contact Martha Kyrillidou (martha@arl.org), Shaneka Morris (shaneka@arl.org), Gary Roebuck (gary@arl.org) or David Green (david@arl.org).

Tel: 202-296-2296 or Fax: 202-872-0884.

http://www.arl.org/stats/annualsurveys/salary/ 


\section{ARL Annual Salary Survey 2010-11 UNIVERSITY LIBRARY QUESTIONNAIRE}

Note: This is a copy of the form that you will submit electronically at: http://www.formstack.com/forms/?987723-c6BynijupA

\section{Part I: Summary Data}

Reporting Institution Date Returned to ARL

Report Prepared by (name)

Title

Email address Phone number

Contact person (if different)

Title

Email address Phone number

(Note: ARL will calculate the 2010-11 median and average professional salaries for your library from the individual data you supply in Part II (Excel form) of this questionnaire.)

1. Beginning Professional Salary

Main

Law

Health

Beginning professional salary for 2010-11

(Note: The Information shown below must be completed for all three branches (i.e. Main, Law and Health Science Libraries) in Partlof the online form).

\section{Rank Structure.}

Indicate the number of levels in your institution's rank structure for professional librarians. You should report here the maximum number of rank levels, reported in Part II for individual data, under the Rank column.

1 level (i.e., no differentiated levels)

2 levels

3 levels

4 levels

5 levels

more than 5 levels (please specify the number of levels: 


\section{FOOTNOTES}

3a. Please list which libraries are included in the data submitted for the "general" libraries. These can be main campus libraries or branch campus libraries.

3b. Please list which libraries are NOT included in the data submitted for the "general" libraries. These can be main campus libraries or branch campus libraries.

Please indicate any other explanatory information in footnotes. These additional footnotes, if necessary, should be placed in the space below or on attached pages.

Please submit the completed questionnaire to the web form at: http://www.formstack.com/forms/?987723-c6BynijupA

by October 1, 2010.

For assistance, contact Martha Kyrillidou (martha@arl.org), Shaneka Morris (shaneka@arl.org), Gary Roebuck (gary@arl.org) or David Green (david@arl.org).

Tel: 202-296-2296 or Fax: 202-872-0884 


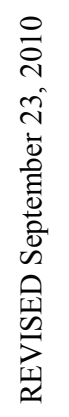

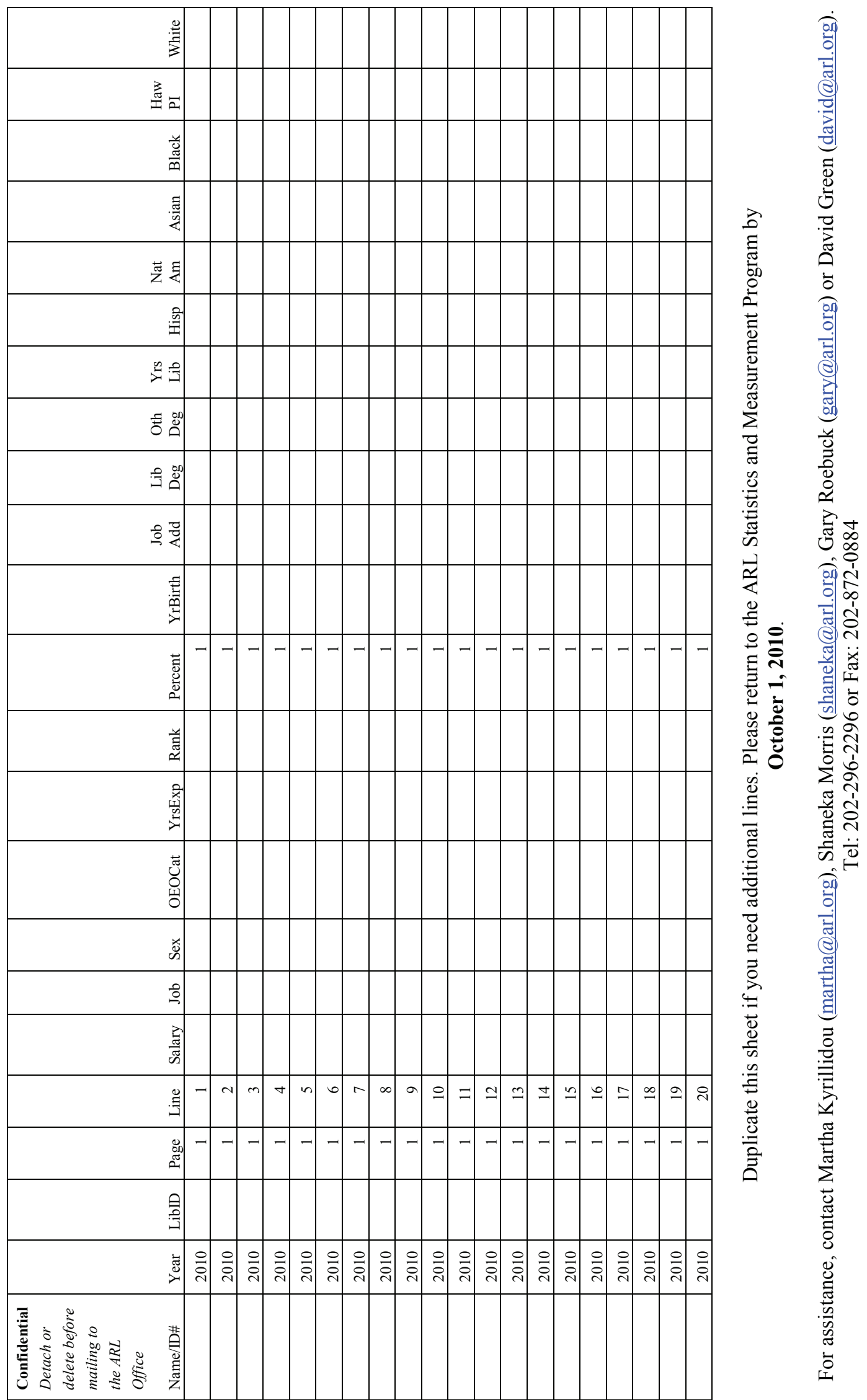


NonUniversity Library QUeSTIONNAIRE AND INSTRUCTIONS 


\title{
ASSOCIATION OF RESEARCH LIBRARIES
}

\author{
ARL ANNUAL SALARY SURVEY 2010-11 \\ NONUNIVERSITY LIBRARY QUESTIONNAIRE
}

\section{General Instructions for Completing the Questionnaire}

1. This survey is concerned with the salaries of professional positions only. Since the criteria for determining professional status vary among libraries, there is no attempt to define the term "professional." Each library should report the salaries of those staff members it considers professionals, irrespective of membership in a collective bargaining unit, and including, when appropriate, staff who are not librarians in the strict sense of the term, such as systems analysts, budget officers, etc.

2. Obtain the Word file. These instructions assume that you have Microsoft Word available for use. If not, or if you have trouble opening the files in Word, please call the ARL Statistics and Assessment Program at (202) 296-2296 or email stats@arl.org.

3. The template Word file is available at: http://www.arl.org/stats/annualsurveys/salary/salform10.shtml. This is a generic, blank form that can hold your data. The file's name is "sal10_nuform.doc"; open the file and save it to your own computer by choosing "Save As" under the File menu. When saving the file, utilize ARL as the prefix, use 10 to designate the year (2010-11), and change the "xxxx" in its name to your ARL institution code number, e.g., "ARL109975.doc."

4. Salaries should be reported for all filled positions. Vacant positions should be excluded from your report.

5. Report 2010-11 salaries as they exist on July 1, 2010. If the library normally increases salaries at a date after July 1 , and the salary as of that later date is known or can be estimated (within $\$ 100$ or so) by the time the questionnaire is due to be returned, please use the higher salary and footnote the effective date and/or whether the reported figures are known or estimated. Please do not hold up the reporting process for later salary adjustments.

6. The Median Salary is the salary that has an equal number of salaries above it and below it. In those libraries with an even number of positions, the median salary is the average of the two salaries that have an equal number of salaries above and below them.

7. The Beginning Professional Salary is the salary that would be paid to a professional without experience, not necessarily the lowest professional salary paid. In reporting the beginning salary, please use a figure that is actually used or likely to be used for entry-level librarians hired by your library. 


\section{ASSOCIATION OF RESEARCH LIBRARIES}

8. Salaries should be reported for both full-time and part-time professional positions. However, salaries for part-time positions should be converted to their full-time equivalents before reporting; do not report the actual part-time salary paid.

9. Salaries should normally be reported on a 12-month basis. If an appointment is for 9 or 10 months at the option of the employee, the actual salary paid should be increased to its 12 -month equivalent. However, if appointments of less than 12 months are required by the employer, report the actual salary paid.

10. The salaries for all professional positions should be included, regardless of whether the salaries come from regular library budget funds or from special funds such as research grants.

11. The salary figures should be straight gross salary figures. Do not include fringe benefits.

12. Explanatory footnotes to the reported figures may be provided when necessary. Footnotes will be included in the published survey.

13. Provide the name of the reporting library and the name of the person who prepares the report.

14. On the second page of the questionnaire (Part II) indicate the number of filled professional positions in each salary range for fiscal years 2009-10 and 2010-11.

\section{Use the newly available Web form for your data submission:}

(http://www.formstack.com/forms/?987727-c6BynijupA). Fill in Part I on the Web and upload your file for Part II through the same interface. NOTE: You must complete the entire submission in a single session. The Web interface does NOT allow you to return and edit your information once it is submitted.

Note: ARL is using the online services of FormStack to collect the data. As part of its privacy policy, FormStack pledges not to sell any collected information to third parties. For the complete FormStack privacy policy, visit http://www.formstack.com/privacy.html. ARL also accepts Part I and Part II of the salary survey by e-mail attachment from those users who may be uncomfortable submitting the files in FormStack.

Please Submit the Web form by October 1, 2010.

For assistance, contact Martha Kyrillidou (martha@arl.org), Shaneka Morris (shaneka@arl.org), Gary Roebuck (gary@arl.org) or David Green (david@arl.org).

Tel: 202-296-2296 or Fax: 202-872-0884 


\section{ARL AnNual SAlary SuRVey 2010-11 \\ NONUNIVERSITY LIBRARY QUESTIONNAIRE}

Note: This is a copy of the form that you will submit electronically at: http://www.formstack.com/forms/?987727-c6BynijupA

Part I: Summary Data

Reporting Institution Date Returned to ARL

Report Prepared by (name)

Title

Email address Phone number

Contact person (if different)

Title

Email address Phone number

1. Complete the table on the back of this sheet by indicating the number of filled or temporarily vacant professional positions in each salary range for fiscal years 2009-10 and 2010-11.

2. Median professional salary for fiscal year 2010-11:

3. Beginning professional salary for 2010-11:

4. Footnotes (please compare with footnotes from surveys of previous years)

a. Law Library salaries are included.
Yes
No
We do not have a Law Library.

b. Medical Library salaries are included.

Yes

No

We do not have a Medical Library.

c. Branch libraries not included (please attach an additional sheet if necessary):

5. Other comments (please attach an additional sheet if necessary): 
Part II Salaries:

Indicate the number of filled professional positions in each salary range for fiscal years 2009-10 and 2010-11.

\begin{tabular}{|c|c|c|}
\hline & \multicolumn{2}{|c|}{ Number of Positions } \\
\hline Salary Range & $2009-10$ & 2010-11 \\
\hline More than 300,000 & & \\
\hline $250,000-299,999$ & & \\
\hline $200,000-250,000$ & & \\
\hline $175,000-199,999$ & & \\
\hline $150,000-174,999$ & & \\
\hline $140,000-149,999$ & & \\
\hline $130,000-139,999$ & & \\
\hline $120,000-129,999$ & & \\
\hline $110,000-119,999$ & & \\
\hline $100,000-109,999$ & & \\
\hline $95,000-99,999$ & & \\
\hline $90,000-94,999$ & & \\
\hline $85,000-89,999$ & & \\
\hline $80,000-84,999$ & & \\
\hline $79,000-79,999$ & & \\
\hline $78,000-78,999$ & & \\
\hline $76,000-77,999$ & & \\
\hline $74,000-75,999$ & & \\
\hline $72,000-73,999$ & & \\
\hline $70,000-71,999$ & & \\
\hline $68,000-69,999$ & & \\
\hline $66,000-67,999$ & & \\
\hline $64,000-65,999$ & & \\
\hline $62,000-63,999$ & & \\
\hline $60,000-61,999$ & & \\
\hline $58,000-59,999$ & & \\
\hline $56,000-57,999$ & & \\
\hline $54,000-55,999$ & & \\
\hline $52,000-53,999$ & & \\
\hline $50,000-51,999$ & & \\
\hline $48,000-49,999$ & & \\
\hline $46,000-47,999$ & & \\
\hline $44,000-45,999$ & & \\
\hline $42,000-43,999$ & & \\
\hline $40,000-41,999$ & & \\
\hline $38,000-39,999$ & & \\
\hline $36,000-37,999$ & & \\
\hline $34,000-35,999$ & & \\
\hline less than 34,000 & & \\
\hline Total Number of Positions & & \\
\hline
\end{tabular}

Please submit the completed questionnaire to the web form at:

http://www.formstack.com/forms/?987727-c6BynijupA

by October 1, 2010.

For assistance, contact Martha Kyrillidou (martha@arl.org), Shaneka Morris (shaneka@,arl.org), Gary Roebuck (gary@arl.org) or David Green (david@arl.org).

Tel: 202-296-2296 or Fax: 202-872-0884 



\section{Footnotes to the ARL Annual Salary Survey, 2010-2011}

All data is as of 1 July 2010 unless otherwise noted.

\section{ALABAMA}

Libraries included: Amelia Gayle Gorgas Library, Angelo Bruno Business Library, Eric and Sarah Rodgers Library for Science and Engineering, McLure Education Library, W.S. Hoole Special Collections Library.

\section{ALBERTA}

Libraries included: Bibliographic and Information Technology Services, HT Coutts Education Library, Humanities \& Social Sciences Library, Faculty Saint-Jean Library, Winspear Business Reference Library, Office of Staff Development \& Training, Cameron Library (including Financial Systems \& Analysis, Science \& Technology Library, Information Technology Services and Research and Special Collections Services), Access Services (including Document Delivery and Interlibrary Loans).

\section{ARIZONA}

Libraries included: Main Library, Science-Engineering Library, Fine Arts Library, Special Collections, Center for Creative Photography.

Data for the Medical library includes the Arizona Health Sciences Library (AHSL) in Tucson and AHSL on the Phoenix Biomedical Campus.

\section{ARIZONA STATE}

Libraries included: Tempe Campus Library, Music Library, Architecture Library, Science Library, Downtown Campus, East Campus, West Campus.

\section{AUBURN}

Libraries included: Main Library, Architecture Library \& Vet Med Library.

\section{BOSTON}

Libraries included: Mugar Memorial Library (Main), Library Theology, and the Gotlieb Archival Research Center.

There are eight levels in the Main and Law library's rank structures.

\section{BOSTON COLLEGE}

Libraries included: O'Neill Library (main library), Educational Resource Center, Social Work Library, Theology and Ministry Library, Bapst Art Library, Burns Library.

\section{BRITISH COLUMBIA}

Libraries included: Art+Architecture+Planning; Asian Library; David Lam Management Library; Education Library; Irving K. Barber Learning Centre (IKBLC); Koerner Library (Humanities \& Social Sciences; Borrower Services); Music Library; Okanagan Library; Rare Books and Special Collections; Robson Square (UBC Library at Robson Square); Science and Engineering; University Archives; Xwi7xwa Library (First Nations House of Learning). Note: Technical Services and Systems are included in IKBLC and Woodward.

Libraries not included: Reading Rooms and Affiliated Libraries.

The University of British Columbia is still negotiating the 2010-2011 contract for faculty/librarians. 


\section{BROWN}

Libraries included: John D. Rockefeller Library, John Hay Library, Orwig Music Library, Sciences Library, List Art Center, John Carter Brown Library.

\section{CALGARY}

Libraries included: MacKimmie Library, Gallagher Library of Geology \& Geophysics; Business Library; Health Information Network Knowledge Centres; Military Museum Library \& Archives; Doucette Library of Teaching Resources (Education Library).

\section{CALIFORNIA, BERKELEY}

Libraries included: Doe, Moffitt, Bancroft, Anthropology, Art History/Classics, Astronomy-Mathematics-Statistics, Bioscience \& Natural Resources, Business \& Economics, Chemistry, C.V. Starr East Asian Library (including Center for Chinese Studies), Earth Sciences, Education-Psychology, Engineering, Environmental Design, Music, Optometry, Physics, Public Health (including Health Sciences Information Services \& Occupational \& Environmental Health), Social Welfare, \& the Northern Regional Library Facility.

Libraries not included: Architecture Visual Resources/CED Visual Resource Center, Continuing Education of the Bar, Earthquake Engineering Research Center, Environmental Design Archives, Ethnic Studies, Giannini Foundation of Agricultural Economics, Institute of Government Studies, Institute for Research on Labor \& Employment, Institute of Transportation Studies, Water Resources Center Archives. Also various departmental libraries: e.g. French, History, Philosophy, Rhetoric, Slavic Languages \& Literature.

Beginning 2004-05, UCB salary figures include administrative stipends, where applicable.

There are six levels in the University of California, Berkeley's rank structure.

\section{CALIFORNIA, DAVIS}

Libraries included: Peter J. Shields Library (Davis Campus); Physical Sciences \& Engineering Library (Davis Campus); Agricultural \& Resource Economics Library (Davis Campus).

Librarians who are department heads have received administrative stipends since July 1, 1999, but the stipends were not included in the salaries reported to ARL until the 2009-10 Salary Survey. We are including those stipends in the department head's salaries (as reported), and we plan to do so in the future.

\section{CALIFORNIA, LOS ANGELES}

Libraries included: The Arts Library, College Library (Undergraduate Library), Eugene and Maxine Rosenfeld Management Library, Music Library, Richard C. Rudolph East Asian Library, Science \& Engineering Library, Social Sciences and Humanities Library (Charles E. Young Research Library), and the Southern Regional Library Facility. Includes data for 12 affiliated libraries on the UCLA campus including the 1) American Indian Studies Center, 2) Ralph M. Bunche African American Studies Center, 3) Asian American Studies Center, 4) Chicano Studies Research Center, 5) Ethnomusicology Archive, 6) Film \& Television Archive, 7) Graduate School of Education \& Information Studies, Department of Information Studies, 8) Institute for Social Science Research, 9) Latin American Center/Hispanic American Periodicals Index, 10) Olive View Medical Center, 11) Grace M. Hunt English Reading Room, and 12) William Andrews Clark Memorial Library.

Librarians who are department heads have received administrative stipends since January 1998; however, these stipends were not included in the salaries reported to ARL prior to 2003. Beginning with the 2003 survey, UCLA now includes those stipends in salaries reported for department heads. Interim department heads also receive stipends and these are reported in the survey.

The General Library Survey includes one Council of Library \& Information Resources (CLIR) Fellow.

Data for Louise M. Darling Biomedical Library includes information for the Pacific Southwest Regional Medical Library, an NLM-funded program that is part of the National Network/Libraries of Medicine based in the Biomedical Library.

108 · ARL Annual Salary Survey 2010-2011 


\section{CALIFORNIA, RIVERSIDE}

Libraries included: Rivera Library: (serving the College of Humanities, Arts and Social Sciences, School of Education, and the School of Business Administration). Orbach Science Library: (serving the College of Natural \& Agricultural Sciences, the College of Engineering, and Biomedical Sciences).

Libraries not included: Media and Music Libraries are not included (there are no librarian employees in these facilities).

\section{CALIFORNIA, SAN DIEGO}

Libraries included: Arts, Social Sciences \& Humanities, Science \& Engineering, International Relations \& Pacific Studies, Scripps Institution of Oceanography, Center for Library \& Instructional Services.

\section{CALIFORNIA, SANTA BARBARA}

Libraries included: The Main and Arts Libraries.

There are eight levels in the University of California, Santa Barbara's rank structure.

\section{CANADA INSTITUTE OF SCIENTIFIC AND TECHNICAL INFORMATION}

Figures for 2009 have not been included. The organization underwent a major restructuring and staff fluctuated considerably during this period.

\section{CASE WESTERN RESERVE}

Libraries included: Kelvin Smith Library, Kulas Music Library, Mandel School of Applied Social Sciences Library and the Harris Library.

\section{CHICAGO}

Libraries included: All libraries including law and medicine, are represented in "Main," we do not differentiate branches, and all librarians are included in the same salary scale and rank structure.

\section{CINCINNATI}

Libraries included: All University of Cincinnati Libraries including the main library, eight college and departmental libraries (Archives and Rare Books; Chemistry-Biology; Classics; Design, Architecture, Art and Planning; Education, Criminal Justice and Human Services; Engineering and Applied Science; Geology-Mathematics-Physics; and Music), and two regional campus libraries (Clermont College and Raymond Walters College).

\section{COLORADO}

Libraries included: Norlin Library (main), Music, Business, Math/Physics, Engineering, Earth Sciences.

The Beginning Professional Salary (BPS) reported for the Law Library is for librarians with an MLS alone. The beginning salary for librarians with an MLS and JD is $\$ 54,000$.

\section{COLUMBIA}

Libraries included: All libraries.

\section{CONNECTICUT}

Libraries included: Homer Babbidge Library, Archives and Special Collections; Music and Dramatic Arts, Pharmacy, Avery Point Campus, Greater Hartford Campus, Stamford Campus, Torrington Campus, Waterbury Campus.

\section{CORNELL}

Libraries included: Africana, Engineering/Physical Sciences, Entomology, Fine Arts, Geneva Experiment Station, Hotel Administration, Management, Mann Library, Math, Music, ILR, Olin/Kroch/Uris, Veterinary Medicine. 


\section{DARTMOUTH}

Libraries included: Baker-Berry Library, Sherman Art Library, Feldberg Business \& Engineering Library, Paddock Music Library, Kresge Physical Sciences Library, Rauner Special Collections Library.

\section{DUKE}

Libraries included: Perkins/Bostock, Lilly, Rare Book, Manuscript and Special Collections, Music, Divinity.

Duke University did not provide a merit pool for Fiscal Year 2011 (1 July 2010 - 30 June 2011). In general, any salary increases are the result in Fiscal Year 2011 of promotion, market adjustment, internal equity adjustment or compression adjustment.

\section{EMORY}

Libraries included: Main, Theology, Business and Oxford College Library.

\section{FLORIDA}

Libraries included: Humanities and Social Sciences, Science, Music, Journalism, Education, Architecture/Fine Arts, Special/Area Studies Collections.

There are six levels in the Main library's rank structure.

\section{FLORIDA STATE}

Libraries included: Main Branch, Engineering and Science.

Libraries not included: Music, Career Center, Art (Ringling), School of Library and Information Studies, Panama City, FL Branch, Panama City, Panama Branch.

We have a six-level rank system: 0- Dean; 1- Assistant Instructor; 2- Associate Instructor; 3- Instruction librarian; 4- Assistant Librarian; 5Associate Librarian; 6- University Librarian.

\section{GEORGE WASHINGTON}

Libraries included: Gelman.

\section{GEORGETOWN}

Libraries included: Bio-ethics.

There are nine levels in the health science library's rank structure.

\section{GEORGIA}

Libraries included: Main Library, Science Library, Map Library, Student Learning Center Library, Curriculum Learning Center Library, several reading rooms and experimental station libraries located throughout the State of Georgia.

The University of Georgia Law Library has nine levels in its rank structure.

\section{GEORGIA TECH}

Libraries included: Main library, Architecture library.

\section{GUELPH}

Libraries included: Main Campus Libraries: McLaughlin Library; Branch Campus Libraries: Ridgetown Campus Library. All salary values were reported in Canadian Dollars (\$CAD). 
Individual rank data have been included only for professional librarians. Rank structure as follows: Library Director assigned rank =0; Assistant Librarian assigned rank =1; Associate Librarian assigned rank = 2; Librarian assigned rank = 3; Non-librarian professionals assigned rank $=9$.

Seven (7) Non-librarian professional positions are now co-funded by the Library budget (0.52 FTE).

\section{HARVARD}

Libraries included: All libraries.

Salary rank structure includes ten levels.

\section{HAWAII}

There are four levels in the Main library's rank structure: rank II to rank V. Rank I only applies to Instructional Faculty not Bibliographic Faculty.

There are four levels in the Law and Health Science library's rank structure: rank II-rank V.

\section{HOUSTON}

The following are included: M.D. Anderson Library, Architecture and Art Library, Music Library, Weston A. Pettey Optometry Library.

The University of Houston Pharmacy Library was closed last year. The collection was incorporated into the MD Anderson Library collection.

\section{HOWARD}

Libraries included: Founders Library, Undergraduate Library; the Architecture, Business, Divinity, and Social Work (branch) Libraries.

Libraries not included: The Moorland Spingarn Research Center.

The library director is an interim director. Professional positions include Associate Librarian, Librarian, Assistant/Associate Director, and Director. In addition, there are several special function positions held by persons with advanced degrees other than the MLS or MLIS.

\section{ILLINOIS, CHICAGO}

Libraries included: Main campus library and Science Library.

Not included are the libraries of the Health Sciences in Chicago, Peoria, Rockford, Urbana.

There are eight levels in the University of Illinois, Chicago's rank system for professional librarians: 8 - Professor, 7 - Associate Professor, 6 - Assistant Professor, 5 - Clinical Professor, 4 - Clinical Associate Professor, 3 - Clinical Assistant Professor, 2 - Instructor, 1 - Academic Professional, 0 - University Librarian (not included in levels according to ARL instructions).

\section{ILLINOIS, URBANA}

Libraries included: All main campus libraries.

\section{INDIANA}

Libraries included: Main campus libraries.

Libraries not included: Dentistry Library; IUPUI University Library; Herron School of Art Library; Columbus Library; and Science and Engineering Library; and other campuses libraries at IU-East, IU-Kokomo, IU-Northwest, IU-Southeast, IU-South Bend, and IPFW-Fort Wayne. 
IOWA

Libraries included: Main and six branches - one campus.

\section{IOWA STATE}

Libraries included: Parks Library (main library) and the Veterinary Medical Library (branch library).

\section{JOHNS HOPKINS}

Libraries included: Sheridan Libraries, Friedheim Library, SAIS Library.

\section{KANSAS}

Libraries included: The main campus Library and all branch libraries.

\section{KENT STATE}

Libraries included: Kent campus: main, architecture, chem/phys, fashion, map, performing arts; Kent State: Ashtabula, East Liverpool, Geauga, Salem, Stark, Trumbull, Tuscarawus.

\section{KENTUCKY}

Libraries included: William T Young Library (Main Campus Library); Agricultural Information Center; Design Library; Education Library; Engineering Library; Equine Library; Fine Arts Library; Science Library; Kentucky Transportation Center.

The Beginning Professional Salary (BPS) reported for the Law Library is for librarians with an MLS alone. The beginning salary for librarians with an MLS and JD is \$52,000.

\section{LAVAL}

There is one library at Laval University (all included).

\section{LOUISVILLE}

Libraries included: Main, Art, Music, University Archives.

\section{MCGILL}

Libraries included: Humanities \& Social Sciences, Islamic Studies, Education, Marvin Duchow Music, Schulich Library of Science and Engineering, Walter Hitschefeld Geographic Information Centre, Macdonald Campus, Howard Ross Library of Management.

McGill librarians ranks are: Tenure or non-tenured as Assistant Librarian, Associate Librarian, and Full Librarian.

\section{MCMASTER}

Libraries included: Mills Memorial Library, H.G. Thode Library, Innis Library.

\section{MANITOBA}

Libraries included: William R Newman Agriculture Library, Architecture and Fine Arts Library, Archives and Special Collections, Elizabeth Dafoe Library, Fr. Harold Drake Library, St. John's College Library, Donald W. Craik Engineering Library, Albert D. Cohen Management Library, Eckhardt-Gramattee Music Library, Sciences and Technology Library, Bill Larson Library, Carolyn Siftono-Helene Fuld Library, Concordia Hospital Library, J.W. Crane Memorial Library, Misercordia Health Centre Library, Riverview Health Centre Virtual Library, Seven Oaks General Hospital Library, Victoria General Hospital Library.

\section{MASSACHUSETTS}

Libraries included: DuBois Library, Science and Engineering Library, Image Collection Library. 


\section{MIAMI}

Libraries included: Main, Music, Architecture, Marine, Business.

\section{MICHIGAN}

Libraries included: Area Programs; Art, Architecture, and Engineering; Asia; Askwith Media; Biological Station; Buhr Remote Shelving; Fine Arts; Foster; Government Documents; Hatcher Graduate; Map; Museums; Music; Papyrology; Shapiro Science; Shapiro Undergraduate; Special Collections.

Libraries not included: Bentley Historical; Clements; Gerald R. Ford Presidential; Kresge Business Administration; Law; Mardigian (Dearborn); Michigan Union; Thompson (Flint); University of Michigan Transportation; Research Institute; Weill Hall.

Figures are as of September 1, 2010. (Law Library)

Beginning salary with MLS only is $\$ 49,000$. With MLS and JD it is $\$ 62,000$. (Law Library)

\section{MICHIGAN STATE}

Libraries included: Main and 5 branch libraries: Veterinary Medicine, Math, Engineering, Business \& Gull Lake/Kellogg Biological Station.

\section{MISSOURI}

Libraries included: Main, Engineering Library, Vet Medicine Library and University Archives.

\section{MIT}

Libraries included: Barker Engineering Library, Science Library, Dewey Library for Management and Social Science, Rotch Library for Architecture and Planning, Humanities Library, Lewis Music Library, Institute Archives and Special Collections, library departments and administration.

There are eight levels in MIT's rank structure.

\section{MONTREAL}

Libraries included: Environmental Development (www.bib.umontreal.ca/AM), Botany (www.bib.umontreal.ca/BV), Chemistry (www. bib.umontreal.ca/CH), Educational Resources (www.bib.umontreal.ca/DI), Education-Communication-Psychology-PsychoeducationBiology (www.bib.umontreal.ca/ED), Geography (www.bib.umontreal.ca/GP), Kinesiology (www.bib.umontreal.ca/SA), Humanities and Social Sciences (www.bib.umontreal.ca/SS), Rare books and Special Collections (www.bib.umontreal.ca/GP), Mathematics and Computer Sciences (www.bib.umontreal.ca/MI), Veterinary (www.bib.umontreal.ca/SA), Music (www.bib.umontreal.ca/MU), Optometry (www.bib. umontreal.ca/SA), Physics (www.bib.umontreal.ca/PY), École polytechnique Library (www.polymtl.ca), HEC Montreal Library (www.hec. ca).

\section{NATIONAL LIBRARY OF MEDICINE}

Data reported by the Federal fiscal year, which runs October 1 to September 30 of each calendar year.

\section{NEBRASKA}

Libraries included: Geology Library, Math Library, Architecture Library, Music Library, Engineering Library.

\section{NEW MEXICO}

Libraries included: Centennial Science \& Engineering Library, Fine Arts \& Design Library, Parish Memorial Library, Zimmerman Library. 
Lecturers (Rank 1) salaries are higher than Assistant Professors (Rank 2) due to years of experience. (Law Library)

\section{NEW YORK}

Libraries included: Elmer Holmes Bobst Library, Institute of Fine Arts, Courant Institute of Mathematical Sciences Library, Institute for the Study of the Ancient World, Jack Brause Midtown Library.

Libraries not included: Bern Dibner Library at the Polytechnic Institute of NYU.

The three ranks are indicated as follows: 1. Library Associate, 2. Assistant Curator, 3. Associate Curator.

\section{NORTH CAROLINA STATE}

Libraries included: (Main) D.H. Hill Library, Design Library, Natural Resources Library, Textiles Library, Veterinary Medicine Library.

\section{NORTHWESTERN}

Libraries included: Main Library, Science \& Engineering Library.

\section{NOTRE DAME}

Libraries included: The Hesburgh Libraries include - Hesburgh Library (Main), Architecture Library, Art Image Library, Business Information Center, Chemistry/Physics Library, Engineering Library, Kellogg/Kroc Information Center, and Mathematics Library.

\section{OHIO}

Libraries included: Main, Music, Depository, Learning Resource Center, Regional campuses (Eastern, Southern, Lancaster, Zanesville, Chillicothe).

The beginning salary applies to main campus hires only. The reference librarian salary that is below the listed beginning professional salary is employed on a branch campus.

\section{OHIO STATE}

Libraries included: Main and branch campus.

Main and Law salaries are as of 10/1/10; Health library salaries are as of 7/1/10.

\section{OKLAHOMA STATE}

Libraries included: OSU-Stillwater (Main, Vet Med, Architecture, Curriculum Materials); OSU-Oklahoma City; OSU-Okmulgee; OSUTulsa.

\section{OREGON}

Libraries included: Main (general) libraries include: Knight Library, Science Library, Architecture and Allied Arts Library, Portland Library and Learning Commons.

\section{OTTAWA}

Libraries included: Main campus Library (Morisset).

\section{PENNSYLVANIA}

Libraries included: University library, Lippincott (business), math/physics (physical sciences), fine arts, veterinary, museum, Center for Advanced Judaic Studies, rare book and manuscript, music.

Libraries not included: Annenberg (communications). 


\section{PENNSYLVANIA STATE}

Libraries included: University Park (main campus), Abington, Altoona, Beaver, Berks, Brandywine, DuBois, Erie, Fayette, Great Valley, Greater Allegheny, Harrisburg, Hazleton, Lehigh Valley, Mont Alto, New Kensington, Shenango, Schuylkill, Wilkes-Barre, WorthingtonScranton, and York..

\section{PITTSBURGH}

Libraries included: University Library System and library directors at regional libraries - Titusville, Johnstown, Bradford, and Greensburg.

Libraries not included: All other staff at regional libraries - Titusville, Johnstown, Bradford, and Greensburg.

\section{PRINCETON}

Libraries included: Firestone Library, East Asian Library, Marquand Library of Art and Archaeology, Lewis Library (Science and Technology Libraries), Engineering Library, Psychology, Mudd Manuscript Library, Cotsen Children's Library, Mendel Music Library, School of Architecture Library and Stokes Library.

\section{PURDUE}

Libraries included: The library system on the West Lafayette campus, consisting of 11 subject-oriented libraries, an undergraduate library, and a special collections research center.

Libraries not included: Libraries at the regional campuses; Purdue North Central (Westville), Purdue Calumet (Hammond) and Indiana University-Purdue University, Fort Wayne.

The four-level rank structure for Purdue Libraries data uses ranks 2 through 4 for Libraries faculty positions: Rank 2 for Assistant Professors, Rank 3 for Associate Professors, and Rank 4 for (Full) Professors. Rank 1 is used for other professional positions; this is a broad category of non-faculty professional positions. These positions include positions that require an MLIS or other advanced degree but are not faculty positions, IT professionals, director of advancement, and other professional staff. Due to market differentials in salaries among various professions and differences in longevity of incumbents, some salaries in Rank 1 are higher than some salaries of Ranks 2 through 4.

\section{QUEEN'S}

Libraries included: Stauffer (Humanities \& Social Science), Douglas (Engineering/Science), Jordan (Special Collections/Music), Education.

\section{ROCHESTER}

Libraries included: River Campus Libraries \& Sibley Music Library.

\section{RUTGERS}

Libraries included: Research and Instructional Services, John Cotton Dana Library and Branches, Paul Robeson Library, Technical and Automated Services.

Libraries not included: Alexander Library, Mabel Smith Douglass Library, Kilmer Library, Library of Science and Medicine and Branches, School of Management and Labor Relations, Center for Alcohol Studies.

\section{SASKATCHEWAN}

Libraries included: Murray Library, Education \& Music Library, Natural Sciences Library, Veterinary Medicine Library, and Engineering Library.

\section{SOUTH CAROLINA}

Libraries included: Thomas Cooper (main) Library, Business Library, Math Library, Music Library, South Caroliniana Library, Moving Image Research Collections. 


\section{SOUTHERN CALIFORNIA}

Libraries included: Main campus libraries.

\section{SUNY-ALBANY}

Libraries included: Main campus and branch libraries.

\section{SUNY-BUFFALO}

Libraries included: The Arts \& Sciences Libraries, Music Library, and Special Collections (University Archives, The Poetry Collection and Rare \& Special Books).

Salary information for classified staff, employees at the SL-2 salary level and temporary hires was not included.

\section{SUNY-STONY BROOK}

Libraries included: Main campus.

\section{SYRACUSE}

Libraries included: Main campus library, Science \& Technology library, Geology \& Math libraries.

Libraries not included: College reading rooms.

\section{TEMPLE}

Libraries included: Paley Library; Science \& Engineering Library; Ambler Campus Library.

Salary for Director for Library Advancement \& External Relations is reported in full, but is split between Libraries and University Advancement (Development) Office.

Minimum beginning professional salary of $\$ 44,004.00$ is based on an 11-month contract. Minimum beginning salary for a 10-month contract is $\$ 40,150$.

\section{TENNESSEE}

Libraries included: Main (University of Tennessee) Library.

Health includes the Memphis Health Sciences Library and Knoxville Hospital Medical Library.

\section{TEXAS}

Figures are as of August 31, 2010.

Libraries included: Dolph Briscoe Center for American History, Harry Ransom Humanities Research Center, and the University of Texas Libraries.

\section{TEXAS A\&M}

Libraries included: Sterling C. Evans Library, Library Annex, Cushing Memorial Library, Medical Sciences Library, Policy Sciences \& Economics Library, West Campus Library.

Libraries not included: Texas A\&M University Library at Qatar, Jack K. Williams Library (Galveston, TX), Technical Resource Center (Architecture Library). 
TEXAS TECH

Figures are as of September 1, 2010.

Libraries included: University Library, Southwest Collections/Special Collections Library, Vietnam Archives.

\section{VANDERBILT}

Libraries included: The Central Library, Divinity Library, Peabody Library, Management Library, Music Library, Science and Engineering Library, Special Collections and University Archives, centralized Technical Services, Library Administration and Television News Archive.

\section{VIRGINIA}

Libraries included: University of Virginia Library, Darden Graduate Business Library, Excludes the John Cook Wyllie Library at the University of Virginia College at Wise.

Libraries not included: University of Virginia College at Wise.

\section{VIRGINIA TECH}

Libraries included: Vet Med Library, Art and Architecture Library.

\section{WASHINGTON}

Libraries included: Libraries on the Seattle, Bothell and Tacoma campuses of the University of Washington.

\section{WASHINGTON STATE}

Libraries included: WSU Pullman, main campus, WSU Spokane, WSU Tri-Cities, WSU Vancouver, WSU Energy Library.

\section{WASHINGTON UNIVERSITY IN ST. LOUIS}

Libraries included: Art \& Architecture, Business, Chemistry, Earth \& Planetary Sciences, East Asian, Music, Physics, Social Work, West Campus.

\section{WATERLOO}

Libraries included: The Dana Porter; Davis Centre; University Map Library and Musagetes Architecture Libraries.

We have a 6-level rank structure.

\section{WAYNE STATE}

Libraries included: The Purdy/Kresge Library, Science and Engineering Library, and Undergraduate Library.

Libraries not included: The Reuther Archives of Labor and Urban Affairs.

\section{WESTERN ONTARIO}

Libraries included: The D.B. Weldon Library, Business Library, Education Library, Music Library, Allyn \& Betty Taylor Library.

Libraries not included: Affiliated College Libraries - King's University College Library, Huron University College Library and Brescia University College Library.

\section{WISCONSIN}

Libraries included: Memorial, Archives, College, Steenbock Agricultural and Life Science, Art, Biology, Business, Chemistry, Geography, Geology, Math, Music, Physics, Social Work, Special collections, Social Science Reading Room, Wendt Engineering. 
YALE

Libraries included: Arts Library (Robert B. Haas Family Arts Library), Bass Library (Anne T. \& Robert M. Bass Library), Beinecke Rare Book and Manuscript Library, Chemistry Library (Sterling Chemistry Library), Classics Library, Divinity School Library, East Asia Library, Engineering and Applied Science Library, Forestry and Environmental Studies Library, Geology Library, Government Documents and Information Center, Lewis Walpole Library, Mathematics Library, Mudd Library, Music Library (Irving S. Gilmore Music Library), Science Library (Kline Science Library), Social Science Library, Sterling Memorial Library.

\section{YORK}

Libraries included: York University Libraries. 


\section{ARL Member Libraries as of January 1, 2011}

The Association of Research Libraries (ARL) represents the interests of 126 libraries that serve major North American research institutions. ARL operates as a forum for the exchange of ideas and as an agent for collective action to influence the forces affecting the ability of these libraries to meet the future needs of scholarship. The ARL Statistics and Measurement program is organized around identifying, collecting, analyzing, and distributing quantifiable information describing the characteristics of research libraries. The program offers publications and special member services, and collaborates with other national and international library statistics programs.

\begin{tabular}{|c|c|c|c|}
\hline Institution & Category & Full Name of Institution & Location \\
\hline Alabama & S & University of Alabama & Tuscaloosa, Alabama \\
\hline Alberta & $\mathrm{C}$ & University of Alberta & Edmonton, Alberta \\
\hline Arizona & S & University of Arizona & Tucson, Arizona \\
\hline Arizona State & $S$ & Arizona State University & Tempe, Arizona \\
\hline Auburn & $S$ & Auburn University & Auburn, Alabama \\
\hline Boston & $\mathrm{P}$ & Boston University & Boston, Massachusetts \\
\hline Boston College & $\mathrm{P}$ & Boston College & Boston, Massachusetts \\
\hline Brigham Young & $\mathrm{P}$ & Brigham Young University & Provo, Utah \\
\hline British Columbia & $\mathrm{C}$ & University of British Columbia & Vancouver, British Columbia \\
\hline Brown & $\mathrm{P}$ & Brown University & Providence, Rhode Island \\
\hline Berkeley, California & S & University of California, Berkeley & California, Berkeley \\
\hline Calgary & $\mathrm{C}$ & University of Calgary & Calgary, Alberta \\
\hline California, Davis & S & University of California, Davis & Davis, California \\
\hline California, Irvine & $\mathrm{S}$ & University of California, Irvine & Irvine, California \\
\hline California, Los Angeles & $\mathrm{S}$ & University of California, Los Angeles & Los Angeles, California \\
\hline California, Riverside & S & University of California, Riverside & Riverside, California \\
\hline California, San Diego & $S$ & University of California, San Diego & La Jolla, California \\
\hline California, Santa Barbara & S & University of California, Santa Barbara & Santa Barbara, California \\
\hline Case Western Reserve & $\mathrm{P}$ & Case Western Reserve University & Cleveland, Ohio \\
\hline Chicago & $\mathrm{P}$ & University of Chicago & Chicago, Illinois \\
\hline Cincinnati & $S$ & University of Cincinnati & Cincinnati, Ohio \\
\hline Colorado & S & University of Colorado & Boulder, Colorado \\
\hline Colorado State & S & Colorado State University & Fort Collins, Colorado \\
\hline Columbia & $\mathrm{P}$ & Columbia University & New York, New York \\
\hline Connecticut & $\mathrm{S}$ & University of Connecticut & Storrs, Connecticut \\
\hline Cornell & $\mathrm{P}$ & Cornell University & Ithaca, New York \\
\hline Dartmouth & $\mathrm{P}$ & Dartmouth College & Hanover, New Hampshire \\
\hline Delaware & $\mathrm{S}$ & University of Delaware & Newark, Delaware \\
\hline Duke & $\mathrm{P}$ & Duke University & Durham, North Carolina \\
\hline Emory & $\mathrm{P}$ & Emory University & Atlanta, Georgia \\
\hline Florida & $\mathrm{S}$ & University of Florida & Gainesville, Florida \\
\hline Florida State & $\mathrm{S}$ & Florida State University & Tallahassee, Florida \\
\hline George Washington & $\mathrm{P}$ & George Washington University & Washington, DC \\
\hline Georgetown & $\mathrm{P}$ & Georgetown University & Washington, DC \\
\hline
\end{tabular}




\begin{tabular}{|c|c|c|c|}
\hline Institution & Category & Full Name of Institution & Location \\
\hline Georgia & $S$ & University of Georgia & Athens, Georgia \\
\hline Georgia Tech & S & Georgia Institute of Technology & Atlanta, Georgia \\
\hline Guelph & $\mathrm{C}$ & University of Guelph & Guelph, Ontario \\
\hline Harvard & $\mathrm{P}$ & Harvard University & Cambridge, Massachusetts \\
\hline Hawaii & $S$ & University of Hawaii & Honolulu, Hawaii \\
\hline Houston & $S$ & University of Houston & Houston, Texas \\
\hline Howard & $\mathrm{P}$ & Howard University & Washington, DC \\
\hline Illinois, Chicago & $S$ & University of Illinois at Chicago & Chicago, Illinois \\
\hline Illinois, Urbana & $S$ & University of Illinois at Urbana & Urbana, Illinois \\
\hline Indiana & S & Indiana University & Bloomington, Indiana \\
\hline Iowa & $S$ & University of Iowa & Iowa City, Iowa \\
\hline Iowa State & $S$ & Iowa State University & Ames, Iowa \\
\hline Johns Hopkins & $\mathrm{P}$ & Johns Hopkins University & Baltimore, Maryland \\
\hline Kansas & S & University of Kansas & Lawrence, Kansas \\
\hline Kent State & S & Kent State University & Kent, Ohio \\
\hline Kentucky & $S$ & University of Kentucky & Lexington, Kentucky \\
\hline Laval & $\mathrm{C}$ & Laval University & Quebec, Quebec \\
\hline Louisiana State & $S$ & Louisiana State University & Baton Rouge, Louisiana \\
\hline Louisville & $\mathrm{S}$ & University of Louisville & Louisville, Kentucky \\
\hline McGill & $\mathrm{C}$ & McGill University & Montreal, Quebec \\
\hline McMaster & $\mathrm{C}$ & McMaster University & Hamilton, Ontario \\
\hline Manitoba & $\mathrm{C}$ & University of Manitoba & Winnipeg, Manitoba \\
\hline Maryland & S & University of Maryland & College Park, Maryland \\
\hline Massachusetts & S & University of Massachusetts & Amherst, Massachusetts \\
\hline MIT & $\mathrm{P}$ & Massachusetts Institute of Technology & Cambridge, Massachusetts \\
\hline Miami & $\mathrm{P}$ & University of Miami & Coral Gables, Florida \\
\hline Michigan & S & University of Michigan & Ann Arbor, Michigan \\
\hline Michigan State & S & Michigan State University & East Lansing, Michigan \\
\hline Minnesota & $S$ & University of Minnesota & Minneapolis, Minnesota \\
\hline Missouri & $S$ & University of Missouri & Columbia, Missouri \\
\hline Montreal & $\mathrm{C}$ & University of Montreal & Montreal, Quebec \\
\hline Nebraska & S & University of Nebraska-Lincoln & Lincoln, Nebraska \\
\hline New Mexico & S & University of New Mexico & Albuquerque, New Mexico \\
\hline New York & $\mathrm{P}$ & New York University & New York, New York \\
\hline North Carolina & S & University of North Carolina & Chapel Hill, North Carolina \\
\hline North Carolina State & $S$ & North Carolina State University & Raleigh, North Carolina \\
\hline Northwestern & $\mathrm{P}$ & Northwestern University & Evanston, Illinois \\
\hline Notre Dame & $\mathrm{P}$ & University of Notre Dame & Notre Dame, Indiana \\
\hline Ohio & S & Ohio University & Athens, Ohio \\
\hline Ohio State & S & Ohio State University & Columbus, Ohio \\
\hline Oklahoma & S & University of Oklahoma & Norman, Oklahoma \\
\hline Oklahoma State & $S$ & Oklahoma State University & Stillwater, Oklahoma \\
\hline
\end{tabular}




\begin{tabular}{|c|c|c|c|}
\hline Institution & Category & Full Name of Institution & Location \\
\hline Oregon & $S$ & University of Oregon & Eugene, Oregon \\
\hline Ottawa & $\mathrm{C}$ & University of Ottawa & Ottawa, Ontario \\
\hline Pennsylvania & $\mathrm{P}$ & University of Pennsylvania & Philadelphia, Pennsylvania \\
\hline Pennsylvania State & $S$ & Pennsylvania State University & University Park, Pennsylvania \\
\hline Pittsburgh & $S$ & University of Pittsburgh & Pittsburgh, Pennsylvania \\
\hline Princeton & $\mathrm{P}$ & Princeton University & Princeton, New Jersey \\
\hline Purdue & S & Purdue University & West Lafayette, Indiana \\
\hline Queen's & $\mathrm{C}$ & Queen's University & Kingston, Ontario \\
\hline Rice & $\mathrm{P}$ & Rice University & Houston, Texas \\
\hline Rochester & $\mathrm{P}$ & University of Rochester & Rochester, New York \\
\hline Rutgers & $S$ & Rutgers University & New Brunswick, New Jersey \\
\hline Saskatchewan & $\mathrm{C}$ & University of Saskatchewan & Saskatoon, Saskatchewan \\
\hline South Carolina & $S$ & University of South Carolina & Columbia, South Carolina \\
\hline Southern California & $\mathrm{P}$ & University of Southern California & Los Angeles, California \\
\hline Southern Illinois & S & Southern Illinois University & Carbondale, Illinois \\
\hline SUNY-Albany & S & University at Albany, State University of New York & Albany, New York \\
\hline SUNY-Buffalo & $S$ & University at Buffalo, State University of New York & Buffalo, New York \\
\hline SUNY-Stony Brook & S & State University of New York at Stony Brook & Stony Brook, New York \\
\hline Syracuse & $\mathrm{P}$ & Syracuse University & Syracuse, New York \\
\hline Temple & S & Temple University & Philadelphia, Pennsylvania \\
\hline Tennessee & S & University of Tennessee & Knoxville, Tennessee \\
\hline Texas & S & University of Texas & Austin, Texas \\
\hline Texas A\&M & S & Texas A\&M University & College Station, Texas \\
\hline Texas Tech & S & Texas Tech University & Lubbock, Texas \\
\hline Toronto & $\mathrm{C}$ & University of Toronto & Toronto, Ontario \\
\hline Tulane & $\mathrm{P}$ & Tulane University & New Orleans, Louisiana \\
\hline Utah & S & University of Utah & Salt Lake City, Utah \\
\hline Vanderbilt & $\mathrm{P}$ & Vanderbilt University & Nashville, Tennessee \\
\hline Virginia & $S$ & University of Virginia & Charlottesville, Virginia \\
\hline Virginia Tech & $S$ & Virginia Polytechnic Institute \& State University & Blacksburg, Virginia \\
\hline Washington & S & University of Washington & Seattle, Washington \\
\hline Washington State & S & Washington State University & Pullman, Washington \\
\hline Washington U.-St. Louis & $\mathrm{P}$ & Washington University & St. Louis, Missouri \\
\hline Waterloo & $\mathrm{C}$ & University of Waterloo & Waterloo, Ontario \\
\hline Wayne State & $S$ & Wayne State University & Detroit, Michigan \\
\hline Western Ontario & $\mathrm{C}$ & University of Western Ontario & London, Ontario \\
\hline Wisconsin & S & University of Wisconsin & Madison, Wisconsin \\
\hline Yale & $\mathrm{P}$ & Yale University & New Haven, Connecticut \\
\hline York & $\mathrm{C}$ & York University & North York, Ontario \\
\hline Boston Public Library & $\mathrm{N}$ & Boston Public Library & Boston, Massachusetts \\
\hline Canada Inst. SciTech Info. & $X$ & Canada Inst. for Scientific \& Technical Information & Ottawa, Ontario \\
\hline
\end{tabular}




\begin{tabular}{lll} 
Institution & Category & Full Name of Institution \\
\hline Center for Research Libs. & N & Center for Research Libraries \\
Lib. \& Archives Canada & X & Library and Archives Canada \\
Library of Congress & N & Library of Congress \\
Natl. Agricultural Lib. & N & National Agricultural Library \\
Natl. Archives \& Records & N & National Archives and Records Administration \\
Natl. Library of Medicine & N & National Library of Medicine \\
New York Public Library & N & New York Public Library \\
New York State Library & N & New York State Library \\
Smithsonian Institution & N & Smithsonian Institution \\
S - US public university & & \\
P - US private university & & \\
C - Canadian university & & \\
N - US nonuniversity & &
\end{tabular}

Ottawa, Ontario

Washington, DC

Beltsville, Maryland

Washington, DC

Bethesda, Maryland

New York, New York

Albany, New York

Washington, DC 